\title{
Activation of the ATR kinase by the RPA-binding protein ETAA1
}

Haahr, Peter; Hoffmann, Saskia; Tollenaere, Maxim A X; Ho, Teresa; Toledo Lazaro, Luis Ignacio; Mann, Matthias; Bekker-Jensen, Simon; Räschle, Markus; Mailand, Niels

Published in:

Nature Cell Biology

DOI:

$10.1038 /$ ncb3422

Publication date:

2016

Document version

Peer reviewed version

Citation for published version (APA):

Haahr, P., Hoffmann, S., Tollenaere, M. A. X., Ho, T., Toledo Lazaro, L. I., Mann, M., Bekker-Jensen, S.,

Räschle, M., \& Mailand, N. (2016). Activation of the ATR kinase by the RPA-binding protein ETAA1. Nature Cell Biology, 18(11), 1196-1207. https://doi.org/10.1038/ncb3422 


\section{Activation of the ATR kinase by the RPA-binding protein ETAA1}

Peter Haahr ${ }^{1}$, Saskia Hoffmann ${ }^{1, *}$, Maxim A. X. Tollenaere ${ }^{1, *}$, Teresa Ho ${ }^{1,2,{ }^{*}}$, Luis

Ignacio Toledo ${ }^{1}$, Matthias Mann ${ }^{3}$, Simon Bekker-Jensen ${ }^{1}$, Markus Räschle ${ }^{3,4, \#}$, Niels Mailand ${ }^{1,2, \#}$

${ }^{1}$ The Novo Nordisk Foundation Center for Protein Research, and ${ }^{2}$ Center for Chromosome Stability, Faculty of Health and Medical Sciences, University of Copenhagen, Blegdamsvej 3B, 2200 Copenhagen, Denmark; ${ }^{3}$ Department of Proteomics and Signal Transduction, Max Planck Institute of Biochemistry, Am Klopferspitz 18, 82152 Martinsried, Germany; ${ }^{4}$ Present address: Department of Molecular Genetics, TU Kaiserslautern, Paul-Ehrlich Strasse 24, 67663 Kaiserslautern, Germany

*Equal contribution. ${ }^{\#}$ Correspondence and requests for materials to M.R. (email: raeschle@biologie.uni-kl.de) and N.M. (e-mail: niels.mailand@cpr.ku.dk) 


\begin{abstract}
Activation of the ATR kinase following perturbations to DNA replication relies on a complex mechanism involving ATR recruitment to RPA-coated singlestranded DNA via its binding partner ATRIP and stimulation of ATR kinase activity by TopBP1. Here, we discovered an independent ATR activation pathway in vertebrates, mediated by the uncharacterized protein ETAA1 (Ewing's tumor-associated antigen 1). Human ETAA1 accumulates at DNA damage sites via dual RPA-binding motifs and promotes replication fork progression and integrity, ATR signaling and cell survival after genotoxic insults. Mechanistically, this requires a conserved domain in ETAA1 that potently and directly stimulates ATR kinase activity independently of TopBP1. Simultaneous loss of ETAA1 and TopBP1 gives rise to synthetic lethality characterized by massive genome instability and abrogation of ATR-dependent signaling. Our findings demonstrate that parallel TopBP1- and ETAA1mediated pathways underlie ATR activation and that their combined action is essential for coping with replication stress.
\end{abstract}




\section{Introduction}

Alterations to the content and organization of cellular genomes, inflicted by numerous genotoxic insults, may lead to pathological conditions such as cancer, premature aging and neurodegeneration ${ }^{1,2}$. To mitigate this threat, cells mount a coordinated DNA damage response (DDR) that promotes repair of DNA lesions and delays cell cycle progression until genome integrity has been restored ${ }^{3,4}$. The phosphatidyl inositol 3' kinase-related kinases ATM and ATR are master organizers of the DDR that trigger damage-specific signaling cascades through direct phosphorylation of large numbers of effector proteins, including the downstream checkpoint kinases Chk1 and Chk $2^{4-10}$. The critical physiological importance of these kinases in protecting genome stability is illustrated by patients with Ataxia telangiectasia and Seckel syndromes, caused by mutations in ATM and ATR, respectively, which manifest with a range of severe disabilities ${ }^{11,12}$. Whereas ATM is mainly activated by DNA double-strand breaks (DSBs), ATR is the major kinase responding to replication stress, a potentially deleterious condition defined by a slowdown or block to replication fork progression, which arises frequently from a broad range of perturbations including DNA damage, nucleotide shortage, and replicationtranscription collisions ${ }^{13,14}$. ATR is essential for ensuring the fidelity of DNA replication after such insults, suppressing new origin firing, preventing fork collapse and promoting the restart of stalled forks. Consequently, disruption of the ATR gene causes early embryonic lethality in mice ${ }^{15,16}$.

Replication Protein A (RPA)-coated single-stranded DNA (ssDNA) regions generated by uncoupling of replicative helicase and polymerase movements upon fork stalling or by DSB end resection provide a main stimulus for activation of ATR, which is 
recruited to these structures via its obligate binding partner ATRIP ${ }^{17-19}$. In parallel, the PCNA-like Rad9-Rad1-Hus1 (9-1-1) complex is loaded onto junctions of ssDNA and double-stranded DNA (dsDNA), leading to recruitment of the multi-BRCT domaincontaining protein TopBP1 via Rad9 phosphorylation, the Mre11-Rad50-Nbs1 (MRN) complex, and the RHINO protein ${ }^{20-25}$. TopBP1 directly stimulates ATR kinase activity by means of an ATR-activating domain (AAD) that may promote conformational changes of the ATR kinase domain ${ }^{26,27}$, and whose integrity is essential for viability in mice ${ }^{28}$.

While TopBP1 is the only protein known to harbor an AAD in higher eukaryotes, two additional proteins, the Rad9 orthologue Ddc1 and the exonuclease Dna2, also contain AADs in the yeast $S$. cerevisiae ${ }^{29,30}$. These factors play partially redundant roles in promoting activation of Mec1, the ATR orthologue in budding yeast, and simultaneous knockout of all three AAD-containing proteins is required to abrogate Mec1-dependent checkpoint signaling in S phase ${ }^{30}$. Whether ATR activation in higher eukaryotes involves AAD-containing proteins other than TopBP1 is therefore an important outstanding question. In this study, we demonstrate that the uncharacterized protein ETAA1 (Ewing's tumor-associated antigen 1) is an RPA-binding protein that harbors a potent $\mathrm{AAD}$ and operates in parallel with, but independent of, the canonical 9-1-1/TopBP1 axis to promote ATR signaling upon replication stress. Loss of ETAA1 disrupts the integrity of the DNA replication programme, sensitizes cells to replication stress and displays synthetic lethality with TopBP1 depletion, characterized by abrogation of ATR-dependent checkpoint signaling and massive genomic instability. Thus, ATR activation in human cells relies on dual TopBP1- and 
ETAA1-mediated pathways whose combined action is essential for coping with replication stress.

\section{Results}

\section{Systematic profiling of DNA double-strand break (DSB)-containing chromatin}

We recently developed a mass spectrometry-based approach (CHROMASS) to systematically profile protein recruitment to chromatin containing DNA interstrand crosslinks in Xenopus egg extracts ${ }^{31}$. To extend these surveys, we applied CHROMASS and label-free mass spectrometry to study protein enrichment dynamics at chromatin modified with nuclease-generated DNA double-strand breaks (DSBs) (Figure 1a). At early time points, we observed strong and near-exclusive enrichment of virtually all known core factors in classical non-homologous end-joining (c-NHEJ), a major DSB repair pathway (Figure 1b,c;Table S1,S2). At later time points, the ssDNA-binding RPA complex (rfa1-3) and known RPA interactors (including ATRATRIP, SMARCAL1 (smal1), rfwd3 and helb) also became enriched on the damaged chromatin (Figure 1b,d;Table S1,S2), indicative of progressive DSB end resection. In addition to many established DSB repair factors, these screens revealed potential new components of DNA damage signaling and repair pathways, one of which we characterize below. These data demonstrate that CHROMASS is a powerful method for comprehensive profiling of the dynamic protein landscape at DSB-modified chromatin.

\section{ETAA1 is a potential DDR factor linked to RPA}


Among factors showing recruitment to DSB-containing chromatin in the Xenopus system, we identified an uncharacterized protein, etaa1, which became markedly enriched on damaged chromatin together with RPA and RPA-binding factors (Figure 1b-d). High sequence coverage allowed robust quantification of etaal across the dataset, revealing strikingly similar intensity profiles of etaa1, RPA subunits and known RPA interactors (Figure S1a-c). Moreover, etaal showed strong enrichment on undamaged chromatin upon inhibition of DNA polymerases by Aphidicolin (Figure S1d;Table S3), which causes extensive RPA-ssDNA generation through uncoupling of replicative polymerases and helicases ${ }^{17}$. This suggests that the function of etaal is tightly linked to RPA.

To characterize the cellular function of etaa1, we focused on the human orthologue, ETAA1 (Ewing's tumor-associated antigen 1), an uncharacterized 926-amino acid protein $^{32}$. When expressed stably in human cells, GFP-tagged ETAA1 underwent prominent redistribution to laser-induced DSBs demarcated by $\gamma \mathrm{H} 2 \mathrm{AX}$ positivity (Figure 1e,f). Consistent with the strong clustering of etaa1 with RPA and RPAinteractors in CHROMASS experiments (Figure S1b), ETAA1 was only recruited to DNA damage sites in cells displaying RPA1 accumulation, and the ETAA1 and RPA1 signals perfectly co-localized in a microfoci-like pattern contained within the more expanded $\gamma \mathrm{H} 2 \mathrm{AX}$ regions at laser stripes (Figure 1e,f) ${ }^{33}$. ETAA1 also formed nuclear foci colocalizing with RPA after short-term exposure to Camptothecin (CPT), which induces replication-dependent DSBs, and Hydroxyurea (HU), which causes helicase-polymerase uncoupling but little DSB formation (Figure 1g). We conclude that ETAA1 is recruited to RPA-coated ssDNA regions generated upon genotoxic insults in vertebrate cells. 


\section{ETAA1 accumulates at DNA damage sites through direct binding to RPA}

The observations above suggested that ETAA1 might be recruited to DNA damage sites through direct binding to RPA. Consistently, inhibition of RPA accumulation at DSB sites by knockdown of CtIP, a key factor in DSB end resection ${ }^{34}$, or RPA1, suppressed ETAA1 recruitment to damaged DNA, while depletion of core homologous recombination (HR) factors such as BRCA2 or RAD51 had no effect (Figure 2a;S2a). Moreover, ETAA1 and RPA subunits interacted firmly in reciprocal co-immunoprecipitation assays (Figure 2b,c). Expression of a series of ETAA1 fragments showed that its $\mathrm{C}$-terminal portion contained the RPA-binding determinant (Figure S2b). Inspection of the primary sequence of this region revealed an evolutionarily conserved motif in the extreme C-terminus of ETAA1 showing striking homology with the RPA2-binding motifs in SMARCAL1, XPA and TIPIN (Figure

$2 \mathrm{~d})^{35-37}$. Indeed, removal of the C-terminal region in RPA2 recognized by these motifs impaired its interaction with ETAA1 (Figure 2c). Deletion of the ETAA1 C-terminus containing the putative RPA2-binding motif $(\triangle \mathrm{RBM} 2)$ or point mutations of key conserved residues predicted to impair its functional integrity ${ }^{35,36}$ suppressed the RPA-binding ability of ETAA1 and its recruitment to DSBs (Figure 2b,e,f;S2c-f). However, the ETAA1 $\triangle$ RBM2 mutant displayed weak accumulation at DNA damage sites in a small proportion of cells (Figure 2e,f), suggesting that additional sequence determinants in ETAA1 also contribute to its DNA damage recruitment. To this end, we noticed a sequence in the central portion of ETAA1 with similarity to known RPA1-binding motifs in ATRIP, NBS1 and RAD9 (Figure 2d) ${ }^{38}$. While deletion of this sequence alone $(\triangle \mathrm{RBM} 1)$ had no impact on the ability of ETAA1 to interact with RPA and DNA damage sites, simultaneous ablation of both RPA-binding motifs $(\triangle \mathrm{RBM} 1+2)$ fully suppressed ETAA1 recruitment to damaged DNA but did not 
impair RPA accumulation (Figure 2b,e,f;S2e,f). These data suggest that ETAA1 contains two RPA-binding determinants that jointly underlie its stable retention at DNA damage sites.

\section{ETAA1 contains an ATR-activating domain}

We next asked how ETAA1 functions in the DDR. Strikingly, overexpression of fulllength ETAA1 led to strong, pan-nuclear $\gamma \mathrm{H} 2 \mathrm{AX}$ positivity independently of its binding to RPA (Figure 3a,b). Instead, deleting N-terminal portions of ETAA1 suppressed its ability to promote $\gamma \mathrm{H} 2 \mathrm{AX}$ formation, and overexpression of these regions alone was sufficient to trigger $\mathrm{H} 2 \mathrm{AX}$ phosphorylation, similar to the TopBP1 ATR-activating domain (AAD) (Figure 3a,b;S3a,b) ${ }^{39}$. Using ETAA1 deletion constructs, we mapped the minimal $\gamma \mathrm{H} 2 \mathrm{AX}$-promoting determinant in ETAA1 to amino acids 56-220, a conserved region harboring a predicted coiled coil domain (Figure 3a-c;S3a,b). For reasons described below, we refer to this region as AAD (ATR-activating domain) (Figure 3c;S3a). In-frame deletion of this sequence ( $\triangle \mathrm{AAD}$ ) had no impact on ETAA1 recruitment to DNA damage sites but abolished $\gamma \mathrm{H} 2 \mathrm{AX}$ formation upon ETAA1 overexpression (Figure 3a,b), further suggesting that this activity is uncoupled from its RPA-binding ability. To characterize the ability of ETAA1-AAD to induce $\gamma \mathrm{H} 2 \mathrm{AX}$ formation, we fused this sequence to GFP and an estrogen receptor variant (ERT2), allowing for swift nuclear translocation of otherwise cytoplasmic GFP-ETAA1-AAD-ERT2 upon treatment with 4-hydroxytamoxifen (4-OHT) (Figure 3c,d). Exposure of cells stably expressing GFP-ETAA1AAD-ERT2 to 4-OHT triggered rapid and robust pan-nuclear $\gamma \mathrm{H} 2 \mathrm{AX}$ positivity irrespective of cell cycle position (Figure 3d-f;S3c). This effect resembled that of an analogous system for 4-OHT-controlled nuclear expression of TopBP1-AAD ${ }^{39}$, 
however ETAA1-AAD promoted $\gamma \mathrm{H} 2 \mathrm{AX}$ formation at lower expression levels than TopBP1-AAD (Figure S3d). Several lines of evidence suggested that similar to TopBP1-AAD ${ }^{39}$, the $\gamma \mathrm{H} 2 \mathrm{AX}$ positivity caused by ETAA1-AAD overexpression was not a consequence of DNA breakage: First, the ETAA1-AAD-induced $\gamma \mathrm{H} 2 \mathrm{AX}$ signal was pan-nuclear rather than focal (Figure 3a,d,e) and it was not accompanied by RPA foci formation and chromatin loading (Figure S3e). Second, treatment with kinase inhibitors or siRNAs showed that the $\gamma \mathrm{H} 2 \mathrm{AX}$-promoting ability of ETAA1-AAD was independent of the DSB-responsive kinases ATM and DNA-PK, whereas the kinase activity of ATR was essential (Figure 3f; see also Figure S6a-c).

Because ATR is capable of phosphorylating H2AX like ATM and DNA-PK ${ }^{40}$, these data suggested that ETAA1-AAD directly stimulates ATR kinase activity similar to TopBP1-AAD. Indeed, both full-length ETAA1 and ETAA1-AAD alone interacted with ATR in co-immunoprecipitation assays (Figure 3g;S3f). When incubated with purified ATR-ATRIP complex, recombinant ETAA1-AAD markedly stimulated ATR kinase activity towards MCM2 in vitro (Figure 3h), strongly suggesting that this domain is a bona fide AAD. The TopBP1-AAD contains a conserved tryptophan residue (W1145 in human TopBP1) that is essential for its ability to stimulate ATR kinase activity ${ }^{26}$. Strikingly, the sequence spanning this residue displayed similarity to a region within ETAA1-AAD, and targeted point mutations of aromatic amino acids in this sequence $\left(\mathrm{AAD}^{m u t}\right.$ ) were sufficient to abrogate $\gamma \mathrm{H} 2 \mathrm{AX}$ formation triggered by overexpression of full-length ETAA1 or its AAD alone (Figure 3a-c;S3a).

Interestingly, ETAA1-AAD contains several potential ATR phosphorylation sites and was phosphorylated by ATR in vitro (Figure 3h;S3a). Moreover, endogenous ETAA1 showed a prominent ATR-dependent mobility shift after replication stress (Figure 
S3g), suggesting that it undergoes phosphorylation by ATR in response to such insults. Finally, mass spectrometry-based analysis of ATR-dependent phosphorylation changes induced by ETAA1-AAD overexpression showed that many ATR phosphorylation sites, including two in ETAA1-AAD itself, were significantly upregulated under these conditions (Figure 3i;S3h,i;Table S4). We confirmed biochemically for H2AX and Chk1 that nuclear overexpression of ETAA1-AAD enhanced their ATR-dependent phosphorylation (Figure 3j). Altogether, these results demonstrate that ETAA1 harbors an AAD capable of directly and potently stimulating ATR kinase activity.

\section{ETAA1 promotes ATR-mediated signaling and cell survival after replication stress}

Prompted by the findings above, we generated human HCT116 cell lines with targeted ETAA1 knockout (ETAA1A) to investigate whether ETAA1 promotes ATR signaling upon genotoxic stress. Indeed, while ablation of ETAA1 expression did not markedly affect overall cell cycle distribution (Figure S4a), ATR-dependent phosphorylation of RPA2 at S33 in response to CPT treatment was strongly diminished in HCT116 ETAA1 $\triangle$ cells (Figure 4a-c). Likewise, RPA2 S4/S8 phosphorylation, which occurs downstream of S33 phosphorylation in CPT-treated cells $^{41}$, was clearly defective in ETAA1 $\triangle$ cells (Figure 4a,b). Similar effects could be seen in HeLa cells ablated for ETAA1 expression (Figure S4b,c). Importantly, ETAA1 knockout did not impair RPA loading at CPT-induced DSBs (Figure 4b-e), suggesting that ETAA1 loss impairs the response to CPT at the level of checkpoint signaling but not ssDNA generation via DSB end resection. In response to HUinduced helicase-polymerase uncoupling, ATR is essential to prevent RPA exhaustion and fork breakage by suppressing new origin firing, and inhibition of ATR in HU- 
treated cells therefore leads to excessive DSB formation and RPA hyperphoshorylation ${ }^{42}$. Consistently, HU-treated ETAA1 $\triangle$ cells showed increased levels of RPA2 phosphorylation similar to the effect of ATR inhibition (Figure S4d $)^{42}$. In contrast, ATR-dependent phosphorylation of Chk1 after treatment with CPT or HU was not markedly altered in ETAA1-deficient cells (Figure 4a;S4c), indicating that loss of ETAA1 differentially affects ATR substrates.

Further supporting a role of ETAA1 in promoting ATR signaling, loss of ETAA1 rendered cells highly sensitive to replication stress induced by HU, CPT or Mitomycin C (MMC), but not to clastogens such as Etoposide that generate DSBs independently of replication (Figure $4 \mathrm{f}-\mathrm{h} ; \mathrm{S} 4 \mathrm{e}-\mathrm{g}$ ). Depletion of ETAA1 by siRNAs phenocopied the effect of ETAA1 knockout in cell survival assays (Figure S4h-j). Importantly, complementation of ETAA1A cells with ectopic ETAA1 wild-type (WT), but not the $\triangle \mathrm{RBM} 1+2, \triangle \mathrm{AAD}$ and $\mathrm{AAD}^{\text {mut }}$ mutants, fully rescued their hypersensitivity to CPT and partially restored survival after HU or MMC (Figure 4f$\mathrm{h} ; \mathrm{S} 4 \mathrm{k}, 1)$. Because ETAA1 is a potent ATR activator, it is possible that expression of stably reconstituted GFP-ETAA1 at levels above that of endogenous ETAA1 (Figure S4k) may adversely affect cellular responses to certain genotoxic agents. We conclude that ETAA1 has an important role in promoting cell survival following replication stress, in a manner that requires both its ATR-activating and RPA-binding abilities.

\section{ETAA1 is required for $\mathrm{DNA}$ replication integrity}

We performed DNA fiber assays to understand how the absence of ETAA1 sensitizes cells to replication stress. By labeling cells with consecutive pulses of 
chlorodeoxyuridine (CldU) and 5'-iododeoxyuridine (IdU), we found that ETAA1 deficiency in both HCT116 and HeLa cells caused a strong reduction in replication fork speed in otherwise unperturbed cells (Figure 5a;S5a). Moreover, ETAA1deficient cells showed marked asymmetry of bidirectional replication forks from individual replication origins (Figure $5 \mathrm{~b} ; \mathrm{S} 5 \mathrm{~b}$ ), suggesting that ETAA1 loss gives rise to a higher incidence of fork pausing and stalling. The diminished fork speed in ETAA1A cells was unexpected, given the similar cell cycle and EdU incorporation profiles of WT and ETAA1A cells (Figure S4a,b). However, we found that in both HCT116 and HeLa ETAA1 $\triangle$ cells, the slower fork progression in ETAA1 $\triangle$ cells was accompanied by a dramatically increased rate of new origin firing (Figure $5 \mathrm{c} ; \mathrm{S} 5 \mathrm{c}$ ). Hence, loss of ETAA1 deregulates DNA replication initiation and elongation, similar to reported effects of ATR inhibition ${ }^{43}$. These defects were fully corrected by stably reconstituted WT ETAA1 (Figure 5a-c). Further supporting a key role of ETAA1 in promoting ATR signaling during replication, we found that the basal $\gamma \mathrm{H} 2 \mathrm{AX}$ level in unstressed cells, which was ATR-dependent and restricted to S/G2 phase cells, was also prominently reduced in ETAA1 $\triangle$ cells, whereas depletion of TopBP1 had no effect (Figure 5d;S5d-g).

We next assessed how ETAA1 loss affects DNA replication status after fork-stalling insults. When cells were pulse-labeled with CldU and then with IdU in the presence of CPT, ETAA1 was again important for suppressing fork progression defects and new origin firing, in an AAD-dependent manner (Figure 5e,f). In separate experiments, we found that ETAA1 $\triangle$ cells subjected to prolonged low-dose treatment with the DNA polymerase inhibitor Aphidicolin showed markedly elevated levels of abnormal nuclei and micronuclei as well as 53BP1 nuclear bodies in G1 phase ${ }^{44}$ 
(Figure 5g,h), further demonstrating a compromised ability of these cells to respond to replication problems. These data show that like ATR, ETAA1 is important for the integrity of DNA replication during both unperturbed and stressful conditions.

\section{ETAA1 and TopBP1 function in independent but parallel ATR activation pathways}

Because both ETAA1 and TopBP1 contain AADs, we reasoned that they might cooperatively promote ATR-dependent signaling after replication stress. We therefore asked whether they function in joint or separate ATR activation pathways. Knockdown of TopBP1 or the 9-1-1 subunit Rad9 had no effect on the ability of ETAA1-AAD to stimulate ATR kinase activity (Figure S6a-c). Likewise, ETAA1 did not impair the ability of TopBP1-AAD to enhance ATR activity (Figure S6d), suggesting that ETAA1 and TopBP1 function in independent ATR activation pathways. Remarkably, however, whereas individual depletion of TopBP1 or ETAA1 from HCT116 cells only had a modest impact on cellular fitness, simultaneous loss of these proteins profoundly impaired cell proliferation (Figure 6a) and led to strongly increased rates of spontaneous DNA breakage in otherwise undamaged cells, as evidenced by neutral comet assays and markers of ATM kinase activity, including H2AX, RPA2 S4/S8 and Chk2 T68 phosphorylation (Figure 6b-e). This phenotype mimicked the effect of persistent ATR inhibition and could be seen in both HCT116 and HeLa ETAA1 $\triangle$ cells, as well as after siRNA-mediated co-depletion of ETAA1 and TopBP1 (Figure 6b-e;S6e-g). Quantitative image analysis revealed that depletion of TopBP1 from ETAA1 $\triangle$ cells led to strongly elevated levels of chromatin-bound RPA accompanied by $\gamma \mathrm{H} 2 \mathrm{AX}$ formation (Figure $6 \mathrm{c}$ ), suggesting that simultaneous loss of ETAA1 and TopBP1 leads to massive replication fork collapse ${ }^{42}$. A substantial proportion of these cells displayed fragmented nuclei with sub-G1 DNA content 
(Figure 6c,d;S6h), a phenotype also observed after loss of ATR kinase activity ${ }^{15,45}$. Cells lacking both ETAA1 and TopBP1 progressed abnormally through mitosis and frequently stained positive for $\gamma \mathrm{H} 2 \mathrm{AX}$ and the mitotic marker $\mathrm{H} 3 \mathrm{pS} 10$ (Figure S6i;Video S1,S2), suggesting that they have a defective G2/M checkpoint and enter mitosis prematurely in the presence of DNA damage. In contrast, apoptosis inhibitors did not suppress nuclear fragmentation following combined ETAA1 and TopBP1 loss (Figure S6j), suggesting this phenotype mostly results from mitotic catastrophe. Complementation of ETAA1 1 cells with ectopic WT ETAA1, but not AAD- and RPA binding-deficient mutants, reversed Chk2 and RPA2 hyper-phosphorylation resulting from TopBP1 knockdown in this background (Figure 6g). These data suggested that ETAA1 and TopBP1 operate in parallel pathways of ATR regulation, and that their simultaneous inactivation is detrimental to genome integrity and cell survival by causing robust impairment of ATR signaling. Consistently, whereas depletion of ETAA1 or TopBP1 alone did not markedly impact ATR-dependent Chk1 phosphorylation in HCT116 and HeLa cells, simultaneous loss of these proteins strongly reduced Chk1 phosphorylation levels (Figure 6b,g;S6e). Stable expression of ETAA1 WT but not AAD- and RPA binding-deficient mutants restored Chk1 phosphorylation in ETAA1A cells depleted of TopBP1 (Figure 6g). Finally, ETAA1A cells were more sensitive to low concentrations of ATR inhibitors than WT cells (Figure 6h), suggesting that loss of ETAA1 decreases the overall cellular capacity to activate ATR. Collectively, these findings suggest that ETAA1 functions in parallel with, but independent of, the canonical TopBP1 pathway in stimulating ATR kinase activity, and that both pathways make important contributions to ATR-mediated responses to replication stress. 


\section{ETAA1 and TopBP1 jointly promote ATR signaling in cancer cell lines}

To further test the emerging model of ATR activation involving parallel TopBP1- and ETAA1-dependent pathways, we analyzed ETAA1 expression status in a panel of human cancer cell lines. While the abundance of ETAA1 showed considerable variation across cell lines, we noted that U2OS osteosarcoma cells express abnormally low levels of ETAA1, which did not reflect reduced abundance of ETAA1 mRNA compared to other cell lines (Figure 7a;S7a,b). Using the protein synthesis inhibitor Cycloheximide (CHX), we found that unlike in HeLa cells, the halflife of ETAA1 was very short in U2OS cells (Figure S7c), possibly accounting for their low ETAA1 expression levels. This provided a framework to further test the biological relevance of ETAA1 for ATR activation. Strikingly, we found that knockdown of TopBP1 quantitatively suppressed HU-induced Chk1 phosphorylation in U2OS cells but not in HeLa cells that express higher levels of ETAA1 (Figure 7b). In contrast, depleting TopBP1 from U2OS cells stably expressing GFP-ETAA1 had little effect on Chk1 phosphorylation after HU treatment (Figure 7b). Similarly, loss of TopBP1 in U2OS but not HeLa cells markedly impaired ATR-dependent H2AX phosphorylation after HU-induced fork stalling, almost mimicking complete ATR inhibition, and this defect could be at least partially reversed by stable expression of GFP-ETAA1 in U2OS cells (Figure 7b-d). Consistently, combined knockdown of TopBP1 and ETAA1 was necessary to suppress ATR-dependent H2AX phosphorylation after HU treatment in HeLa cells, while depletion of either factor alone had little effect (Figure S7d-f). These findings provide further evidence that ETAA1 and TopBP1 function in independent but converging branches of ATR activation after replication stress (Figure 7e). 


\section{Discussion}

Detailed insights into the molecular framework governing ATR signaling are central to understanding how cells counteract genetic alterations and severe pathologies that may arise as a consequence. Stimulation of ATR kinase activity depends on AADcontaining proteins, three of which contribute to promoting Mec1/ATR-dependent signaling during $\mathrm{S}$ phase in budding yeast ${ }^{30}$. Activation of vertebrate ATR is thought to rely largely on a pathway mediated by TopBP1, the only factor in higher eukaryotes known to harbor an AAD. Here, we have shown that ATR activation is not exclusively mediated by this pathway, but that human cells possess a second ATRstimulatory mechanism that centers on ETAA1 (Figure 7e), a previously uncharacterized protein that undergoes enrichment at DNA damage sites via dual RPA1- and RPA2-binding motifs and contains a bona fide AAD. Similar findings were reported by Cortez and colleagues (Nature Cell Biology, this issue). The ETAA1 AAD is a potent inducer of ATR kinase activation and is essential for the ability of ETAA1 to promote ATR signaling, replication integrity, and cell survival. While the known AADs share little sequence homology, Mec1/ATR activation by these domains involves pairs of aromatic amino acids whose spacing varies substantially among individual $\mathrm{AADs}^{30,46}$. Interestingly, the sequence spanning a conserved aromatic residue within the TopBP1 AAD that is essential for its ability to promote ATR activation ${ }^{26}$ shows homology to a region within ETAA1-AAD, point mutations of which prevent ATR activation by full-length ETAA1. Thus, the TopBP1 and ETAA1 AADs likely stimulate ATR kinase activity by analogous mechanisms.

The combination of RPA-binding and AAD motifs within ETAA1 may render it a potent ATR activator by obviating the need for ancillary factors targeting it to ATR- 
ATRIP complexes at genotoxic stress sites. Indeed, ETAA1 promotes ATR activation independently of the canonical TopBP1/9-1-1 axis, however it remains to be seen whether ETAA1 collaborates with other factors in this process. Studies in mice have shown that the integrity of the TopBP1 AAD is essential for viability ${ }^{28}$. Our data suggest that the ETAA1 and TopBP1 pathways jointly promote ATR signaling, and that both pathways must be inactivated to robustly suppress ATR kinase activity in cells, illustrated by the synthetic lethality caused by combined ETAA1 and TopBP1 loss. An important question arising from these findings concerns the distribution of labor between TopBP1 and ETAA1 in activating ATR after different genotoxic stimuli. Conceivably, they could act on distinct pools of RPA-ssDNA-bound ATRATRIP complexes at DNA damage sites. ETAA1 might be particularly important for stimulating ATR kinase activity when long stretches of RPA-coated ssDNA are produced, such as during DSB end resection, where most ATR-ATRIP would be out of reach of 9-1-1-bound TopBP1 at ssDNA-dsDNA junctions. A similar role in promoting ATR kinase activity away from ssDNA-dsDNA junctions was recently suggested for the MRN component Nbs1, albeit in a manner that required TopBP $1^{41}$. Recent work has shown that two distinct modes of Mec1/ATR signaling exist in yeast, one of which is highly active during normal replication but uncoupled from the canonical replication stress-induced checkpoint signaling pathway involving Mec1/ $\operatorname{Rad} 53^{47}$. It is tempting to speculate that the existence of two AAD-containing proteins in vertebrates may similarly enable different modes of ATR signaling in accordance with the status and integrity of DNA replication.

In preclinical studies, ATR kinase inhibitors have shown promising potential in cancer therapy, exacerbating oncogene-induced replication stress to sensitize cancer 
cells but not normal cells to DNA damage ${ }^{48}$. The functional status of ETAA1 in cancer cells, which typically experience high loads of replication stress ${ }^{49}$, may be an important determinant of their relative sensitivity to ATR inhibitors, since loss of ETAA1 leads to markedly reduced survival rates after treatment with these agents, particularly at low doses. This may be of clinical relevance, as the levels of ETAA1 expression vary considerably among different cancer cell lines and has important bearings on how ATR-dependent signaling responses are instigated in different genetic backgrounds. Of note, variations in the ETAA1 locus are associated with increased pancreatic cancer risk ${ }^{50,51}$. By promoting ATR-dependent responses to replication stress, ETAA1 may be an important factor in the cellular defence against cancer and other diseases associated with genomic instability. 


\section{Acknowledgements}

We thank David Cortez and Akiko Kumagai for providing reagents. We thank Korbinian Mayr, Igor Paron and Gaby Sowa for mass spectrometry support and Martin Steger and Bianca Splettstösser (all from Max Planck Institute of Biochemistry) for experimental advice. This work was supported by grants from The Novo Nordisk Foundation (grants no. NNF14CC0001 and NNF12OC0002114), European Research Council (ERC, grant agreement no. 616236 (DDRegulation)), The Danish Cancer Society, The Danish Council for Independent Research, Danish National Research Foundation (grant no. DNRF115) and Center for Integrated Protein Science Munich (CIPSM). The authors declare no competing financial interests.

\section{Author contributions}

PH and NM conceived the study. PH, SH, MAXT and TH designed and performed cell-, biochemistry-, and imaging-based experiments and analyzed the data, under the supervision of NM. MR designed and performed mass spectrometry experiments and analyzed the data, under the supervision of MM. LIT provided help and support with quantitative image analysis and time-lapse microscopy. SB-J co-supervised the study. NM wrote the manuscript with inputs from $\mathrm{PH}$ and MR. All authors discussed the results and commented on the manuscript.

\section{Competing financial interests}

The authors declare no competing financial interests. 


\section{References}

1. Hoeijmakers, J.H. Genome maintenance mechanisms for preventing cancer. Nature 411, 366-374 (2001).

2. Lindahl, T. \& Barnes, D.E. Repair of endogenous DNA damage. Cold Spring Harb Symp Quant Biol 65, 127-133 (2000).

3. Jackson, S.P. \& Bartek, J. The DNA-damage response in human biology and disease. Nature 461, 1071-1078 (2009).

4. Ciccia, A. \& Elledge, S.J. The DNA damage response: making it safe to play with knives. Mol Cell 40, 179-204 (2010).

5. Matsuoka, S. et al. ATM and ATR substrate analysis reveals extensive protein networks responsive to DNA damage. Science 316, 1160-1166 (2007).

6. Bensimon, A. et al. ATM-dependent and -independent dynamics of the nuclear phosphoproteome after DNA damage. Sci Signal 3, rs3 (2010).

7. Beli, P. et al. Proteomic investigations reveal a role for RNA processing factor THRAP3 in the DNA damage response. Mol Cell 46, 212-225 (2012).

8. Marechal, A. \& Zou, L. DNA damage sensing by the ATM and ATR kinases. Cold Spring Harbor perspectives in biology 5 (2013).

9. Liu, Q. et al. Chk1 is an essential kinase that is regulated by Atr and required for the G(2)/M DNA damage checkpoint. Genes Dev 14, 14481459 (2000).

10. Matsuoka, S., Huang, M. \& Elledge, S.J. Linkage of ATM to cell cycle regulation by the Chk2 protein kinase. Science 282, 1893-1897 (1998).

11. O'Driscoll, M., Ruiz-Perez, V.L., Woods, C.G., Jeggo, P.A. \& Goodship, J.A. A splicing mutation affecting expression of ataxia-telangiectasia and Rad3related protein (ATR) results in Seckel syndrome. Nat Genet 33, 497-501 (2003).

12. Shiloh, Y. \& Ziv, Y. The ATM protein kinase: regulating the cellular response to genotoxic stress, and more. Nat Rev Mol Cell Biol 14, 197-210 (2013).

13. Zeman, M.K. \& Cimprich, K.A. Causes and consequences of replication stress. Nat Cell Biol 16, 2-9 (2014).

14. Cimprich, K.A. \& Cortez, D. ATR: an essential regulator of genome integrity. Nat Rev Mol Cell Biol 9, 616-627 (2008).

15. Brown, E.J. \& Baltimore, D. ATR disruption leads to chromosomal fragmentation and early embryonic lethality. Genes Dev 14, 397-402 (2000).

16. de Klein, A. et al. Targeted disruption of the cell-cycle checkpoint gene ATR leads to early embryonic lethality in mice. Curr Biol 10, 479-482 (2000).

17. Byun, T.S., Pacek, M., Yee, M.C., Walter, J.C. \& Cimprich, K.A. Functional uncoupling of MCM helicase and DNA polymerase activities activates the ATR-dependent checkpoint. Genes Dev 19, 1040-1052 (2005).

18. Zou, L. \& Elledge, S.J. Sensing DNA damage through ATRIP recognition of RPA-ssDNA complexes. Science 300, 1542-1548 (2003).

19. Cortez, D., Guntuku, S., Qin, J. \& Elledge, S.J. ATR and ATRIP: partners in checkpoint signaling. Science 294, 1713-1716 (2001). 
20. Delacroix, S., Wagner, J.M., Kobayashi, M., Yamamoto, K. \& Karnitz, L.M. The Rad9-Hus1-Rad1 (9-1-1) clamp activates checkpoint signaling via TopBP1. Genes Dev 21, 1472-1477 (2007).

21. Lee, J., Kumagai, A. \& Dunphy, W.G. The Rad9-Hus1-Rad1 checkpoint clamp regulates interaction of TopBP1 with ATR. J Biol Chem 282, 2803628044 (2007).

22. Cotta-Ramusino, C. et al. A DNA damage response screen identifies RHINO, a 9-1-1 and TopBP1 interacting protein required for ATR signaling. Science 332, 1313-1317 (2011).

23. Duursma, A.M., Driscoll, R., Elias, J.E. \& Cimprich, K.A. A role for the MRN complex in ATR activation via TOPBP1 recruitment. Mol Cell 50, 116-122 (2013).

24. Zou, L., Cortez, D. \& Elledge, S.J. Regulation of ATR substrate selection by Rad17-dependent loading of Rad9 complexes onto chromatin. Genes Dev 16, 198-208 (2002).

25. Parrilla-Castellar, E.R., Arlander, S.J. \& Karnitz, L. Dial 9-1-1 for DNA damage: the Rad9-Hus1-Rad1 (9-1-1) clamp complex. DNA Repair (Amst) 3, 1009-1014 (2004).

26. Kumagai, A., Lee, J., Yoo, H.Y. \& Dunphy, W.G. TopBP1 activates the ATRATRIP complex. Cell 124, 943-955 (2006).

27. Mordes, D.A., Glick, G.G., Zhao, R. \& Cortez, D. TopBP1 activates ATR through ATRIP and a PIKK regulatory domain. Genes Dev 22, 1478-1489 (2008).

28. Zhou, Z.W. et al. An essential function for the ATR-activation-domain (AAD) of TopBP1 in mouse development and cellular senescence. PLoS Genet 9, e1003702 (2013).

29. Navadgi-Patil, V.M. \& Burgers, P.M. The unstructured C-terminal tail of the 9-1-1 clamp subunit Ddc1 activates Mec1/ATR via two distinct mechanisms. Mol Cell 36, 743-753 (2009).

30. Kumar, S. \& Burgers, P.M. Lagging strand maturation factor Dna2 is a component of the replication checkpoint initiation machinery. Genes Dev 27, 313-321 (2013).

31. Raschle, M. et al. Proteomics reveals dynamic assembly of repair complexes during bypass of DNA cross-links. Science 348 (2015).

32. Borowski, A. et al. Structure and function of ETAA16: a novel cell surface antigen in Ewing's tumours. Cancer Immunol Immunother 55, 363-374 (2006).

33. Bekker-Jensen, S. et al. Spatial organization of the mammalian genome surveillance machinery in response to DNA strand breaks. J Cell Biol 173, 195-206 (2006).

34. Symington, L.S. \& Gautier, J. Double-strand break end resection and repair pathway choice. Annu Rev Genet 45, 247-271 (2011).

35. Mer, G. et al. Structural basis for the recognition of DNA repair proteins UNG2, XPA, and RAD52 by replication factor RPA. Cell 103, 449-456 (2000).

36. Ciccia, A. et al. The SIOD disorder protein SMARCAL1 is an RPAinteracting protein involved in replication fork restart. Genes Dev 23, 2415-2425 (2009). 
37. Unsal-Kacmaz, K. et al. The human Tim/Tipin complex coordinates an Intra-S checkpoint response to UV that slows replication fork displacement. Mol Cell Biol 27, 3131-3142 (2007).

38. Xu, X. et al. The basic cleft of RPA70N binds multiple checkpoint proteins, including RAD9, to regulate ATR signaling. Mol Cell Biol 28, 7345-7353 (2008).

39. Toledo, L.I., Murga, M., Gutierrez-Martinez, P., Soria, R. \& FernandezCapetillo, 0. ATR signaling can drive cells into senescence in the absence of DNA breaks. Genes Dev 22, 297-302 (2008).

40. Ward, I.M. \& Chen, J. Histone H2AX is phosphorylated in an ATRdependent manner in response to replicational stress. J Biol Chem 276, 47759-47762 (2001).

41. Shiotani, B. et al. Two distinct modes of ATR activation orchestrated by Rad17 and Nbs1. Cell reports 3, 1651-1662 (2013).

42. Toledo, L.I. et al. ATR prohibits replication catastrophe by preventing global exhaustion of RPA. Cell 155, 1088-1103 (2013).

43. Couch, F.B. et al. ATR phosphorylates SMARCAL1 to prevent replication fork collapse. Genes Dev 27, 1610-1623 (2013).

44. Lukas, C. et al. 53BP1 nuclear bodies form around DNA lesions generated by mitotic transmission of chromosomes under replication stress. Nat Cell Biol 13, 243-253 (2011).

45. Toledo, L.I. et al. A cell-based screen identifies ATR inhibitors with synthetic lethal properties for cancer-associated mutations. Nat Struct Mol Biol 18, 721-727 (2011).

46. Wanrooij, P.H., Tannous, E., Kumar, S., Navadgi-Patil, V.M. \& Burgers, P.M. Probing the Mec1ATR Checkpoint Activation Mechanism with Small Peptides. J Biol Chem 291, 393-401 (2016).

47. Bastos de Oliveira, F.M. et al. Phosphoproteomics reveals distinct modes of Mec1/ATR signaling during DNA replication. Mol Cell 57, 1124-1132 (2015).

48. Fokas, E. et al. Targeting ATR in DNA damage response and cancer therapeutics. Cancer Treat Rev 40, 109-117 (2014).

49. Lopez-Contreras, A.J. \& Fernandez-Capetillo, O. The ATR barrier to replication-born DNA damage. DNA Repair (Amst) 9, 1249-1255 (2010).

50. Childs, E.J. et al. Common variation at 2p13.3, 3q29, 7p13 and 17q25.1 associated with susceptibility to pancreatic cancer. Nat Genet 47, 911-916 (2015).

51. Wu, C. et al. Genome-wide association study identifies five loci associated with susceptibility to pancreatic cancer in Chinese populations. Nat Genet 44, 62-66 (2012). 


\section{Figure legends}

\section{Figure 1.}

\section{Systematic profiling of DSB-containing chromatin reveals ETAA1 as a potential}

\section{DDR factor}

a. CHROMASS workflow to analyze protein recruitment to DSB-containing chromatin. Sperm chromatin was incubated in Xenopus egg extract and DSBs induced by addition of PflMI enzyme. After various times, chromatin was isolated by centrifugation through a sucrose cushion and analyzed by label-free mass spectrometry. b. Heatmap showing the mean of the z-scored LFQ protein intensities of selected factors for DSB-containing chromatin normalized to the undamaged control $(n=3)$. See Table S2 for recruitment profiles for all quantified proteins. c. Protein recruitment to DSB-containing chromatin at 15 min compared to an undamaged control. The volcano plot shows the mean difference of the protein intensity $(\log 2)$ plotted against the $\mathrm{p}$-value resulting from a one-sided modified t-test (see Methods, $n=3$ for both groups). Dashed line indicates the significance cut-off. $\mathbf{d}$. Protein recruitment to DSB-containing chromatin at $45 \mathrm{~min}$, plotted as in (c). e. U2OS cells stably expressing GFP-ETAA1 were subjected to laser microirradiation, fixed 1 h later and co-immunostained with RPA1 and $\gamma \mathrm{H} 2 \mathrm{AX}$ antibodies. f. Quantification of data in (e) (mean $\pm \mathrm{SD} ; n=3 ; 80$ cells analyzed per experiment). g. U2OS cells stably expressing GFP-ETAA1 were treated with CPT $(1 \mathrm{~h})$ or HU $(3 \mathrm{~h})$, fixed and coimmunostained with RPA1 and $\gamma \mathrm{H} 2 \mathrm{AX}$ antibodies. All scale bars, $10 \mu \mathrm{m}$. Data are representative of three (e) and two (g) independent experiments with similar results.

\section{Figure 2.}




\section{ETAA1 is recruited to DNA damage sites through interaction with RPA}

a. Representative images of U2OS cells stably expressing GFP-ETAA1 that were transfected with indicated siRNAs and subjected to laser microirradiation, followed by co-immunostaining with RPA1 and $\gamma \mathrm{H} 2 \mathrm{AX}$ antibodies. Knockdown efficiency of siRNAs is shown in Figure S2a. b. U2OS cells transfected with constructs encoding indicated versions of GFP-ETAA1 or empty vector (EV) were subjected to GFP immunoprecipitation (IP) followed by immunoblotting for the indicated proteins. $\mathbf{c}$. As in (b), except that cells were transfected with indicated GFP-RPA2 constructs or empty vector (EV). SMARCAL1 is a positive control for RPA2 binding. d. Schematic diagram of human ETAA1 protein, indicating the locations of conserved RPA1- and RPA2-binding motifs (RBM1 and RBM2). Alignments of these sequences with RPA1/2-binding motifs from other proteins are shown. Conserved key residues within the RPA-binding motifs are highlighted. e. Representative images of U2OS cells stably expressing wild-type (WT) and mutant forms of GFP-ETAA1 subjected to laser microirradiation followed by immunostaining with $\gamma \mathrm{H} 2 \mathrm{AX}$ antibody. $\mathbf{f}$.

Quantification of data in (e) (mean $\pm \mathrm{SD} ; n=3$; approx. 200 cells counted per condition). All scale bars, $10 \mu \mathrm{m}$. Data are representative of three $(\mathbf{a}, \mathbf{b}, \mathbf{e})$ and two $(\mathbf{c})$ independent experiments with similar results. Uncropped blots $(\mathbf{b}, \mathbf{c})$ are shown in Figure S8.

\section{Figure 3.}

\section{ETAA1 contains an ATR-activating domain (AAD)}

a. U2OS cells transfected with indicated GFP-ETAA1 constructs or empty vector (EV) expressing GFP only were immunostained with $\gamma \mathrm{H} 2 \mathrm{AX}$ antibody. b.

Quantification of data in (a) (mean $\pm \mathrm{SD} ; n=3 ; 240$ cells counted per condition). $\mathbf{c}$. Schematic diagram of human ETAA1 protein, indicating location of the ATR- 
activating domain (AAD), its sequence similarity to the TopBP1 AAD, and the construct used for inducible nuclear translocation of ETAA1-AAD upon 4-OHT treatment. d. U2OS cells expressing GFP-ETAA1-AAD-ERT2 were treated or not with 4-OHT for $2 \mathrm{~h}$ and immunostained with $\gamma \mathrm{H} 2 \mathrm{AX}$ antibody. e. Cells in (d) were analyzed by quantitative imaging (bottom). Representative images are shown (top). $\mathbf{f}$. As in (e), except that cells were treated with indicated kinase inhibitors $10 \mathrm{~min}$ before addition of $4-\mathrm{OHT}$ for $1 \mathrm{~h}$ (mean $\pm \mathrm{SD} ; n=3 ;>3000$ cells analyzed per condition). $\mathbf{g}$. U2OS cells transfected with empty vector (EV) or GFP-ETAA1 expression construct were subjected to GFP immunoprecipitation (IP) followed by immunoblotting for the indicated proteins. h. In vitro kinase assay, using purified ATR-ATRIP complex, recombinant ETAA1-AAD and MCM2(79-138) fragments (the latter serving as ATR model substrate) and radioactive ATP $\left({ }^{32} \mathrm{P}\right)$. Reactions were resolved on SDS-PAGE, stained with Colloidal Blue (CB), and ${ }^{32} \mathrm{P}$ incorporation was assessed by autoradiography. i. Phospho-proteomic analysis of SILAC-labeled U2OS/GFPETAA1-AAD-ERT2 cells treated or not with 4-OHT for $2 \mathrm{~h}$ to monitor phosphorylation changes triggered by forced nuclear expression of ETAA1-AAD. To identify ATR-dependent changes, ATR inhibitor (ATRi) or vehicle was added 15 min before 4-OHT treatment in an independent SILAC experiment (see Figure S3h for experimental set-up). Scatterplot shows the $\log 2$ of mean SILAC ratios from four independent replicates of each experiment plotted against each other (see Table S4 for full data). j. Immunoblot analysis of U2OS/GFP-ETAA1-AAD-ERT2 cells treated with 4-OHT for indicated times in the absence or presence of ATR inhibitor (ATRi). All scale bars, $10 \mu \mathrm{m}$. Data are representative of three $(\mathbf{a}, \mathbf{d}, \mathbf{e}, \mathbf{h})$ and two $(\mathbf{g , j})$ independent experiments with similar results. Uncropped blots (g-j) are shown in Figure S8. 


\section{Figure 4.}

\section{ETAA1 promotes cell survival and ATR signaling after replication stress}

a. Immunoblot analysis of parental HCT116 (WT) cells and derivative ETAA1 knockout lines (ETAA1A) exposed to CPT for various times. Asterisk demarcates an unspecific band detected by the ETAA1 antibody. b. Chromatin-enriched and soluble fractions of HCT116 WT and ETAA1A cell lines left untreated or exposed to CPT for 90 min were immunoblotted with indicated antibodies. c. HCT116 WT and ETAA1 cell lines left untreated or exposed to CPT were co-immunostained with RPA2 and RPA2-pS4/S8 antibodies. d. As in (c), except that cells were co-immunostained with $\gamma \mathrm{H} 2 \mathrm{AX}$ and RPA1 antibodies. e. Quantification of data in (d). $\gamma \mathrm{H} 2 \mathrm{AX}$-negative and positive cell populations were gated and analyzed for mean RPA1 chromatin loading per nucleus by quantitative imaging. Box plot shows median, upper and lower quartiles, 95\% values (whiskers) and outliers ( $n=2000$ cells per condition). f. Clonogenic survival of HCT116 WT, HCT116 ETAA1A, and HCT116 ETAA1A cells stably reconstituted with GFP-ETAA1 that were subjected to various doses of HU for $24 \mathrm{~h}($ mean $\pm \mathrm{SEM} ; n=3)$. g. As in (f), except that cells were subjected to indicated doses of MMC (mean $\pm \mathrm{SEM} ; n=3)$. h. As in (f), except that cells were treated with indicated doses of CPT (mean \pm SEM; $n=3)$. All scale bars, $10 \mu \mathrm{m}$. Data are representative of three $(\mathbf{a}, \mathbf{b})$ and two (c-e) independent experiments with similar results. Uncropped blots $(\mathbf{a}, \mathbf{b})$ are shown in Figure S8.

\section{Figure 5.}

ETAA1 promotes DNA replication integrity during unperturbed and stressful conditions 
a. DNA fiber analysis of HCT116 WT and ETAA1 $\triangle$ cells labeled with CldU (20 min; red) followed by IdU (20 min; green). Fork speeds were calculated as length of labeled track divided by pulse time. Red bars denote median fork speed (400 fibers, pooled from two independent experiments, analyzed per condition). Representative fibers are shown. b. Fork symmetry was calculated as the percentage of shorter divided by longer tracks from (a). Concordance is $100 \%$, representing fully bidirectional replication and equal rates of elongation for both daughter forks. Red bars denote median fork symmetry rate (50 bidirectional forks, pooled from two independent experiments, analyzed per condition). Representative fibers displaying fork symmetrical and asymmetrical bidirectional replication forks are shown. c. As in (a), except that the proportion of new origins (IdU-only tracks) was determined (200 fibers analyzed per condition per experiment). d. Quantitative image analysis of HCT116 cells treated with indicated siRNAs and immunostained with $\gamma \mathrm{H} 2 \mathrm{AX}$ antibody. The proportion of $\gamma \mathrm{H} 2 \mathrm{AX}$-positive cells (pink, arbitrary cut-off) is indicated ( $n=2000$ cells per condition). e. DNA fiber analysis of HCT116 WT and ETAA1A cells labeled with CldU (20 min) followed by IdU and CPT (40 min). New origin firing was determined as in (c) (200 fibers analyzed per condition per experiment). f. Fork progression (CldU- and IdU-positive tracks) in cells from (e). g. HCT116 WT and ETAA1A cells left untreated or grown in the presence of Aphidicolin $(0.2 \mu \mathrm{M})$ for $24 \mathrm{~h}$ were stained with DAPI, and nuclear aberrations (right, representative examples) were assessed (mean $\pm \mathrm{SD} ; n=3$; at least 200 cells were counted per condition). $\mathbf{h}$. As in (g), except that cells were co-immunostained with 53BP1 and Cyclin A antibodies. 53BP1 bodies in Cyclin A-negative (G1 phase) cells (representative examples shown, right) were enumerated (red lines, median; 150 cells analyzed per condition). Data are 
representative of three $(\mathbf{d}, \mathbf{g}, \mathbf{h})$ independent experiments with similar results. Source data (a-c,e,f,h) are provided in Table S5.

\section{Figure 6.}

\section{ETAA1 and TopBP1 promote parallel, but independent, pathways of ATR activation}

a. Colony formation assay, using HCT116 WT and ETAA1 $\triangle$ cell lines transfected with non-targeting control (CTRL) or TopBP1 siRNAs (mean $\pm \mathrm{SD} ; n=3)$. b. Immunoblot analysis of HCT116 WT and ETAA1A cells transfected with TopBP1 siRNAs for the indicated times. Asterisk demarcates an unspecific band detected by the ETAA1 antibody. c. HCT116 WT and ETAA1A cell lines transfected with nontargeting control (CTRL) or TopBP1 siRNAs were analyzed by quantitive imaging. The color of each dot (cell nucleus) represents the mean $\gamma \mathrm{H} 2 \mathrm{AX}$ intensity (low: grey; high: red); $>2000$ cells analyzed per condition. $\mathbf{d}$. Representative images from the experiment shown in (c). Scale bar, $10 \mu \mathrm{m}$. e. DSB formation in cells in (b) was monitored by the neutral Comet assay. Tail lengths of at least 70 nuclei were measured for each condition. f. DNA content of cells in (d) was analyzed by quantitative imaging. The proportion of cells with sub-G1 DNA content (pink) is indicated. g. HCT116 WT, HCT116 ETAA1A-1 and HCT116 ETAA1A-1 cells stably reconstituted with indicated GFP-ETAA1 expression constructs were transfected with non-targeting control $(-)$ or TopBP1 siRNA and immunoblotted with indicated antibodies. h. Viability of HCT116 WT and ETAA1 $\triangle$ cell lines grown in the presence of various doses of ATR inhibitor (ATRi) was assayed by measuring cell growth with the SRB assay $($ mean $\pm \mathrm{SD} ; n=3) .{ }^{*} \mathrm{p}<0.05$, multiple t-test corrected with Holm-Sidak 
method. Data are representative of three $(\mathbf{b}, \mathbf{d}, \mathbf{f}, \mathbf{g})$ and two $(\mathbf{c}, \mathbf{e})$ independent experiments with similar results. Source data (e) are provided in Table S5. Uncropped blots $(\mathbf{b}, \mathbf{g})$ are shown in Figure S8.

\section{Figure 7.}

\section{ETAA1 and TopBP1 both contribute to ATR signaling in cancer cell lines}

a. Immunoblot analysis of ETAA1 and other proteins involved in ATR activation in indicated exponentially growing human cancer cell lines and Tig3 diploid embryonic lung fibroblasts. b. HeLa, U2OS and U2OS/GFP-ETAA1 cells were transfected with TopBP1 siRNAs, treated with HU for $3 \mathrm{~h}$ and processed for immunoblotting with indicated antibodies. c. HeLa and U2OS cells transfected with indicated siRNAs were treated with HU and ATR inhibitor (ATRi) for $3 \mathrm{~h}$ as indicated. Cells were then coimmunostained with $\gamma \mathrm{H} 2 \mathrm{AX}$ and RPA1 antibodies and analyzed by quantitative imaging ( $n=1500$ cells per condition). The contribution of ATR to the $\gamma \mathrm{H} 2 \mathrm{AX}$ signal, based on the ATRi-treated sample, is indicated in blue. Cells displaying maximal HUinduced RPA chromatin loading accompanied by ATM-dependent but ATRindependent $\mathrm{H} 2 \mathrm{AX}$ hyperphosphorylation reflecting fork breakage ${ }^{42}$ are indicated in pink. d. As in (c), but using U2OS and U2OS/GFP-ETAA1 cells ( $n=1500$ cells per condition). e. Model of parallel ETAA1- and TopBP1-mediated stimulation of ATR kinase activity at RPA-coated ssDNA regions (see main text for details). Change of color represents ATR activation. Data are representative of three (b) and two (a,c,d) independent experiments with similar results. Uncropped blots $(\mathbf{a}, \mathbf{b})$ are shown in Figure S8. 


\section{Haahr et al. Figure 1}

a
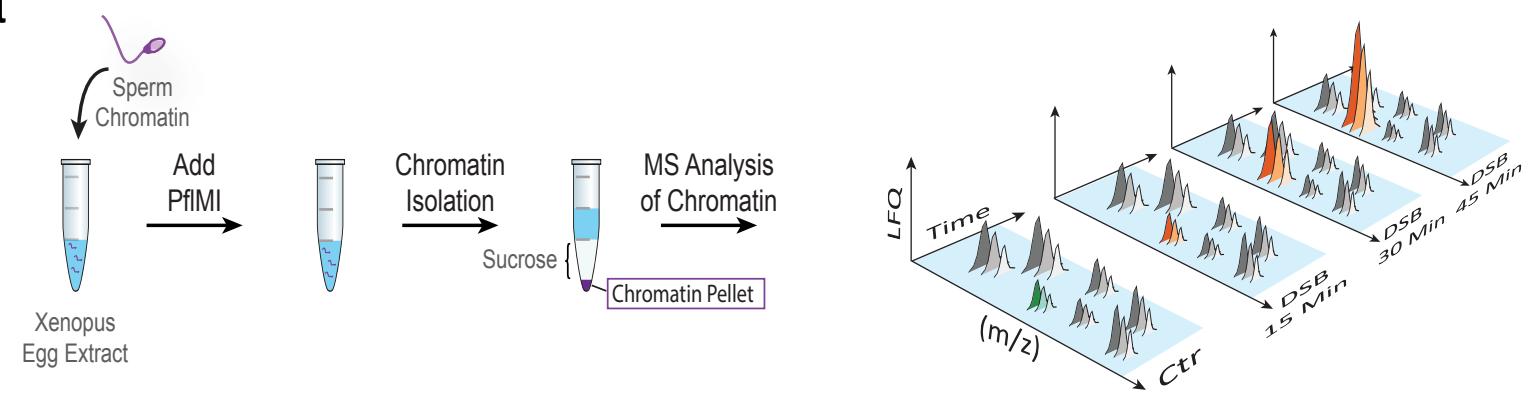

b

Time after RE addition

51015304560 Min

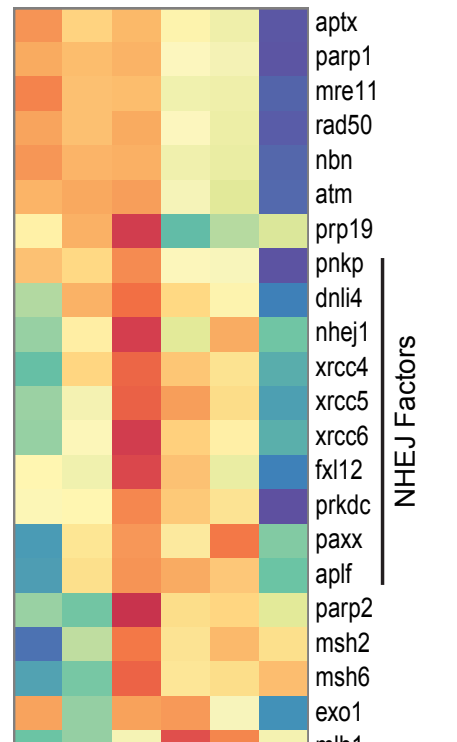

C Recruitment to DSBs (15 min)

d
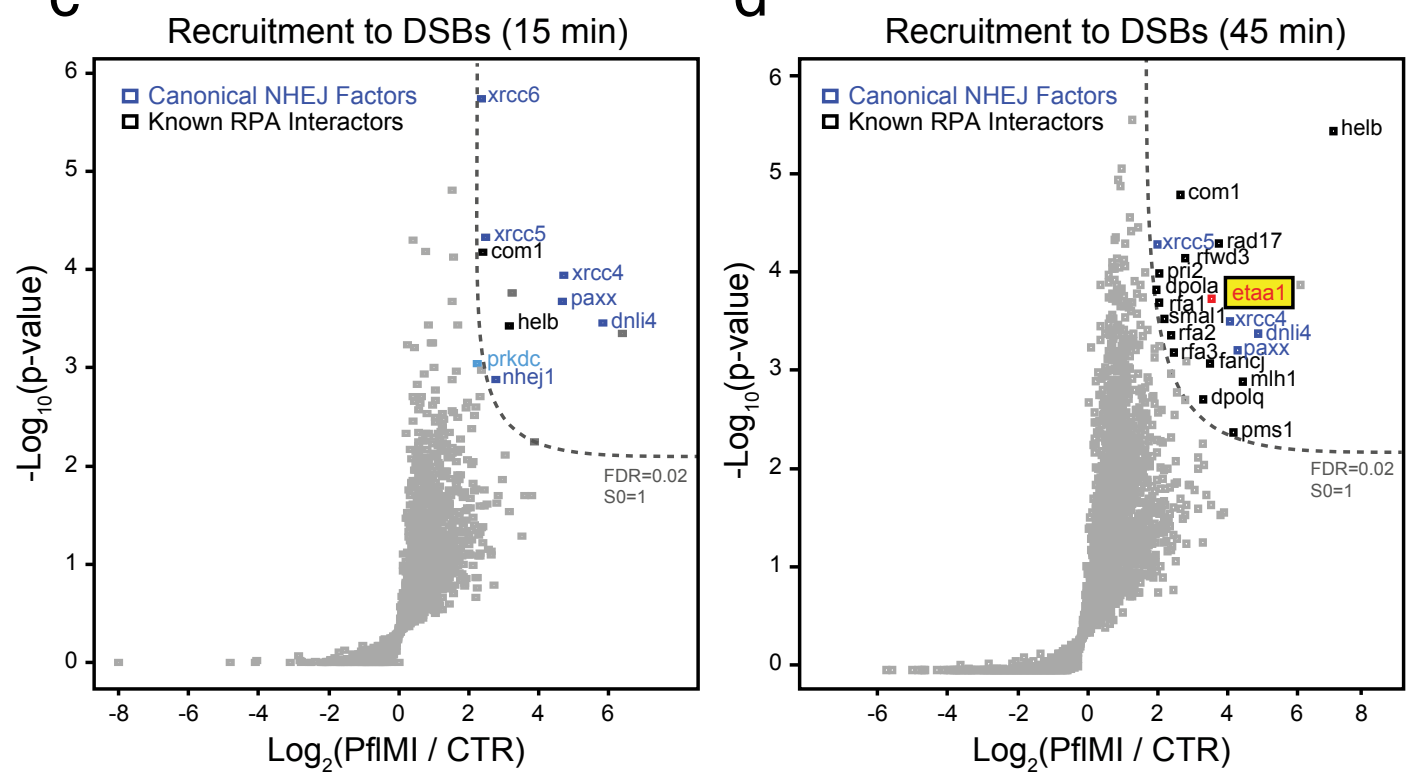

e GFP-ETAA1

RPA1
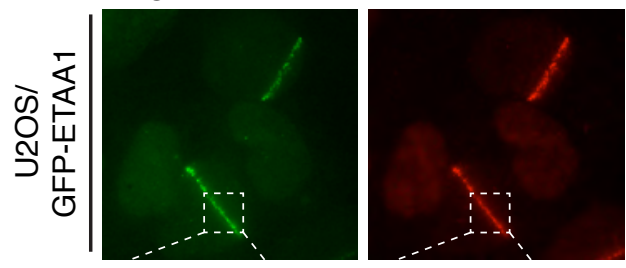

$y H 2 A X$
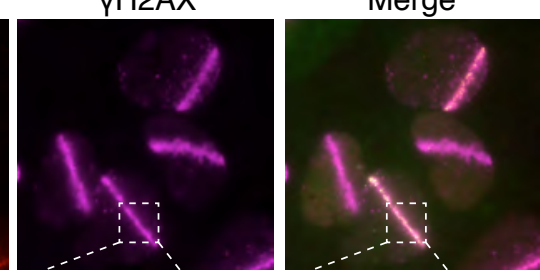

DAPI
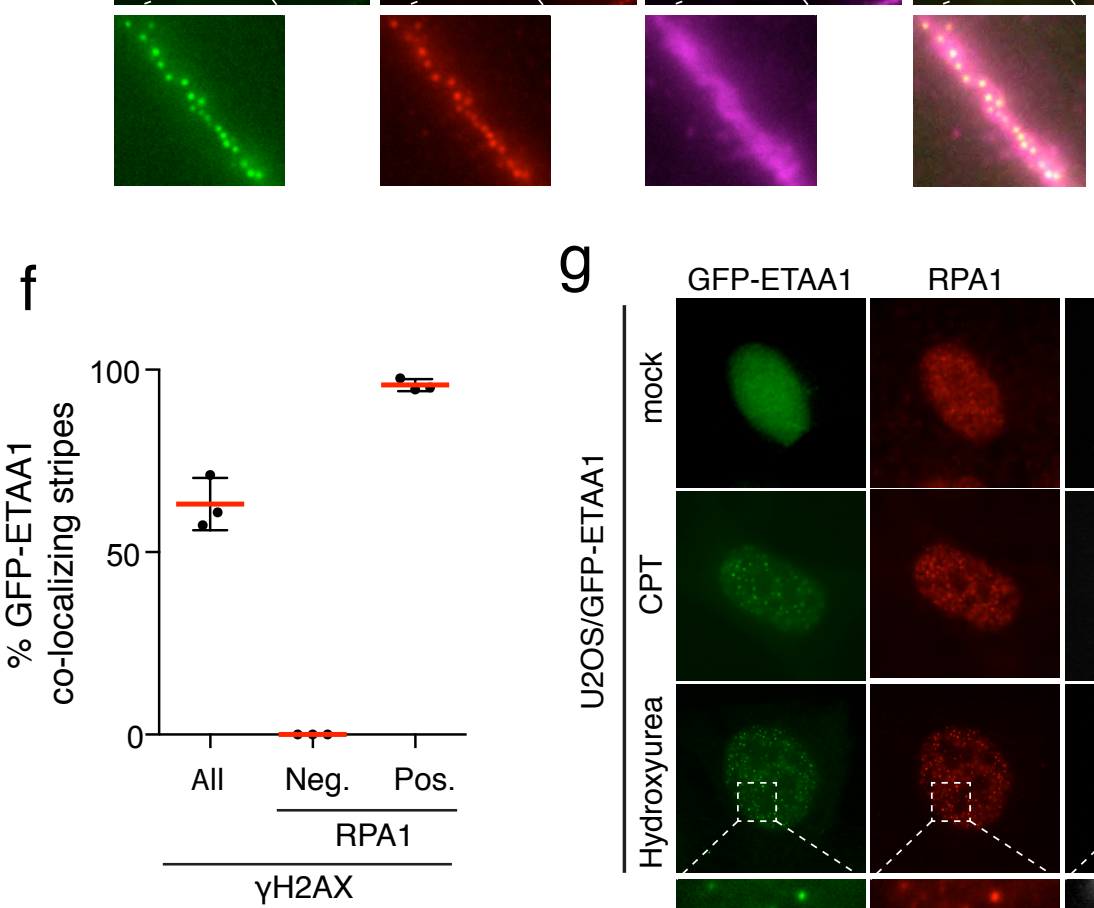

g

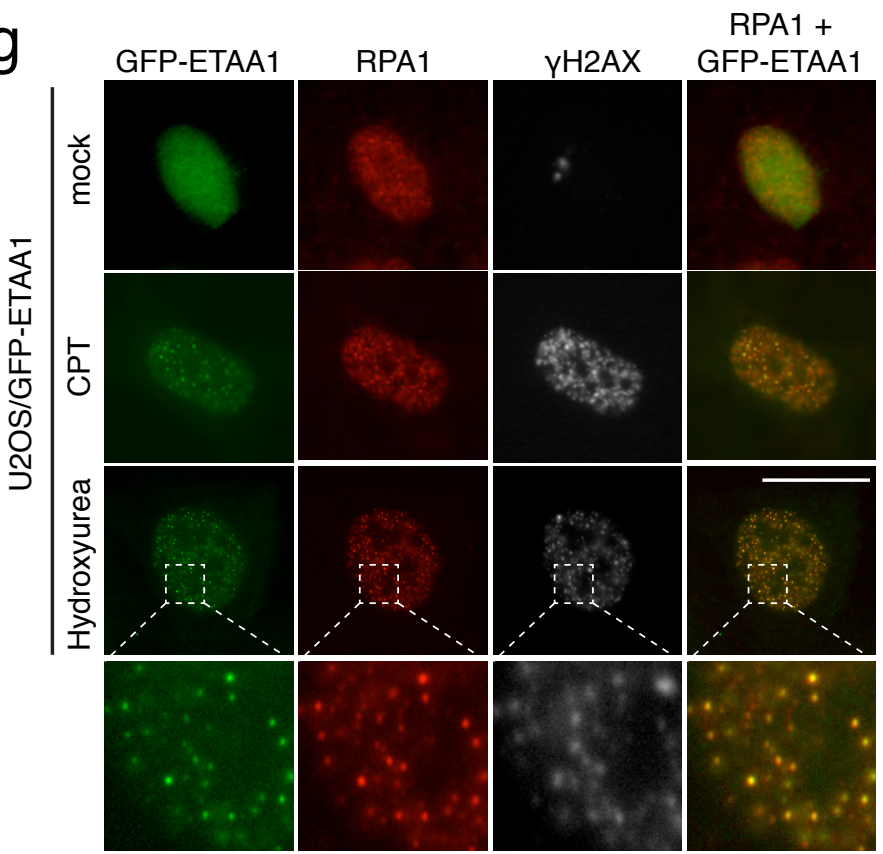




\section{Haahr et al. Figure 2}
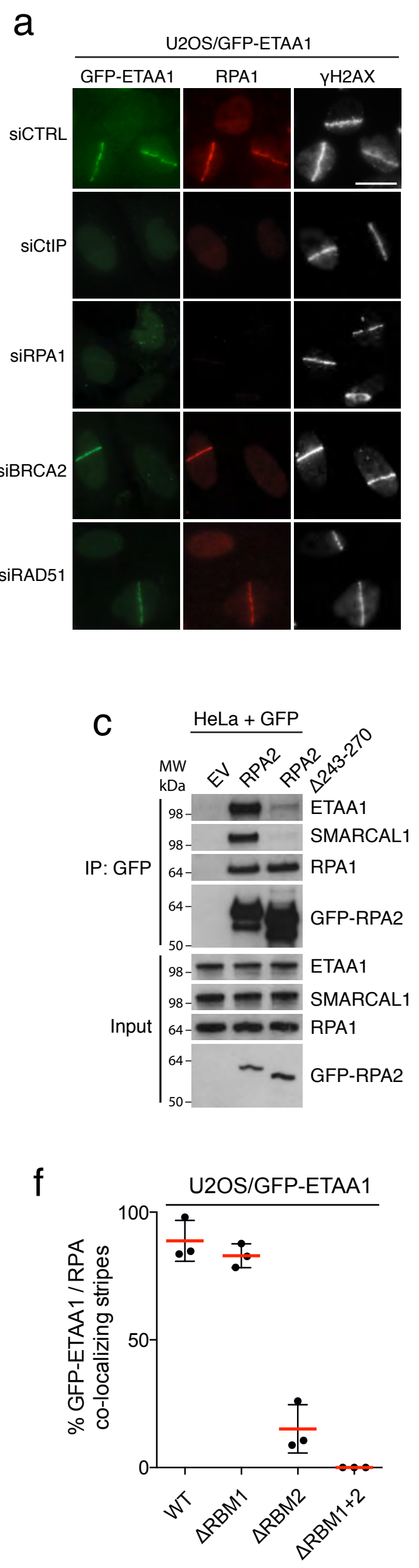
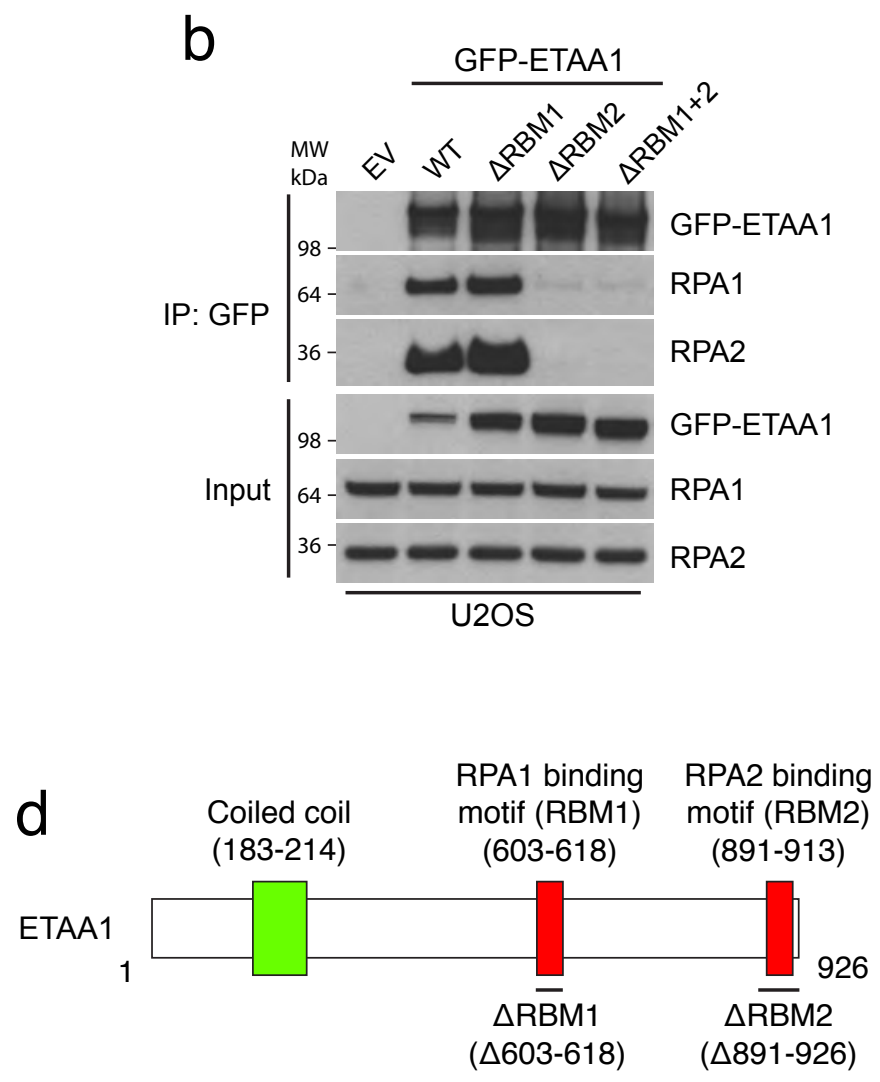

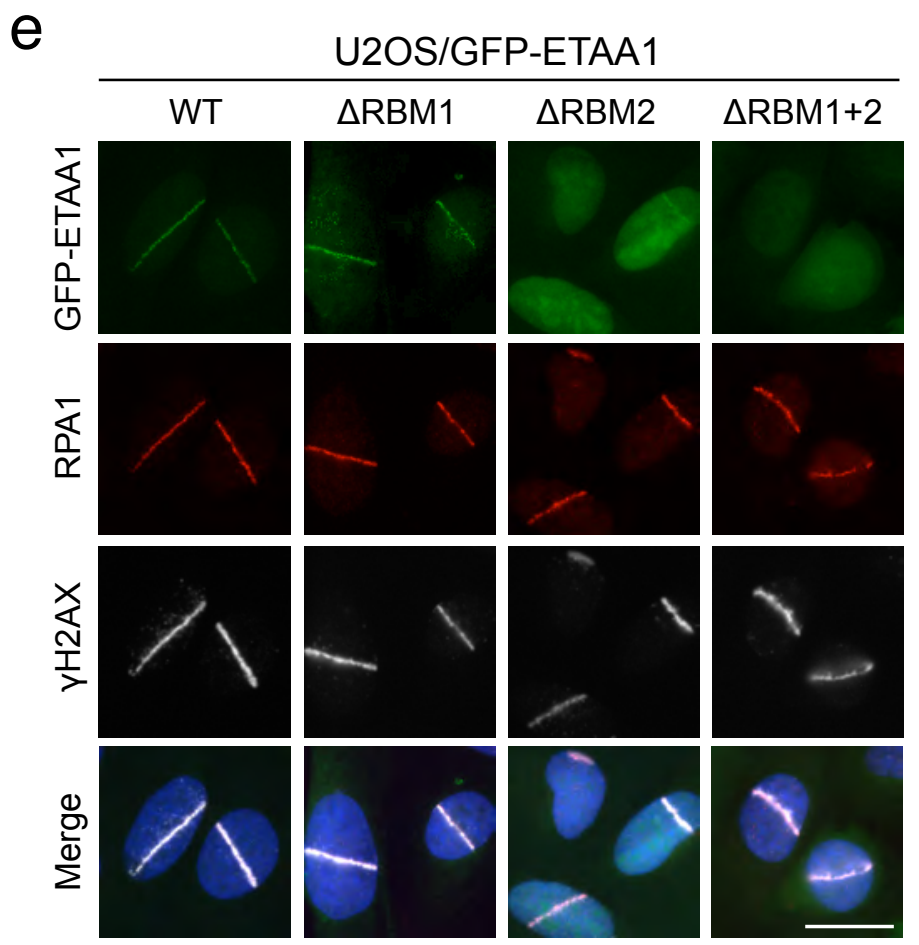


a

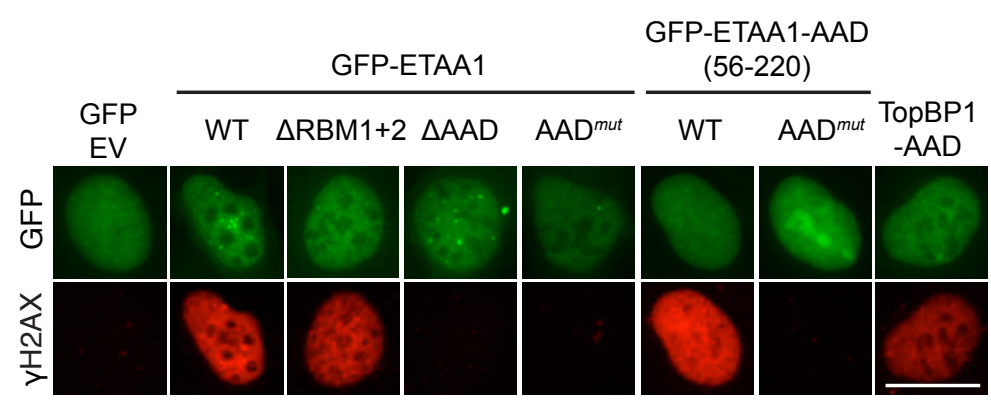

C ATR Activating

Domain (AAD)

(56-220)

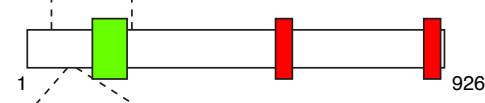

‘ $\quad$ 'FW(106-7)AA = AAD ${ }^{\text {mut }}$

DPDGQNDIFWDQNSPLTKQ̀ hETAA1(98-)

EPSQNEQIIWDDPTAREER hTOPBP 1(1136-)

$: * .::: *: * *: \quad: \quad:$ :

\section{GFP-ETAA1-AAD-ERT2}

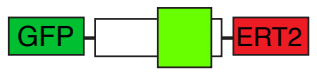

e

U2OS/GFP-ETAA1-AAD-ERT2
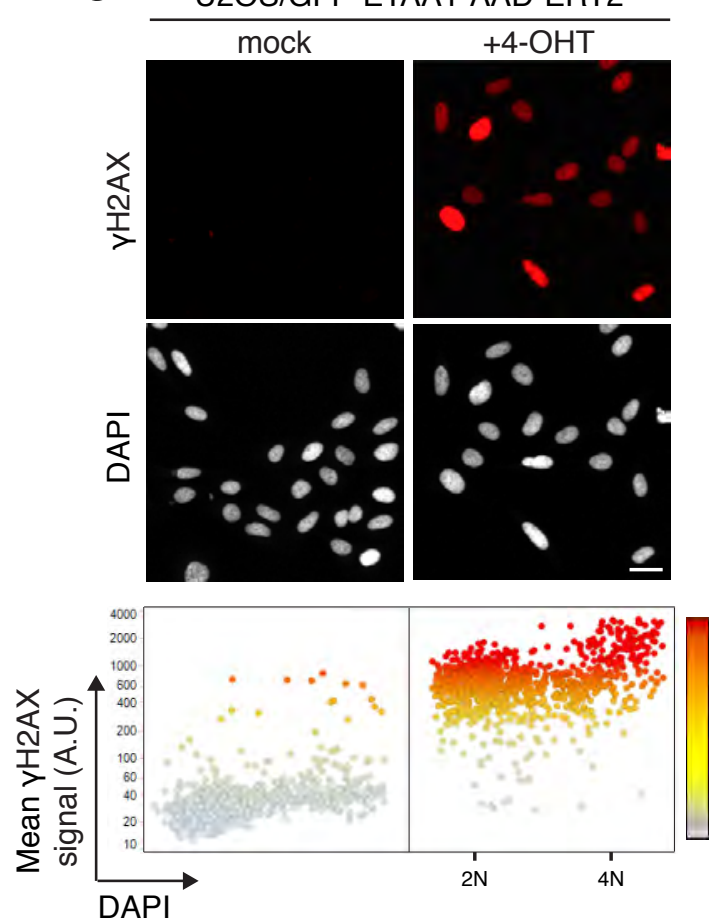

U2OS/GFP-

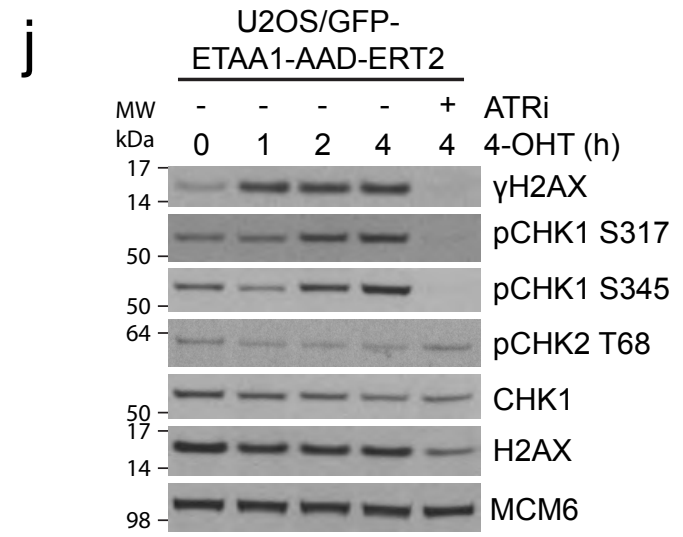

Haahr et al. Figure 3

b
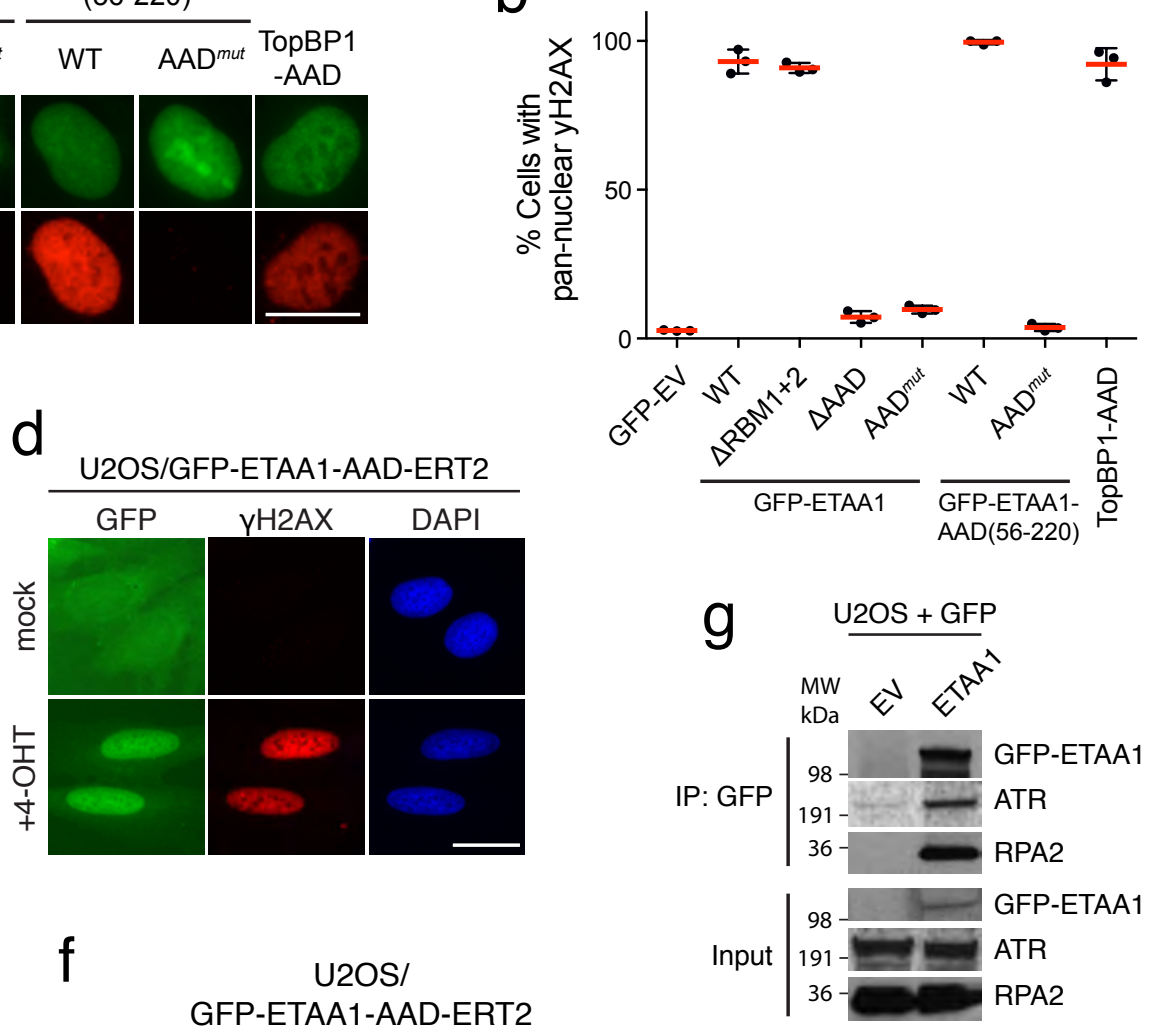

h
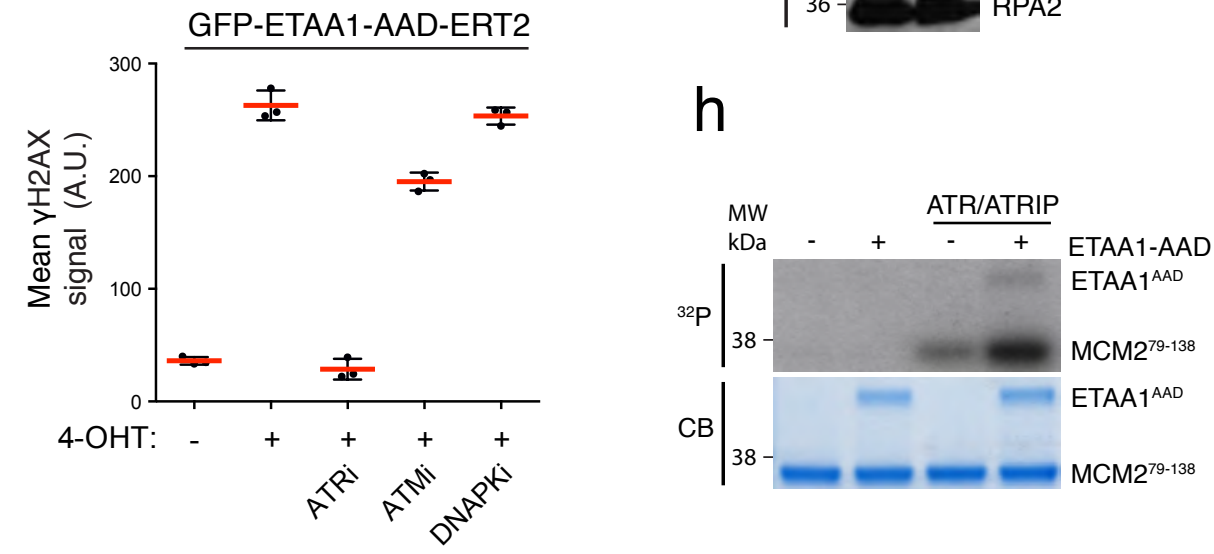

i Identified Phosphosites

(induced by nuclear expression of GFP-ETAA1-AAD-ERT2)

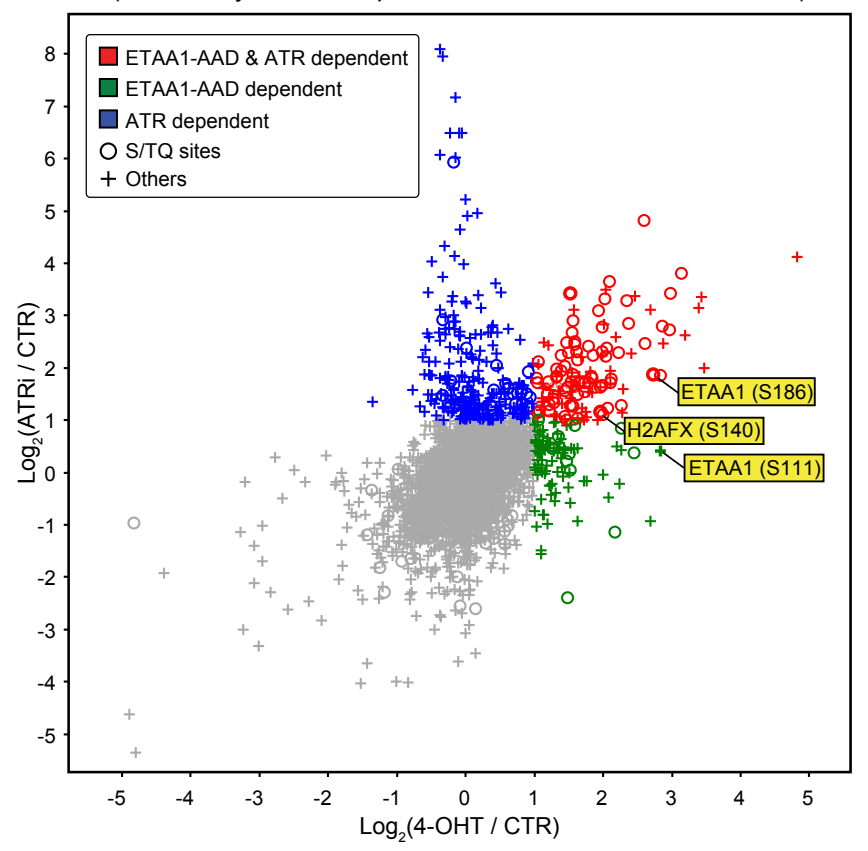




\section{Haahr et al. Figure 4}

a

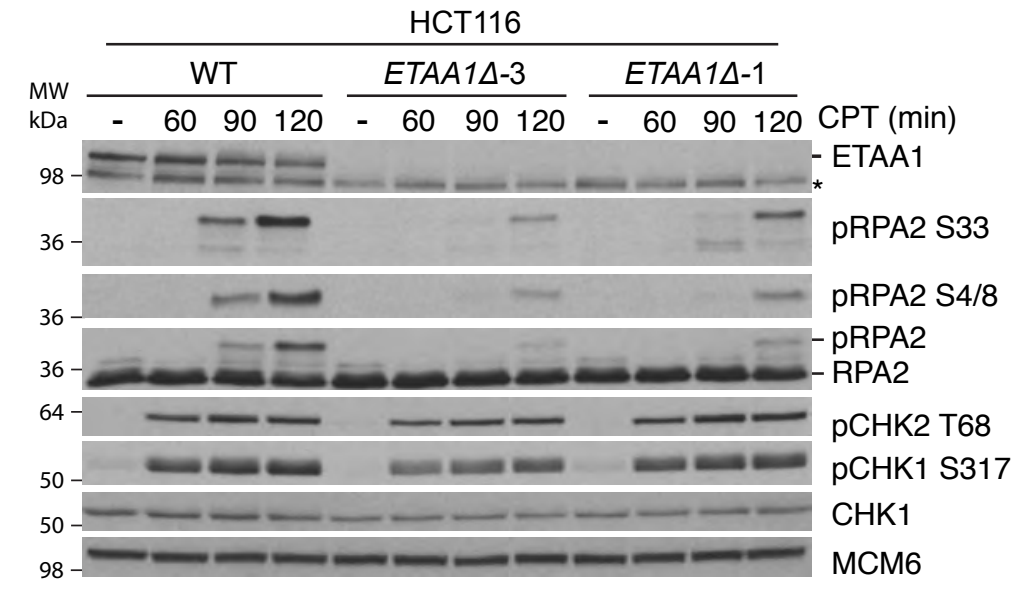

C

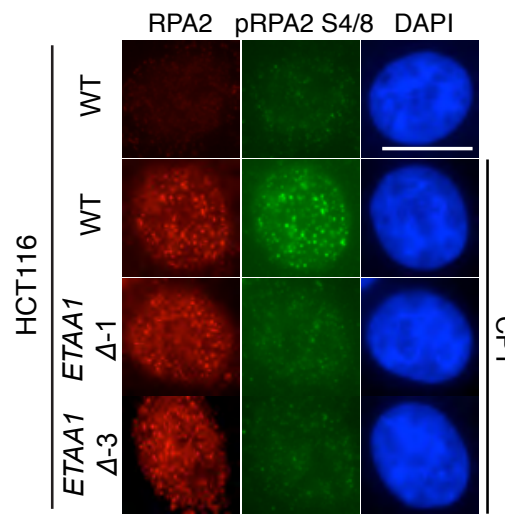

d

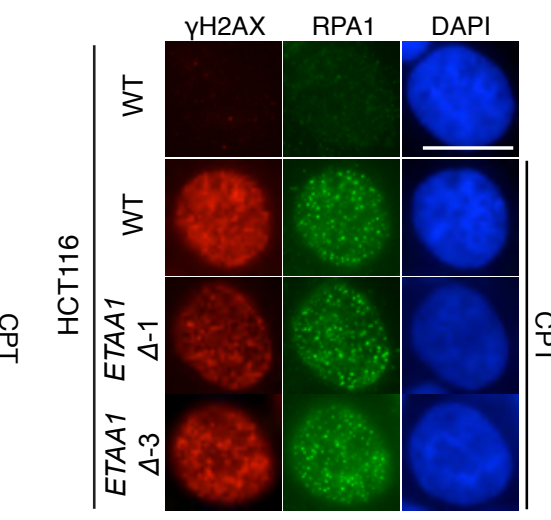

b
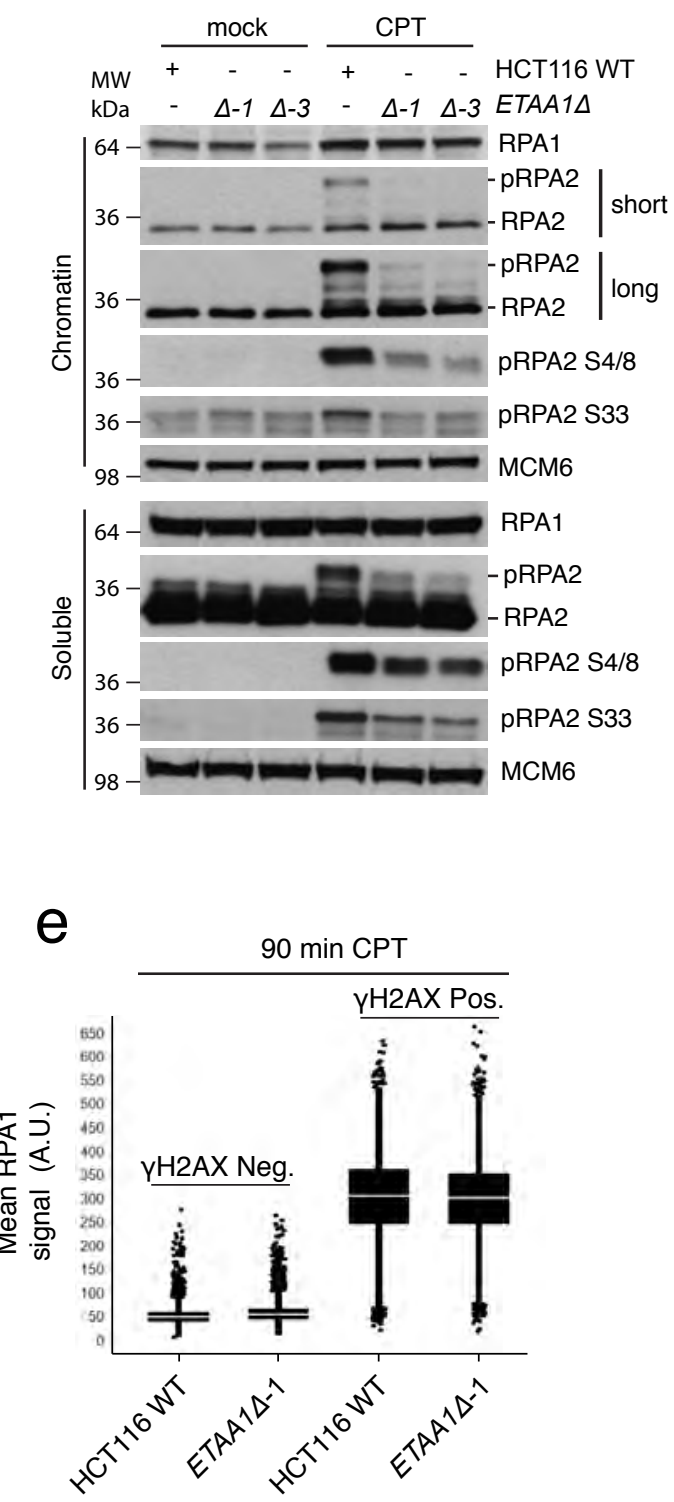

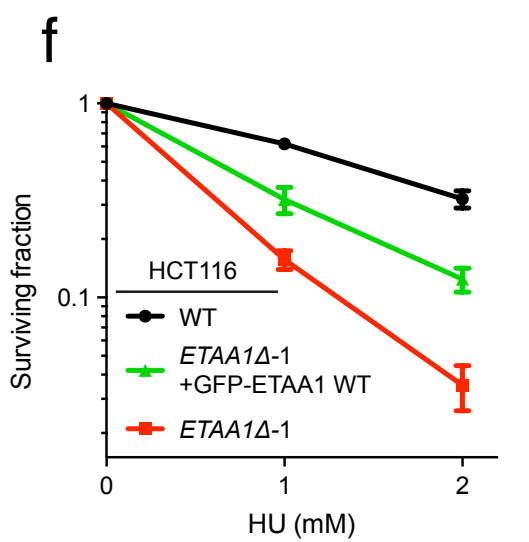

g

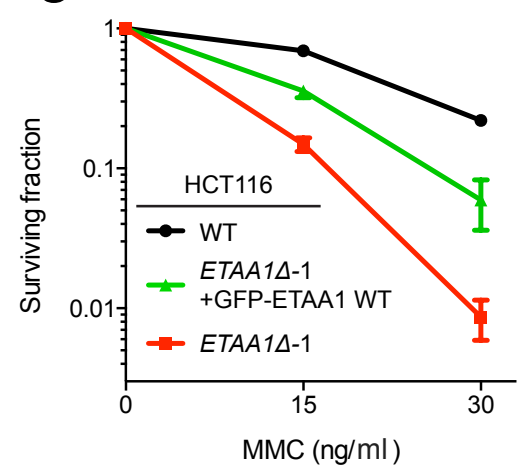

h

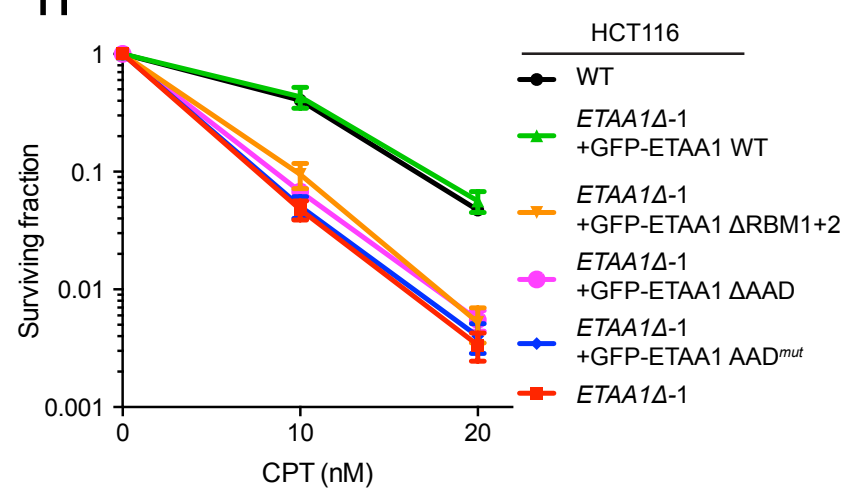




\section{Haahr et al. Figure 5}

a

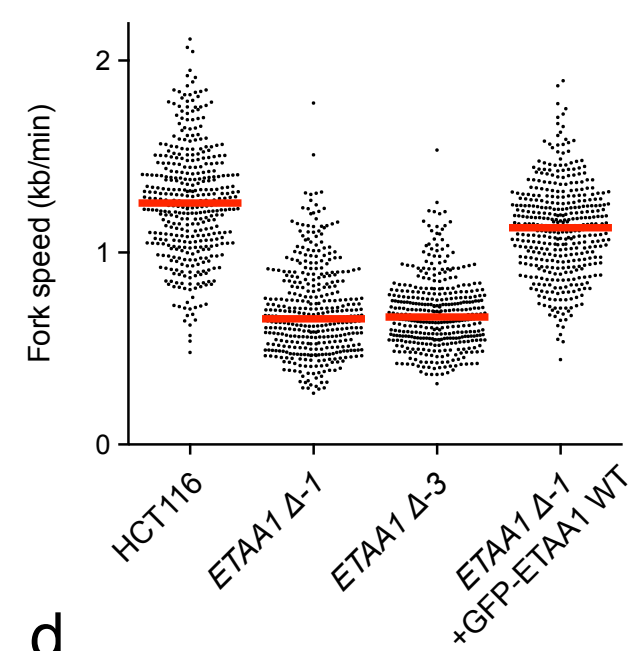

d

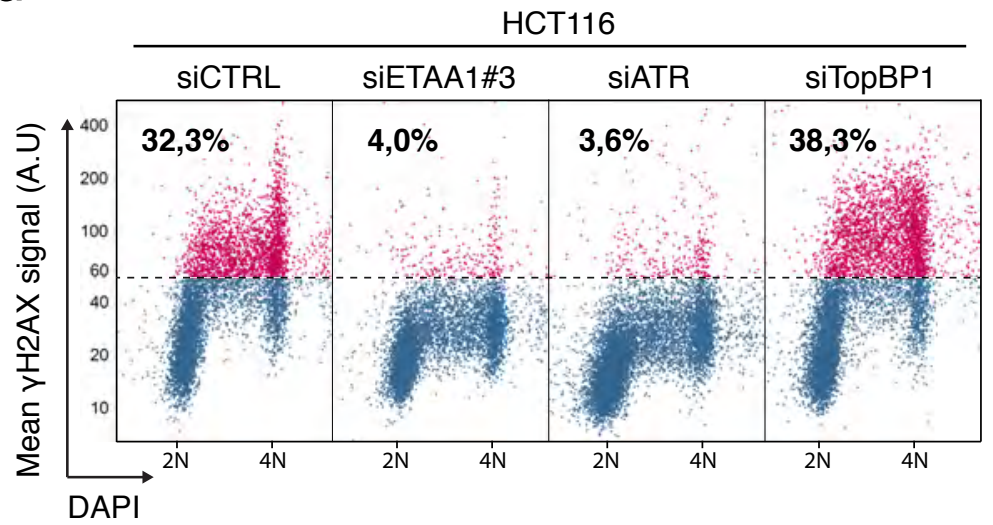

Origin

\section{b}

HCT116
ETAA1

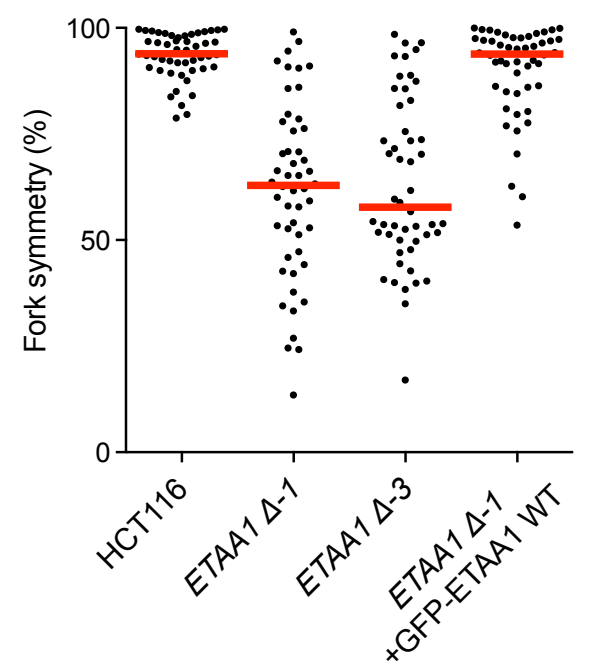

C

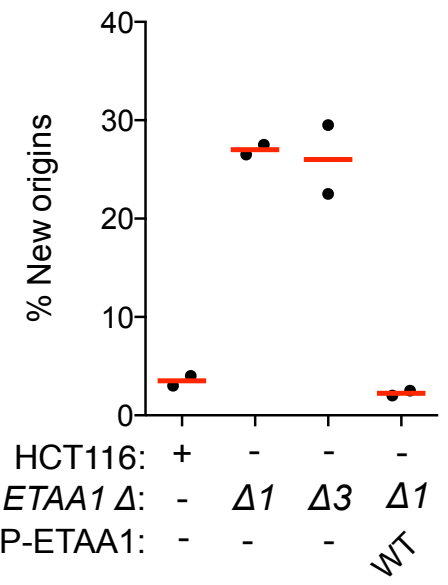

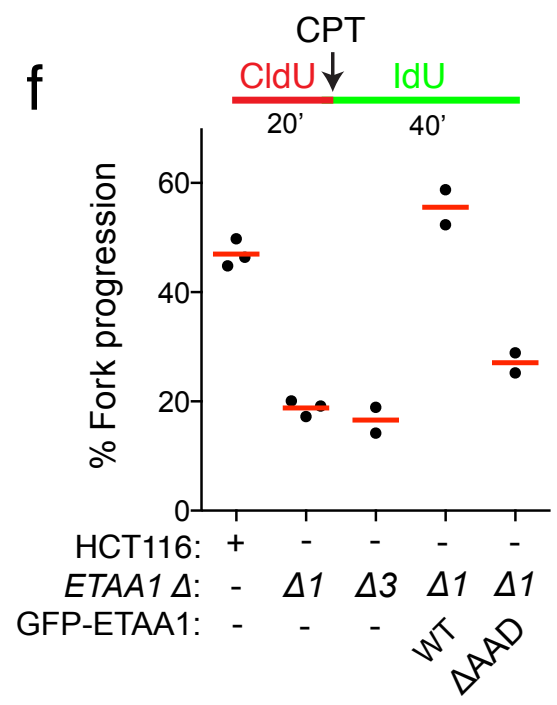

ETAA1 $\Delta:-\quad \Delta 1 \quad \Delta 3 \quad \Delta 1 \Delta 1$

GFP-ETAA1:

CPT

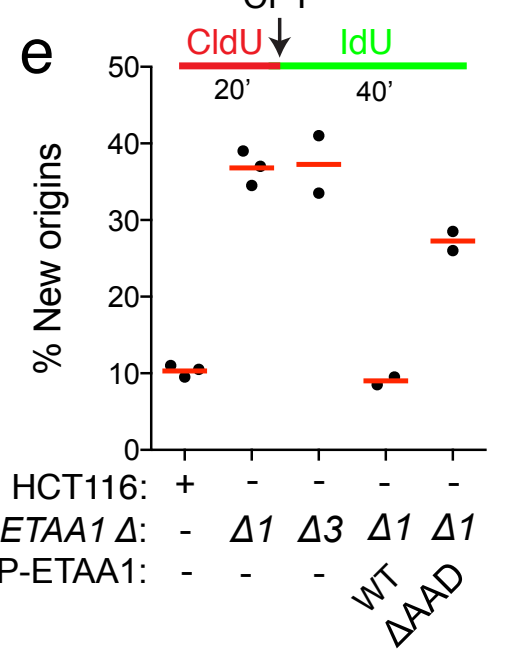

9
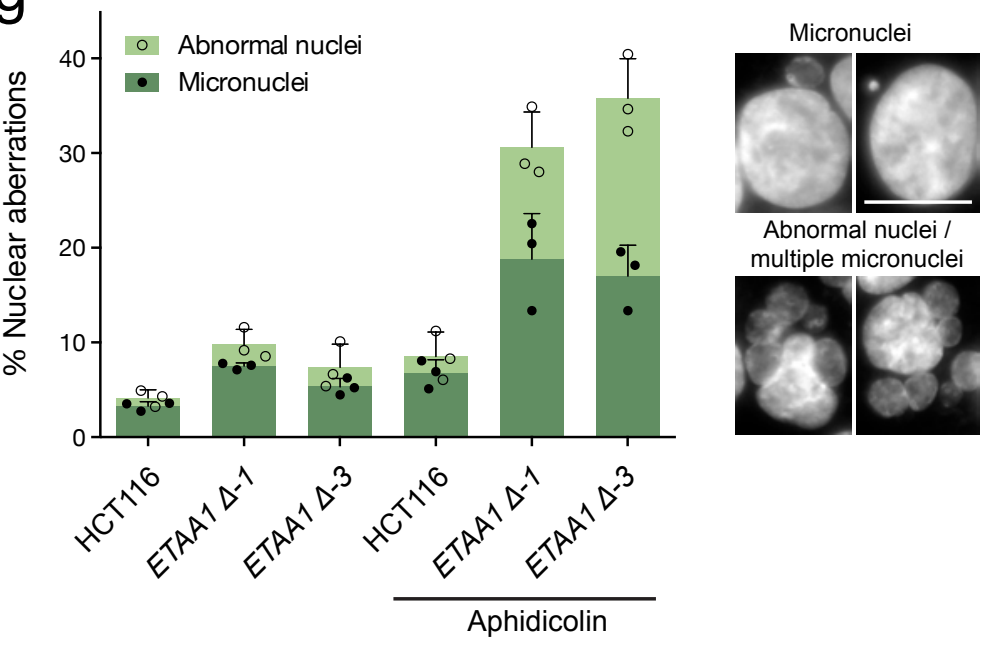

h

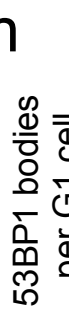
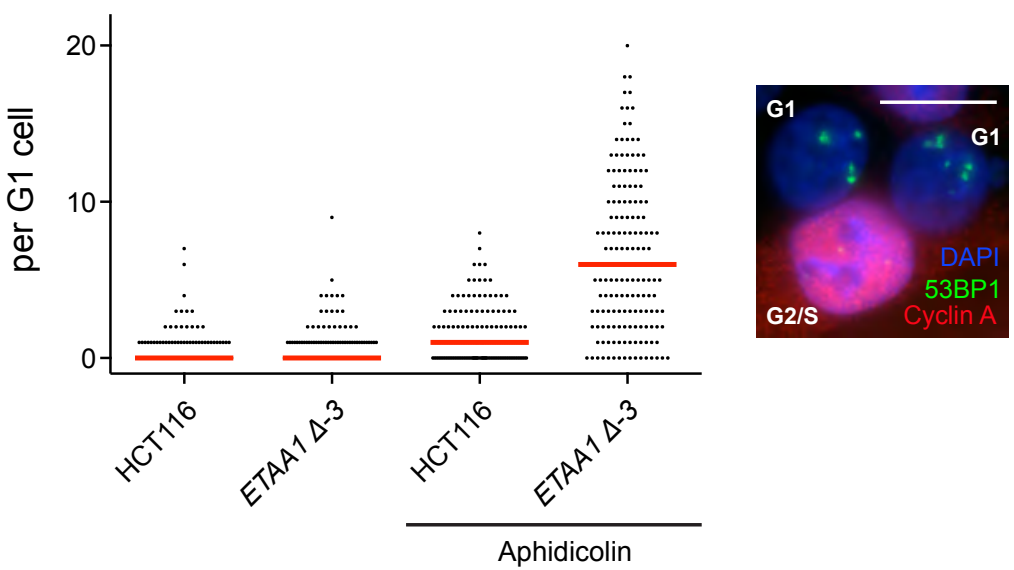


\section{Haahr et al. Figure 6}

a

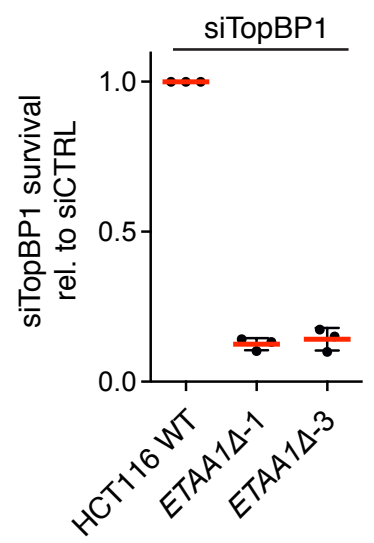

b

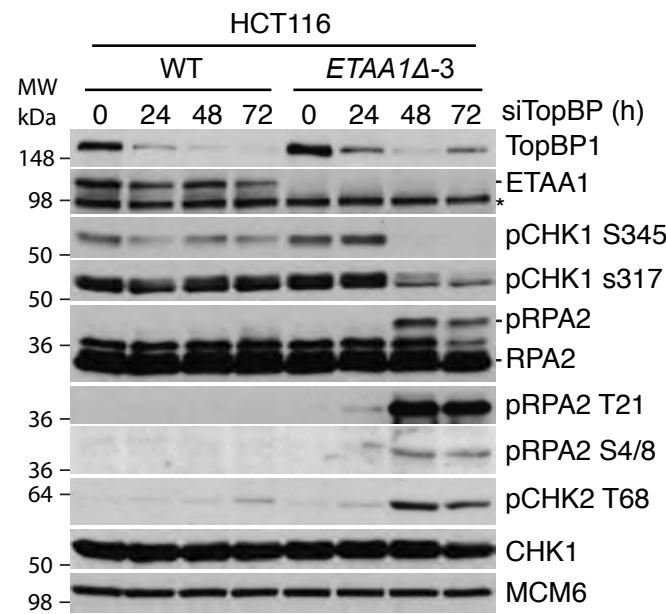

$d$

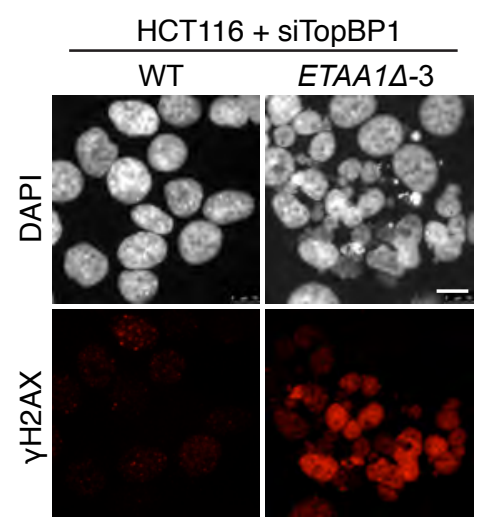

C

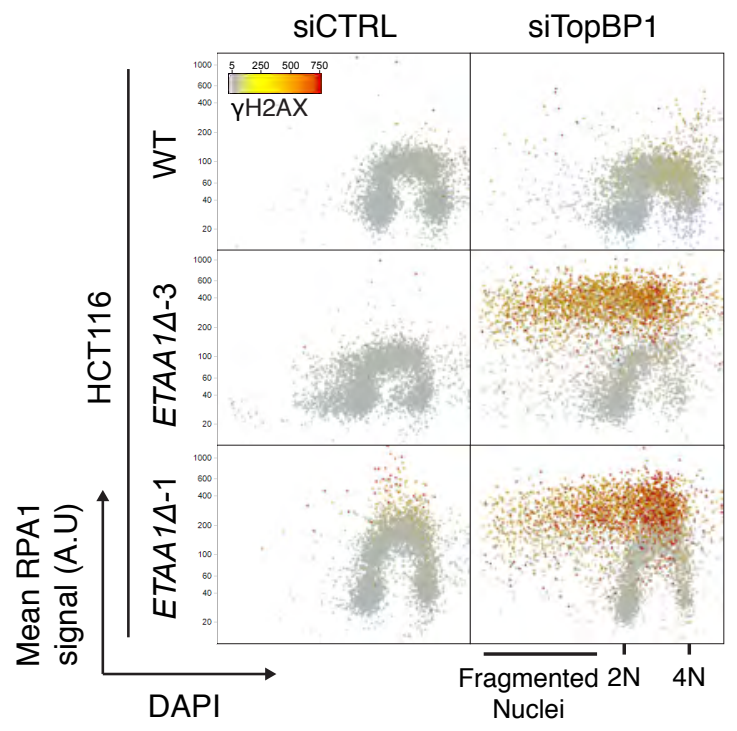

g

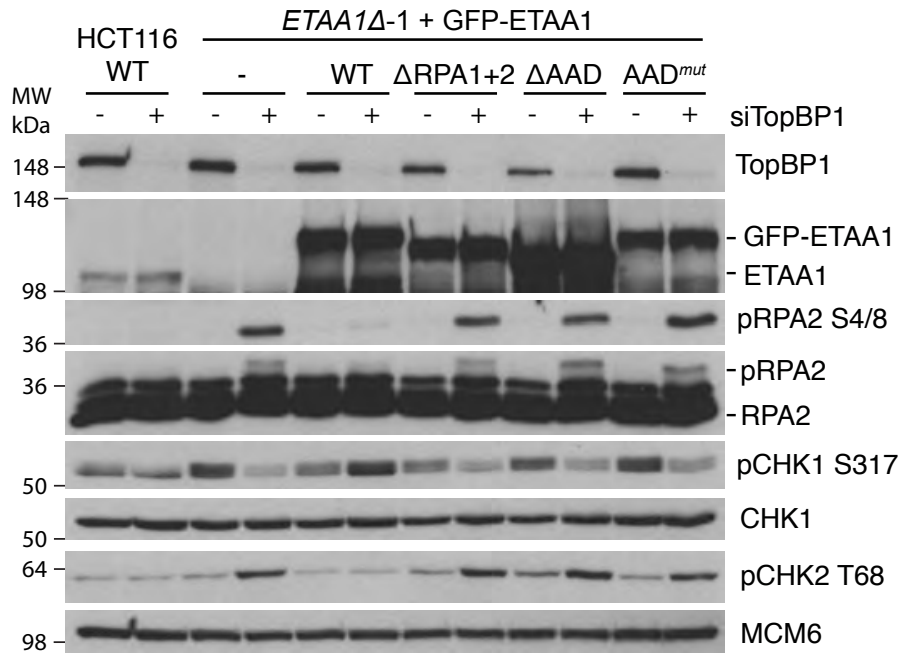

e
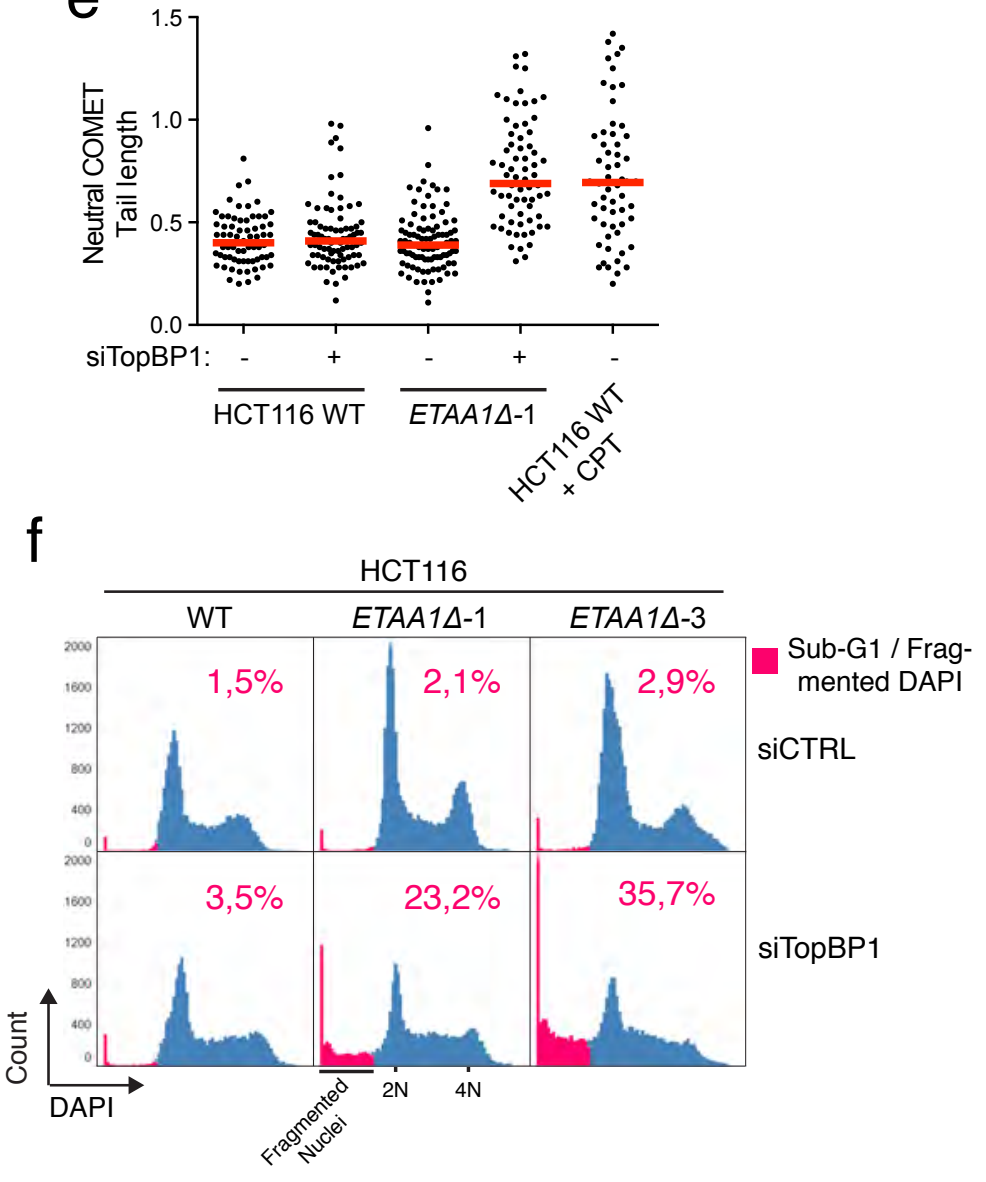

h

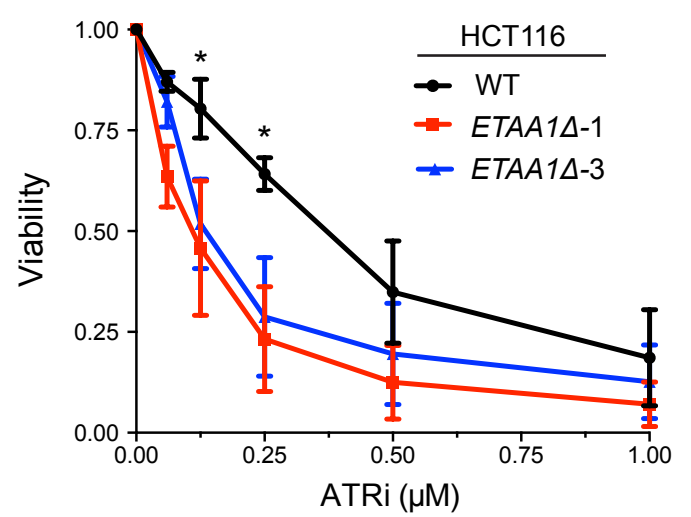




\section{Haahr et al. Figure 7}

a

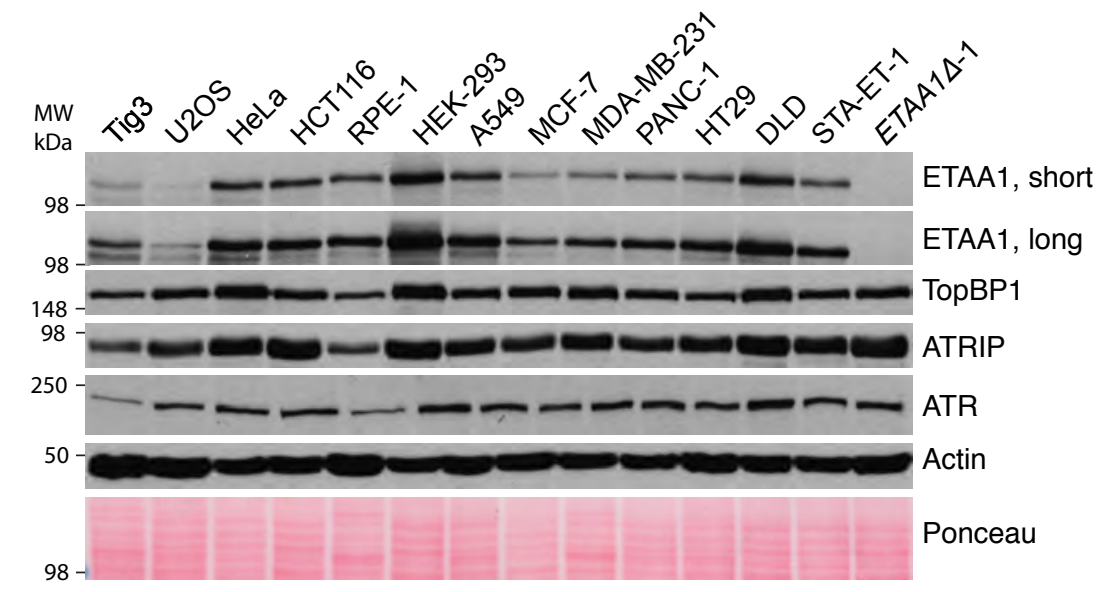

b
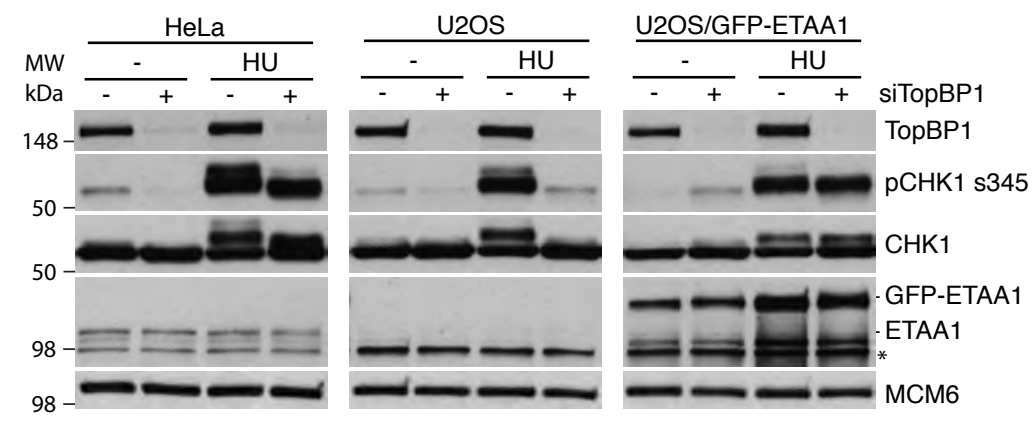

C

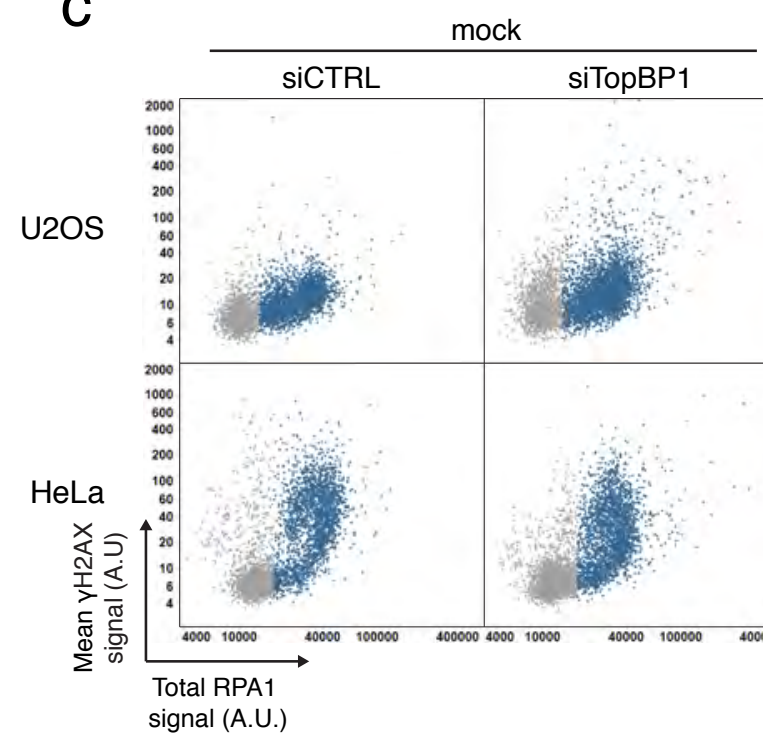

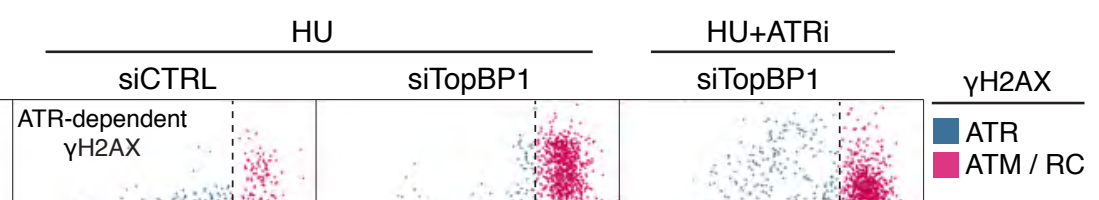
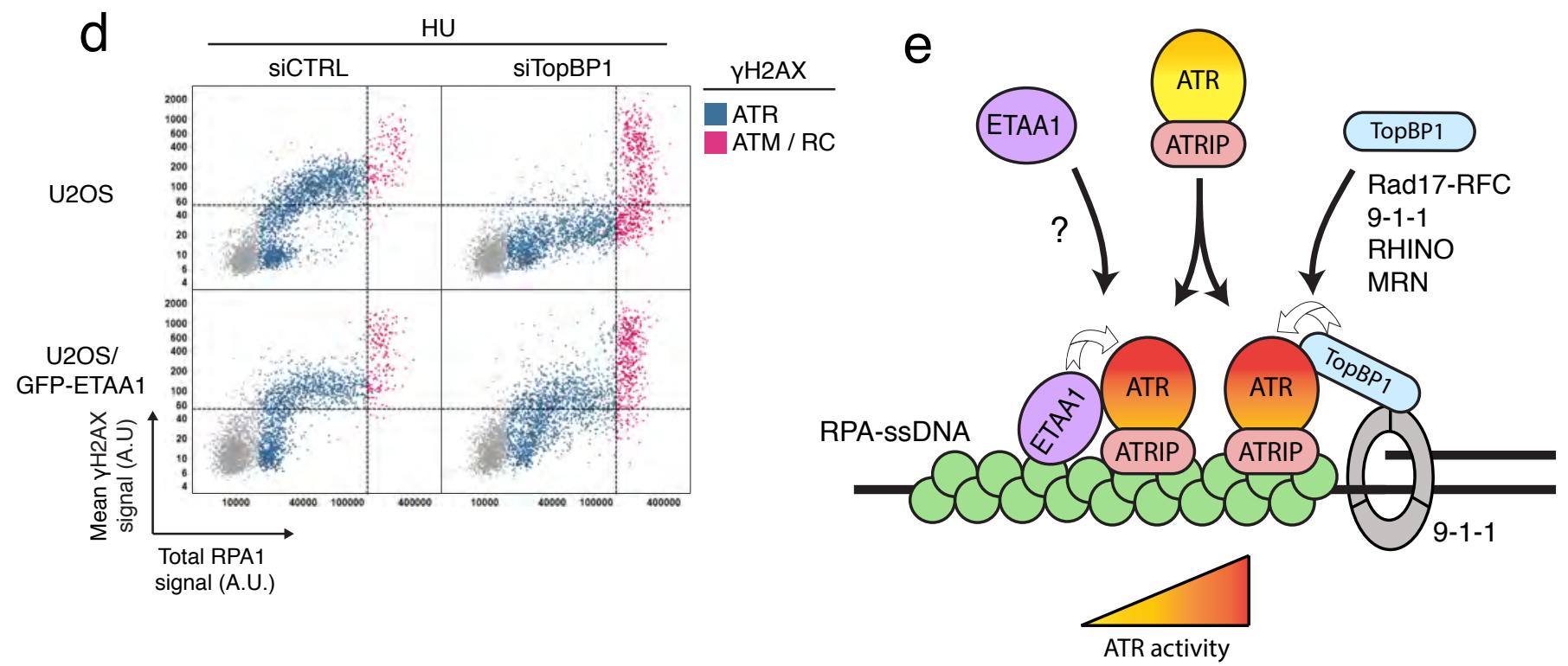
a

$>$ xIEtaa 1

MFKQRAGAAGCVKKVGSVPREAGLSGESSSYKKTPKRLSRSKHSSPAFSSPSNDVDQQHEII WDPYSPTAFKIENGRRKRPNANKCTVEISDIVNRIAPKNEKPADAAYLQMWIGDDAIPSTPVVA RSRSKASLRPRSLHTEEELMKLARQFDRNLIEAIQPQEQRDLSADEKTVPLGTAQIYETDTFLED IPEEDVELALKSVSQSSGISTSSHGQKAVDQDAEAALNALFDCSTQKVSGRLSQTLSDISIGSTH GLHVSVK GNTGEVSLSEKSDGNK $\overline{F E T P T V S K R N T S H S E S N S N V L H T S K ~} \overline{R D G S I L P K E K S R Q T E ~}$ SHGVSDPAAAISNSQDDFEDDWGNDLFEDDSFVMEITQNPNLIATPKTERTNSKSEQSGLYSV GSKSFENNKCNDKSVVPNKANHFKFVSRTSNVPVDSHSVGK RDNNISVPSICNVSQLPLNSQN NNSVQNRRMQSNSSLVPEKDAFTRGLSKNHSTNAEMLGLLNVPAKPSHCHVPQK $\overline{\text { NHAPIPE }}$ KASVQLDEWDDPKFSDDVLDMFCKSDSLWETNEDDDDLLYQVCDDVERLTQAQISNEGVNK MENTQVTSLSGAKTVPGITKQVQPSQHFGQNKNGSQIRQFSSSATNKFSGNATRYNSSTNSSL QKITAPSAGTGLRVCGNAFTFNENGGLSKTNPAPVKFGRFNSVPSASECTKTLFIGTQSQSMG NPQAATTSAKSSMAPSKFTFTRTKSSPIVPSHTCTGGTSDFKYMDSQEVAGNKNHKNQLVPKN ELLNQPFNLKRQLSDSVLQSTKVFVSERNKKCSMEEIERKKQEALARRQKRLHVLSGETAHP

Sequence Coverage: $52.3 \%$

b

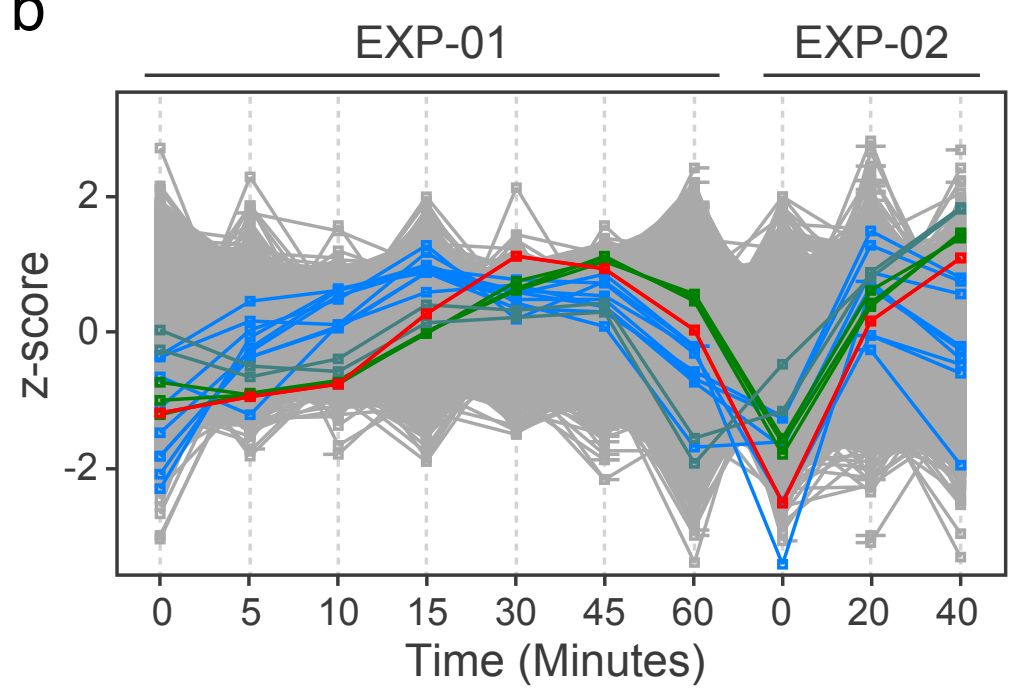

- Etaa1

- RPA1-3

- ATR/ATRIP

- NHEJ

d

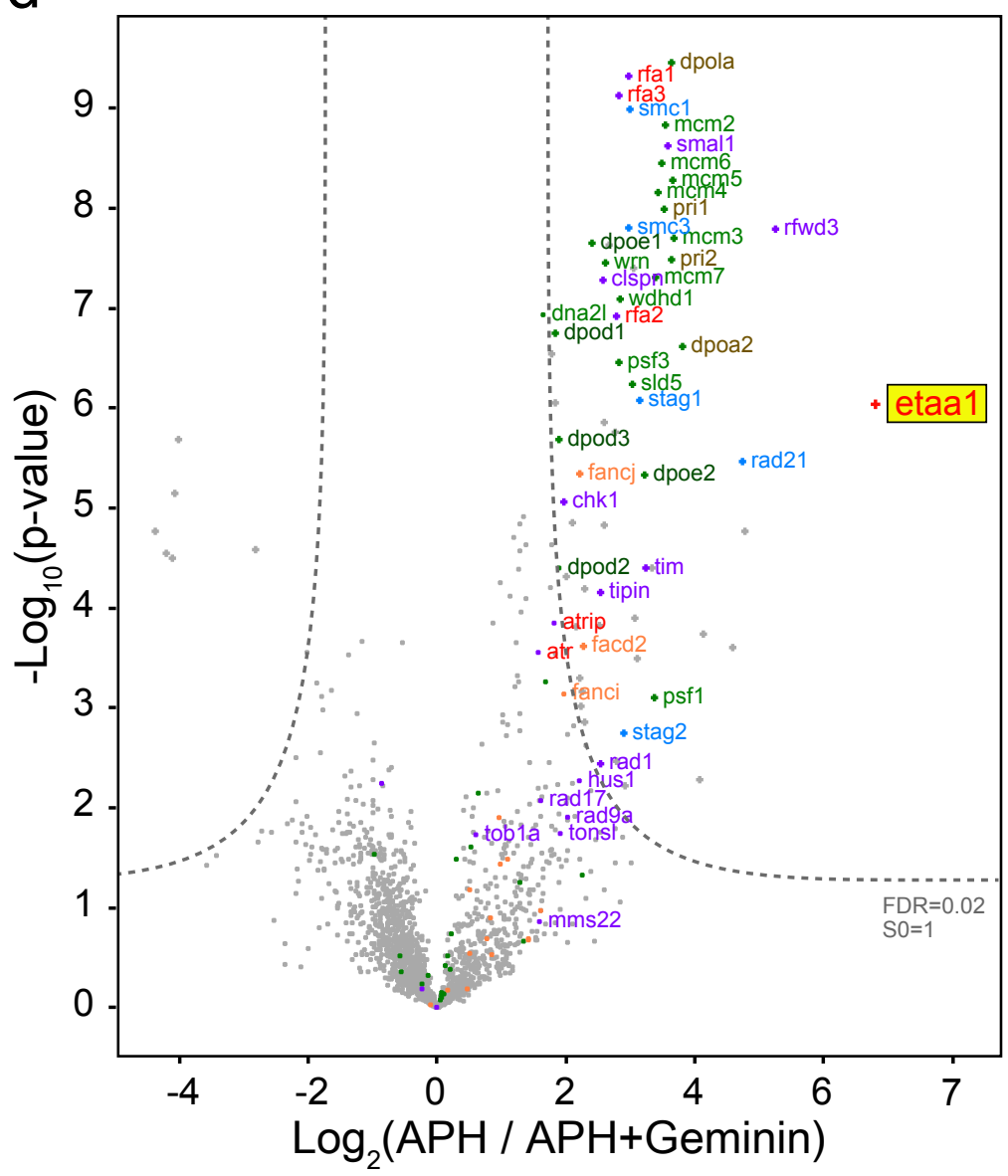

C

\begin{tabular}{|c|c|c|}
\hline \multicolumn{3}{|c|}{ Rank Distance Protein } \\
\hline 1 & 0.0006 & rfa1 \\
\hline 2 & 0.0051 & rfa2 \\
\hline 3 & 0.0053 & rfa3 \\
\hline 4 & 0.0120 & dpoa2 \\
\hline 5 & 0.0363 & helb \\
\hline 6 & 0.0388 & nsrp1 \\
\hline 7 & 0.0413 & rad9a \\
\hline 8 & 0.0421 & dpolq \\
\hline 9 & 0.0430 & dpola \\
\hline 10 & 0.0474 & etaa1 \\
\hline 11 & 0.0504 & pri1 \\
\hline 12 & 0.0610 & smal1 \\
\hline 13 & 0.0705 & rtel1 \\
\hline 14 & 0.0728 & com1 \\
\hline 15 & 0.0787 & pri2 \\
\hline 16 & 0.0792 & helq \\
\hline 17 & 0.0802 & rad17 \\
\hline 18 & 0.0807 & wrn \\
\hline 19 & 0.0868 & mlh1 \\
\hline 20 & 0.0971 & fancj \\
\hline 21 & 0.1026 & rad1 \\
\hline 26 & 0.1204 & dna2l \\
\hline 33 & 0.1587 & rfwd3 \\
\hline
\end{tabular}

Total: 2668 Profiles

\section{Unperturbed Replication}

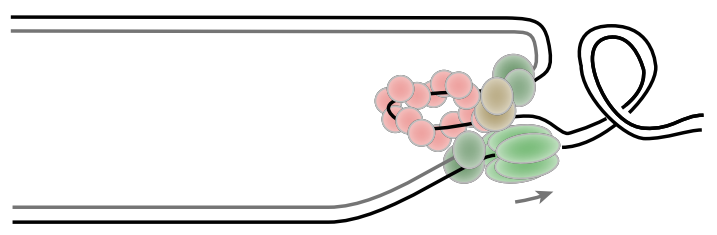

\section{Helicase Uncoupling}

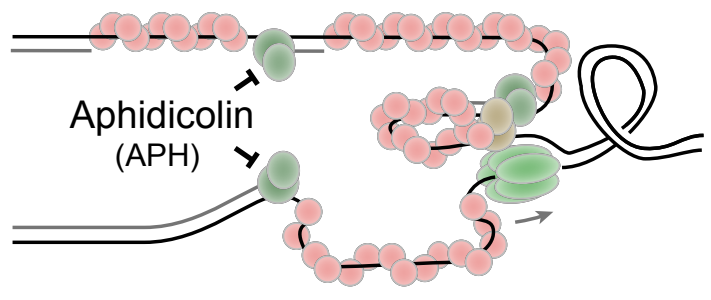




\section{Haahr et al. Figure S2}

a

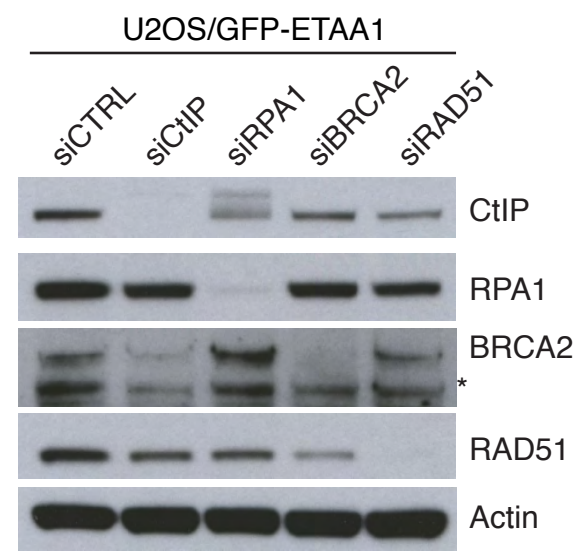

b

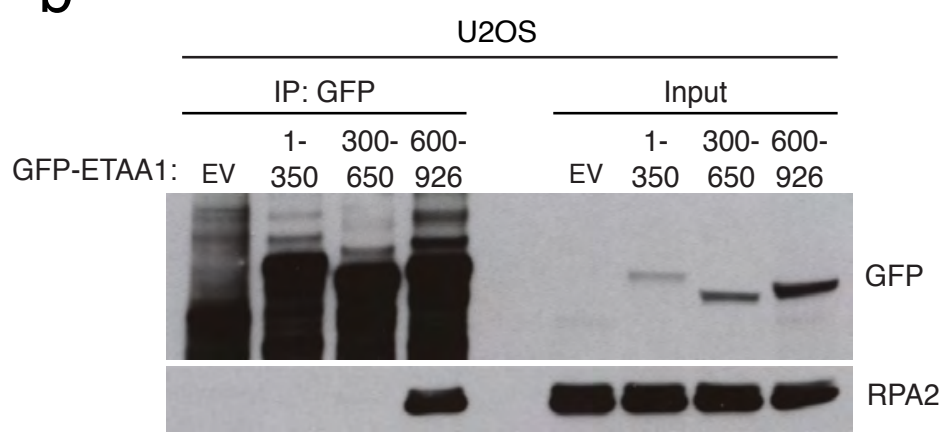

C

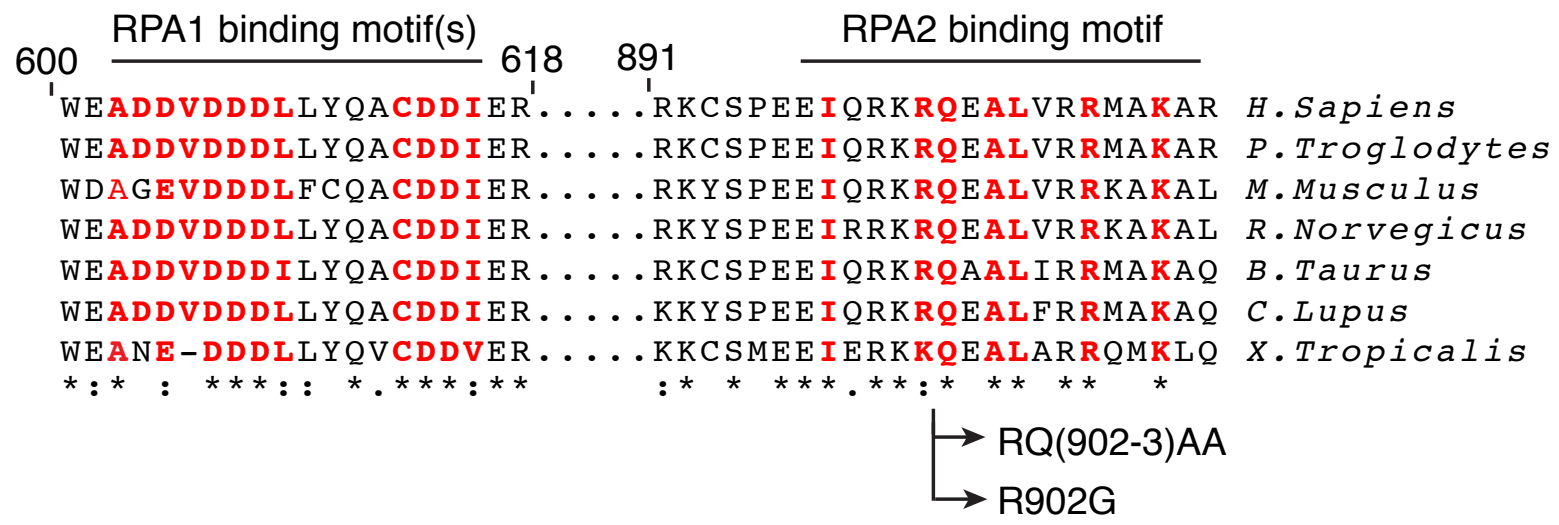

d

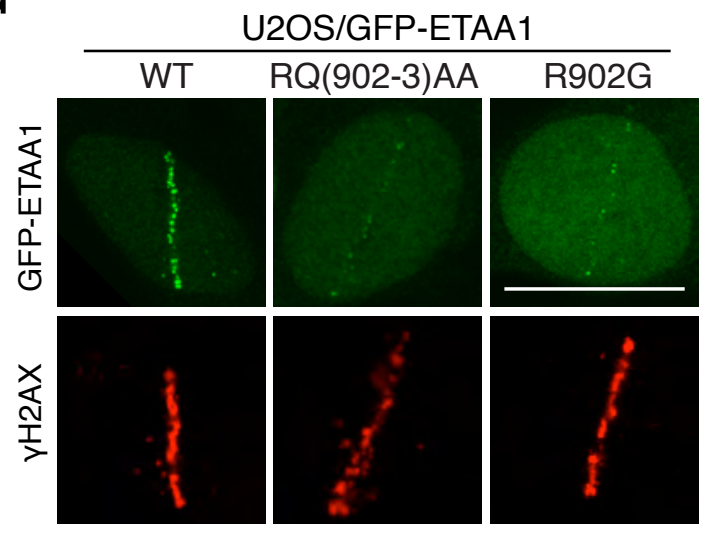

e

f

U2OS/GFP-ETAA1

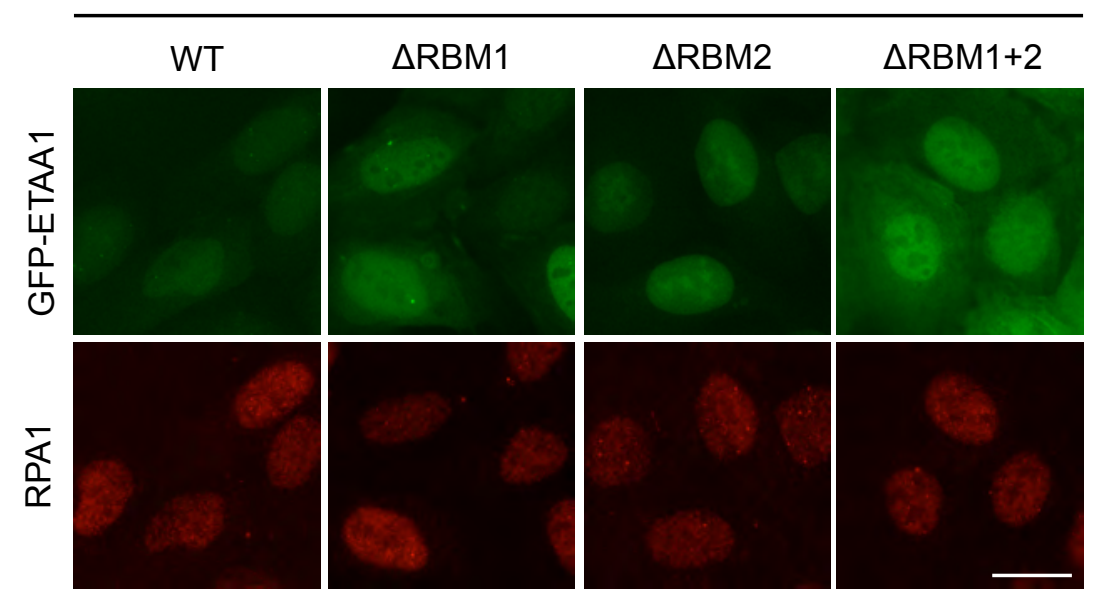




\section{Haahr et al. Figure S3}

$\begin{array}{cccc}\text { ATR Activating } & \text { RPA1 binding } & \text { RPA2 binding } \\ \text { Domain (AAD) } & \text { motif (RBM1) } & \text { motif (RBM2) } \\ (56-220) & (603-618) & (891-913)\end{array}$

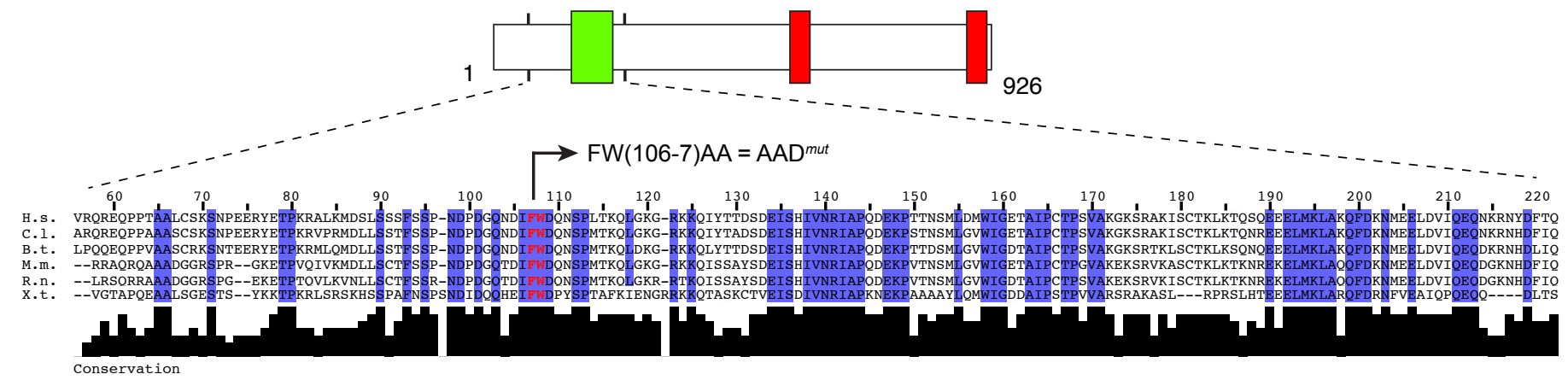

b

\begin{tabular}{|c|c|}
\hline $\begin{array}{c}\text { GFP- } \\
\text { ETAA1 }\end{array}$ & $\begin{array}{c}\text { Pan-nuclear } \\
\text { YH2AX }\end{array}$ \\
\hline Full lenght & + \\
\hline $1-350$ & + \\
\hline $300-650$ & - \\
\hline $600-926$ & - \\
\hline $92-350$ & - \\
\hline $1-220$ & + \\
\hline $1-177$ & - \\
\hline $56-220$ & + \\
\hline
\end{tabular}

Coiled coil

(183-214)
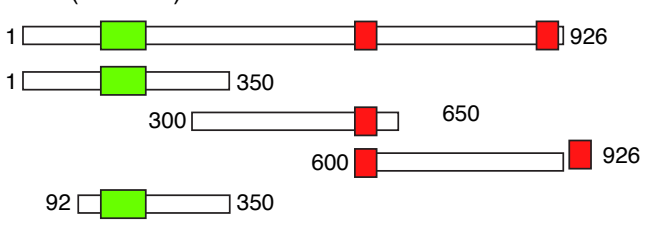

$1 \square \square 20$

$1 \square 177$

$56 \square 220$ Minimal ATR activating region
C

U2OS/

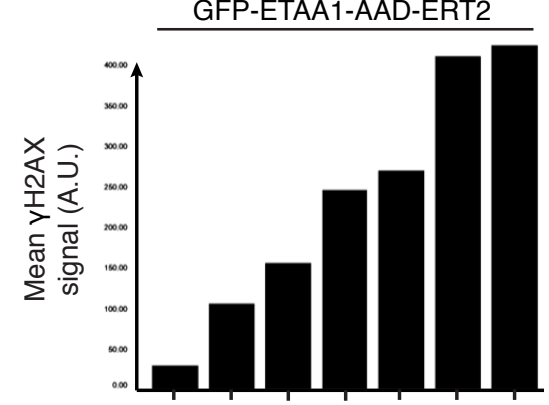

+4-OHT (min): $0 \quad 15 \quad 30 \quad 60120240480$ d
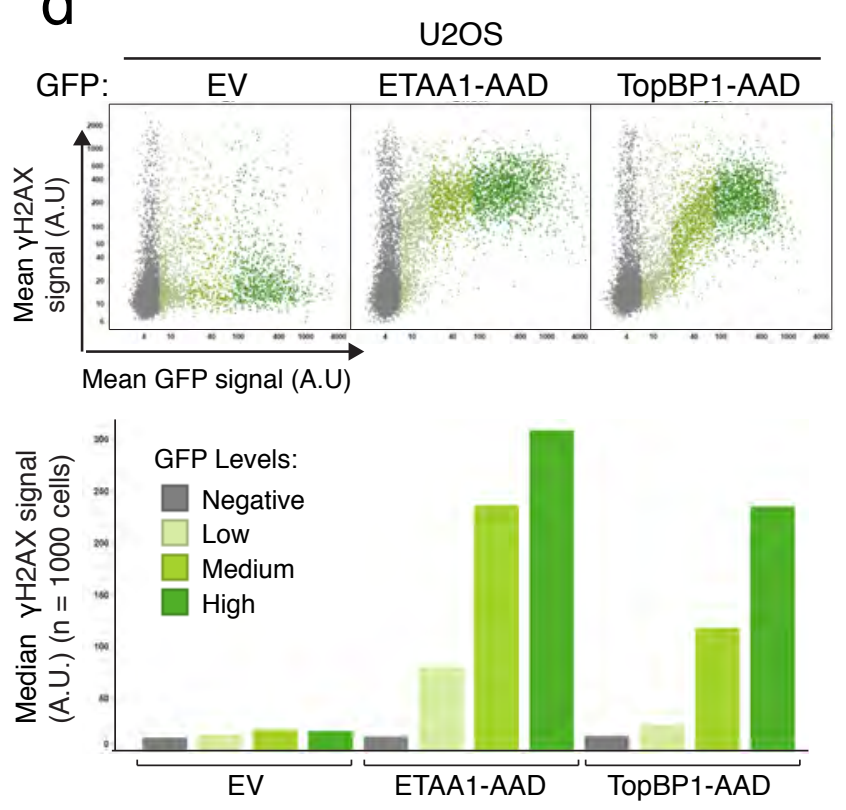

e
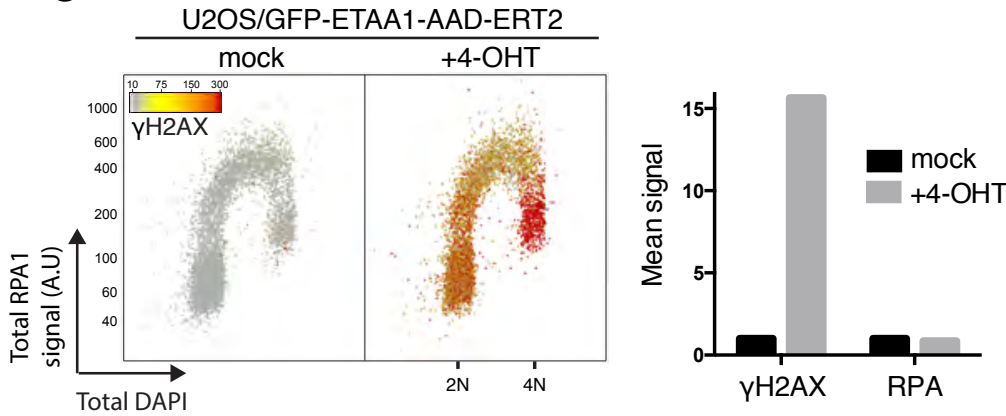

f

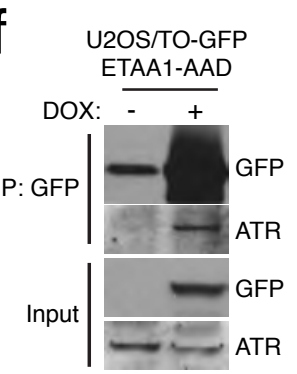

9

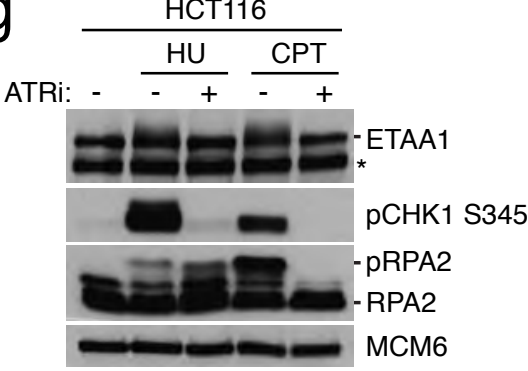

$\mathrm{h}$

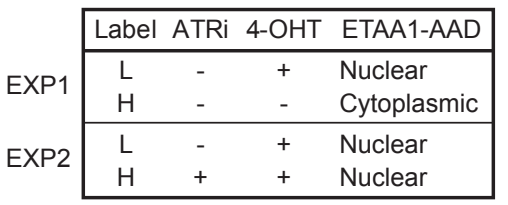

4 Biological Replicates each

$$
\text { i }
$$

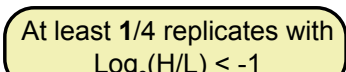

$$
\log _{2}(H / L)<-1
$$

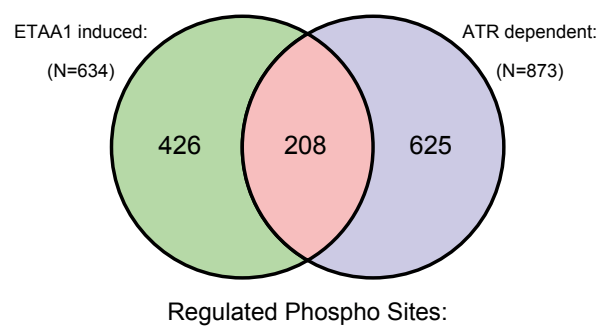

1299 / 13949

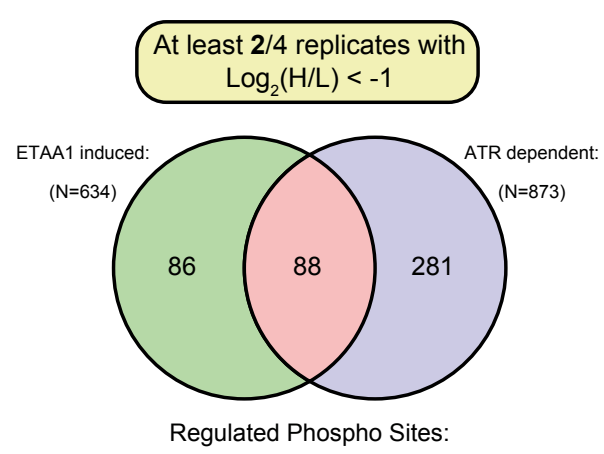

455 / 13949 


\section{Haahr et al. Figure S4}

a

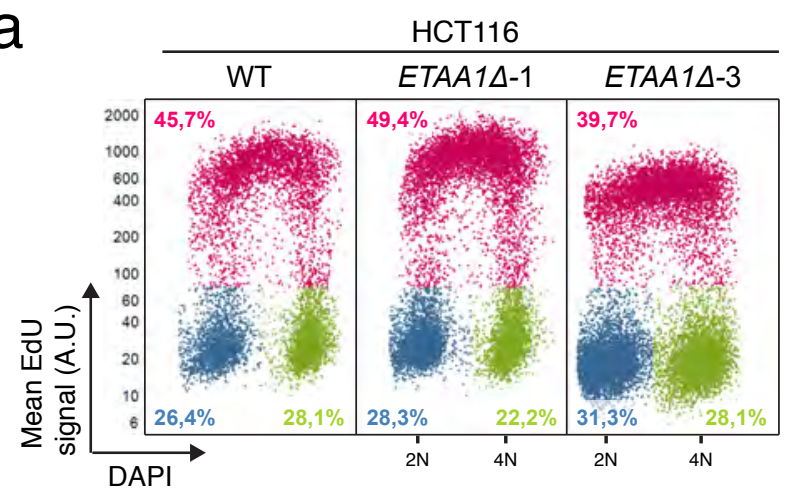

C

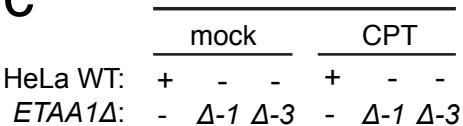

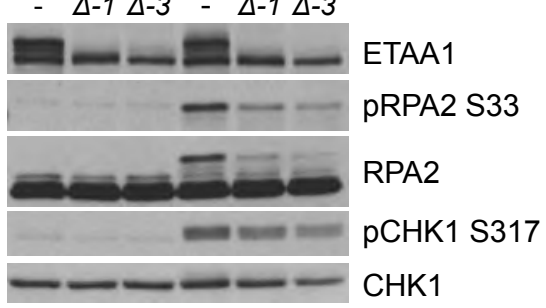

b

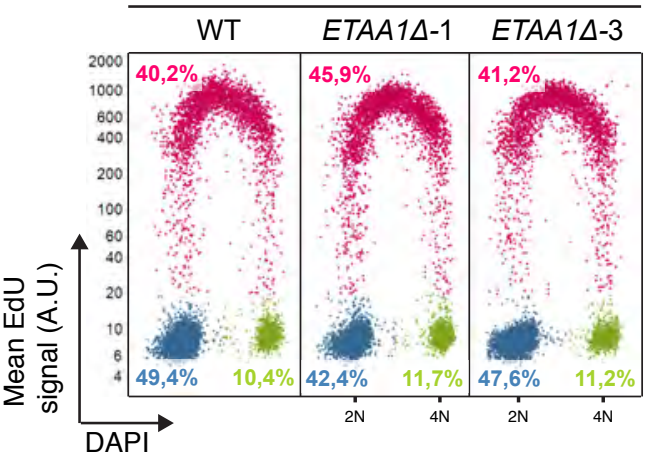

d

HU (h):

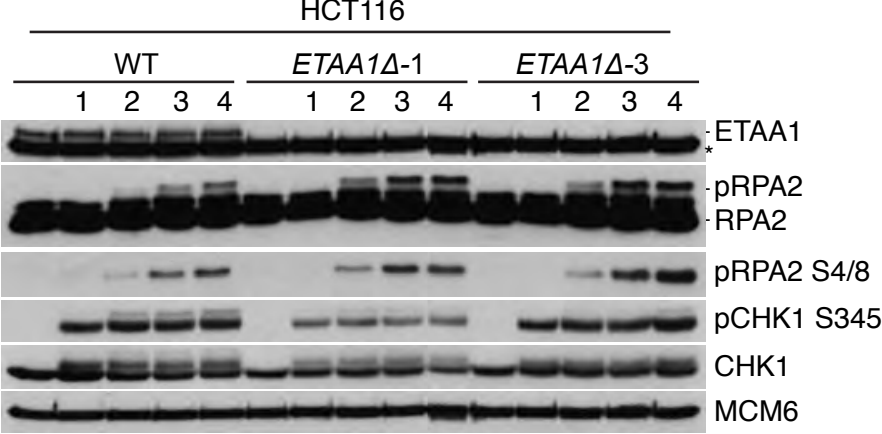

e

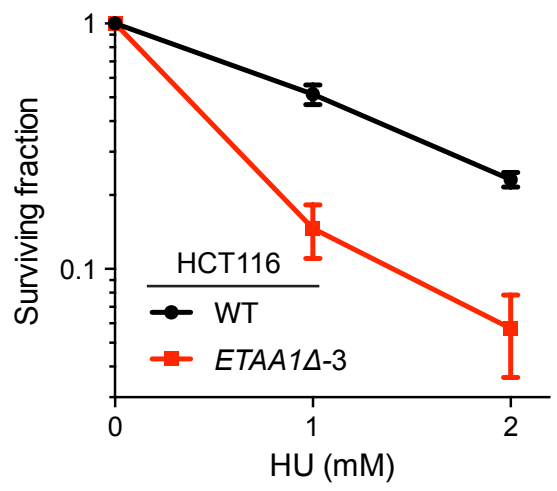

h

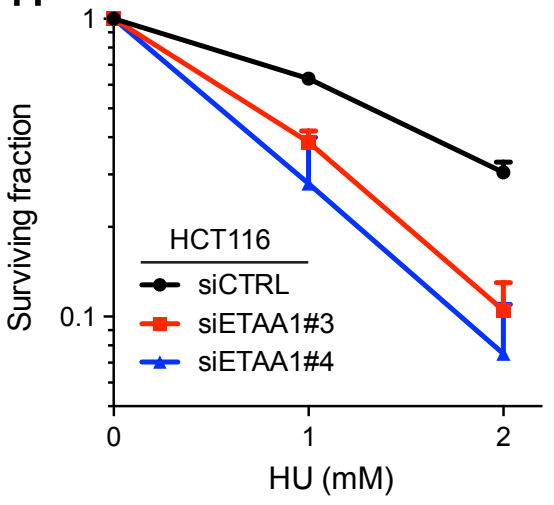

k

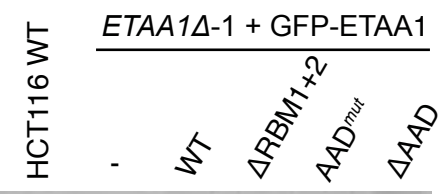

f
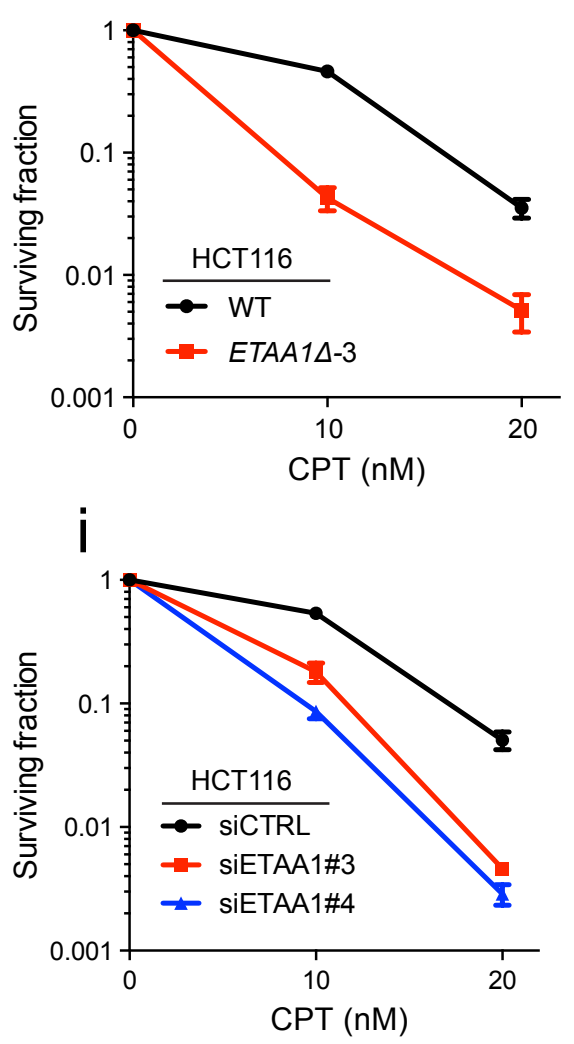

g

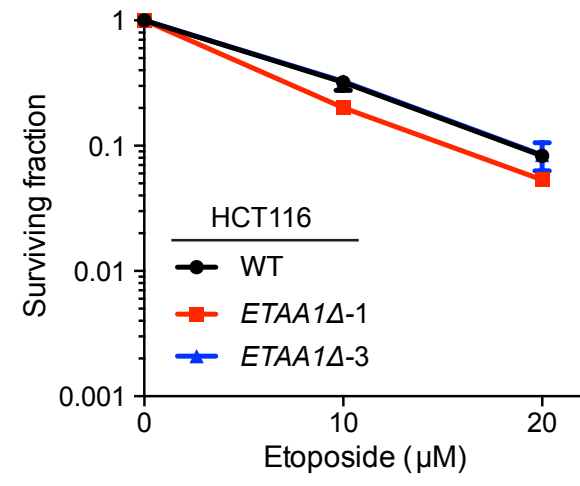

j

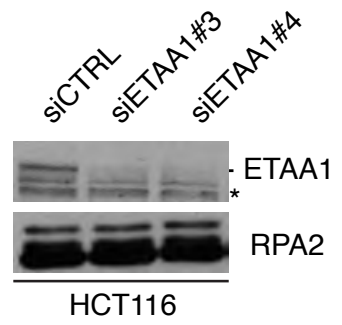

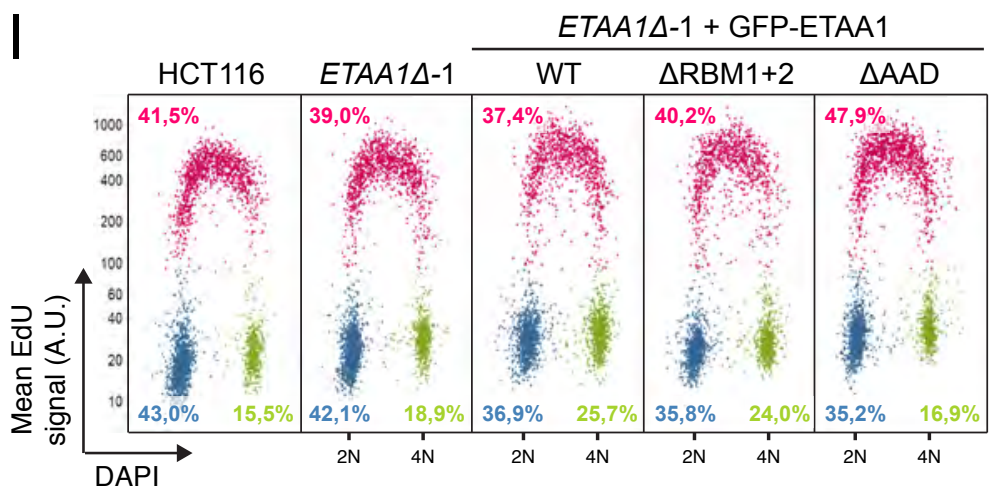



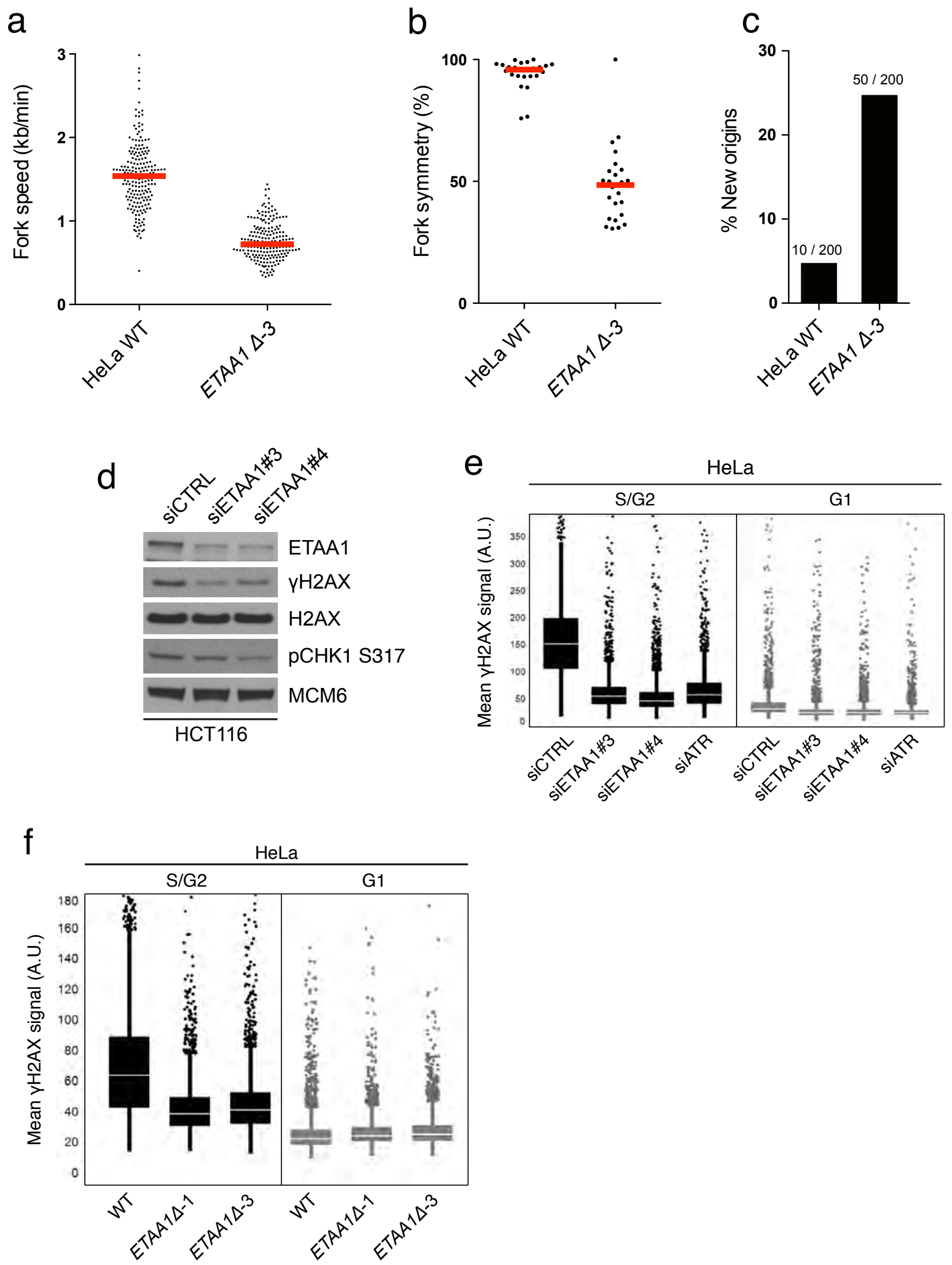


\section{Haahr et al. Figure S6}
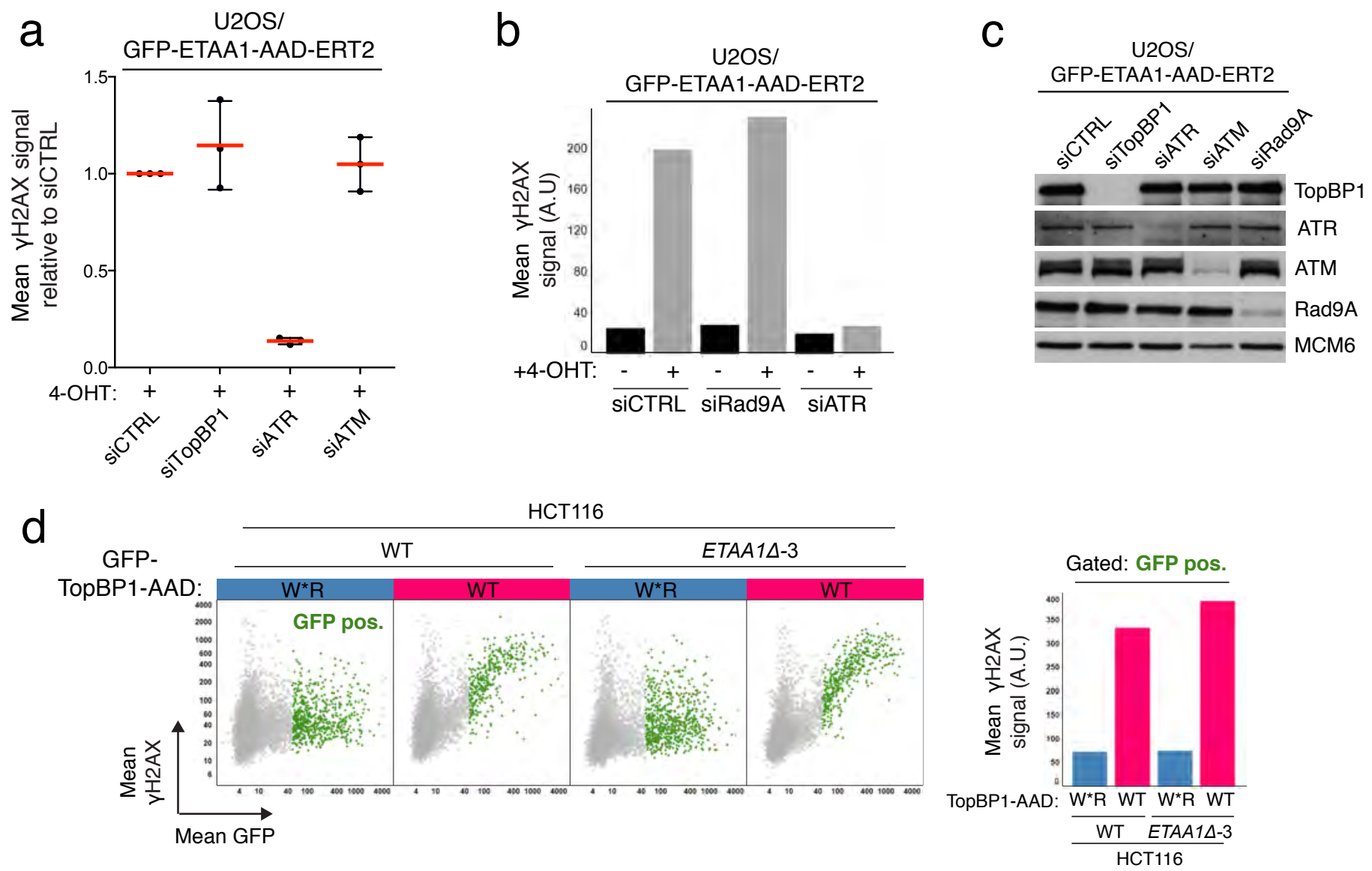

e
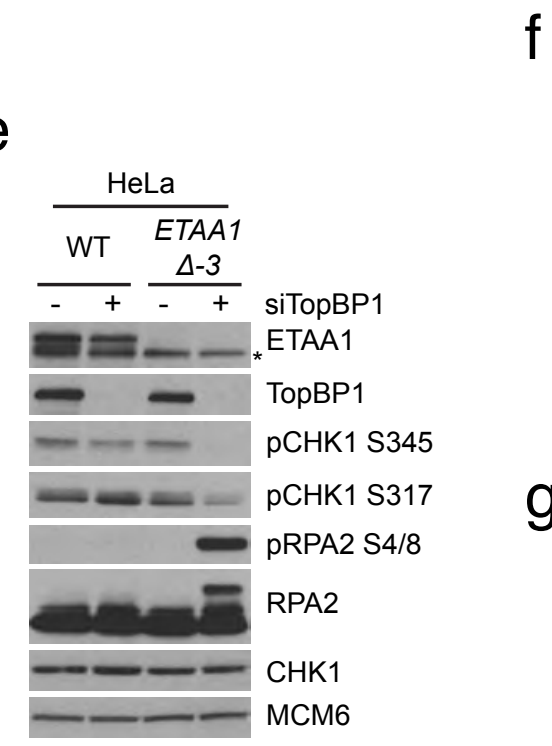

f

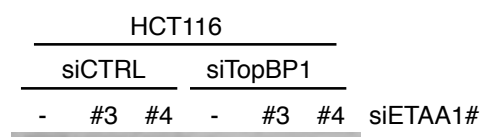

g
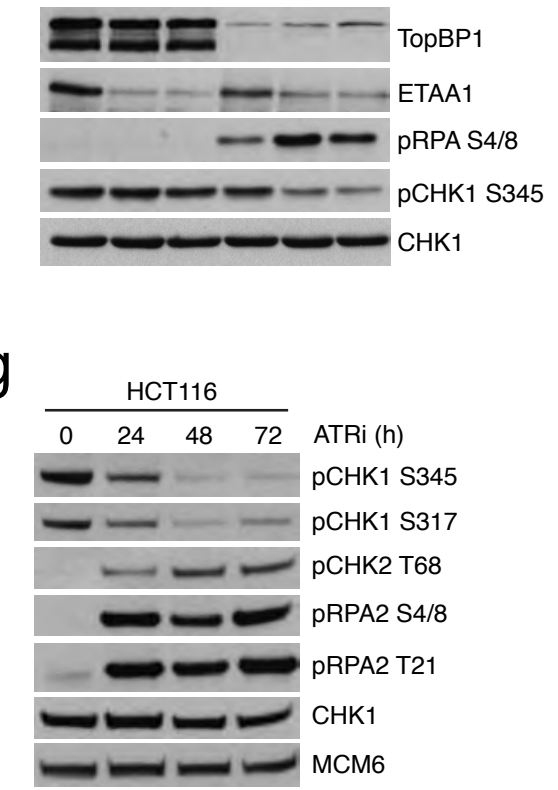

h

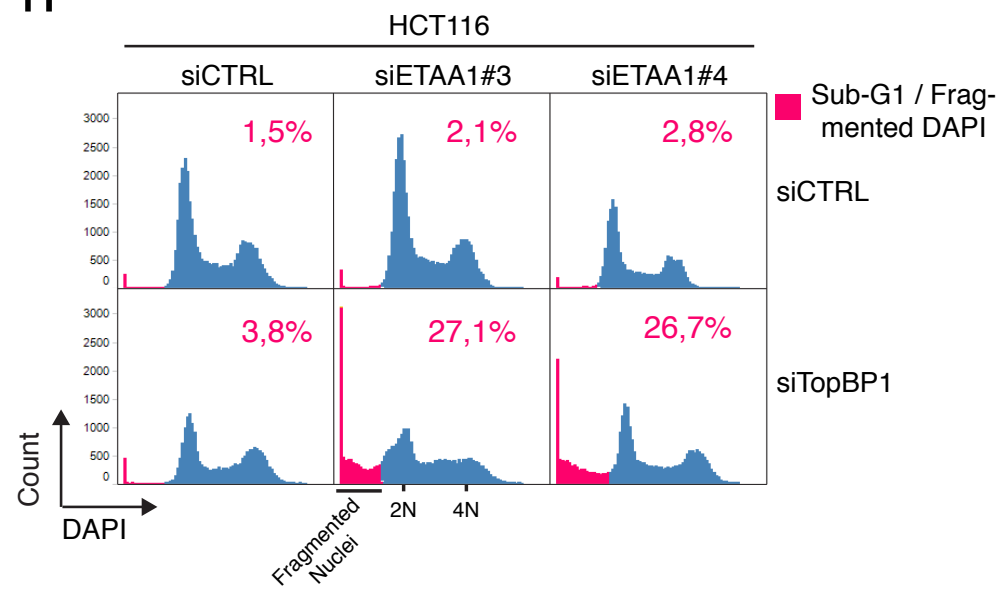

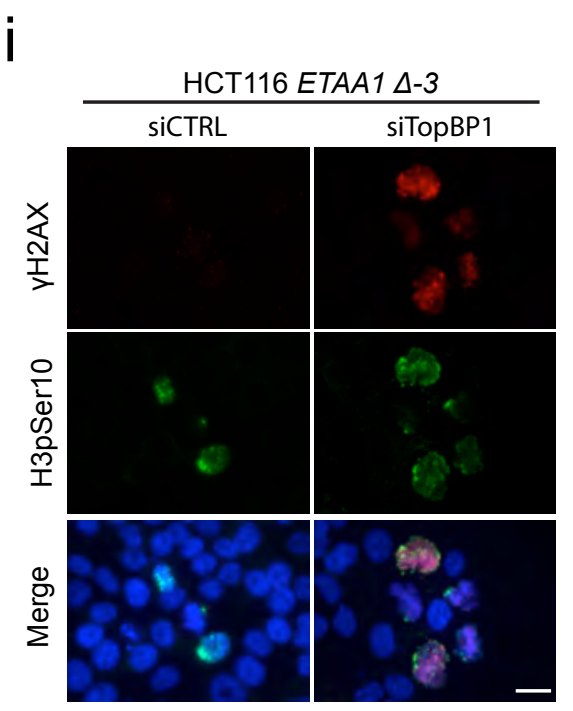

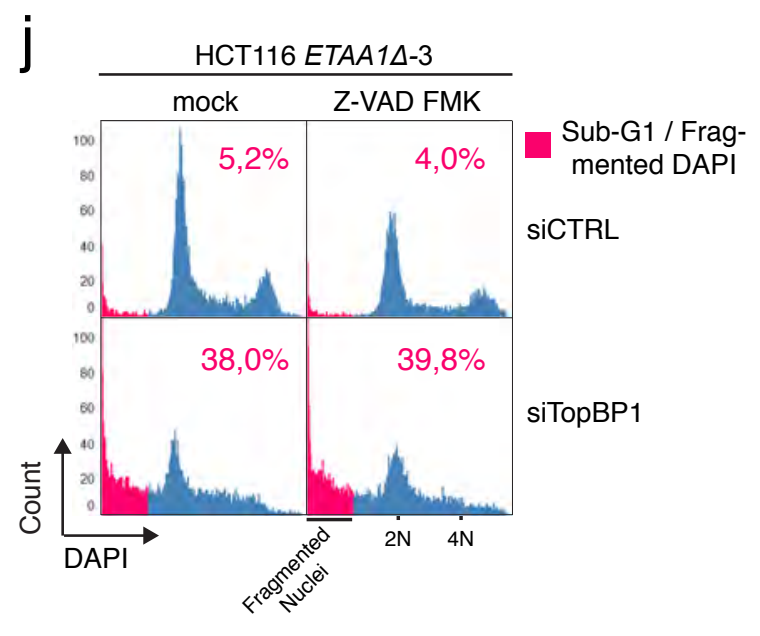



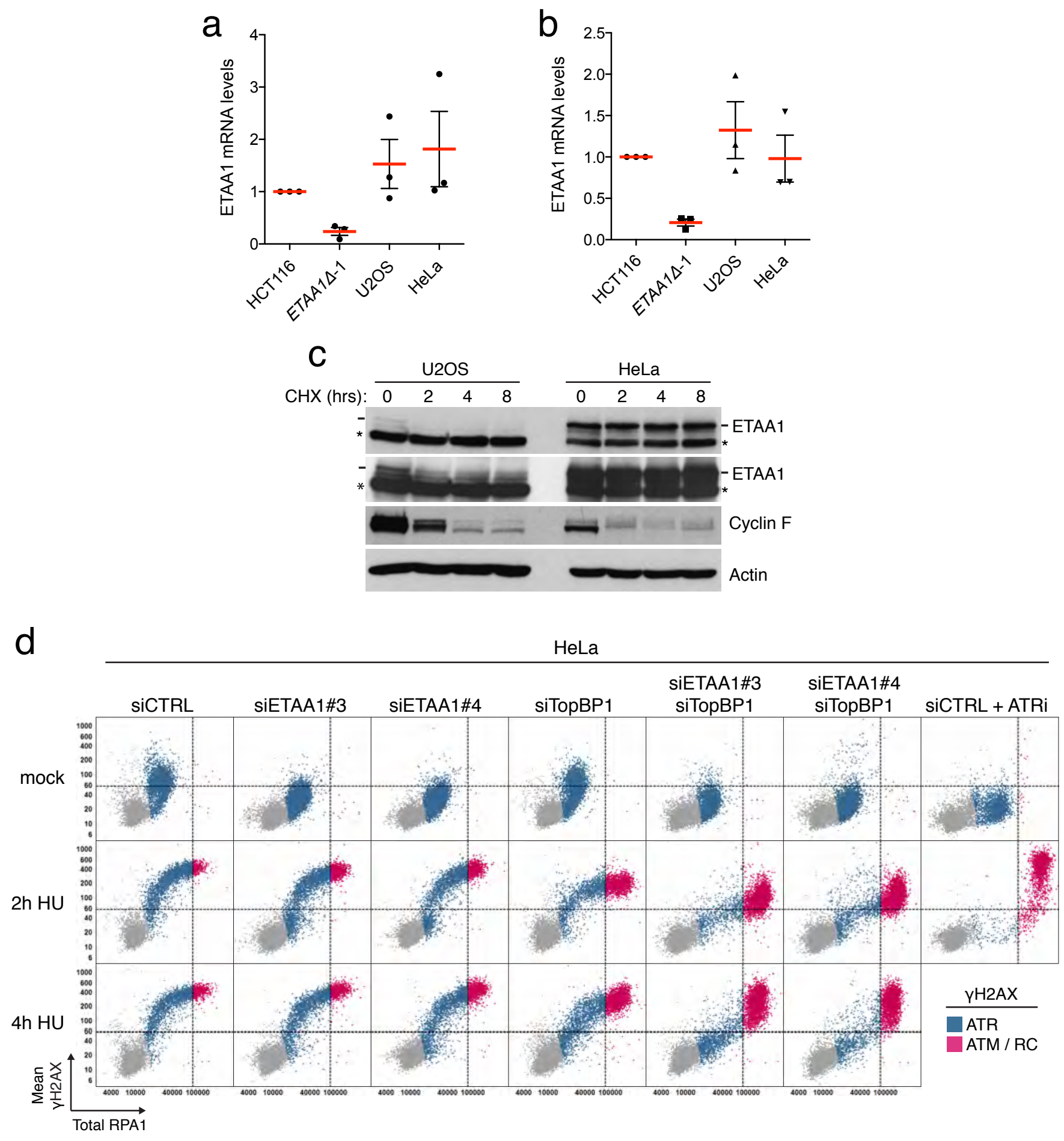

e

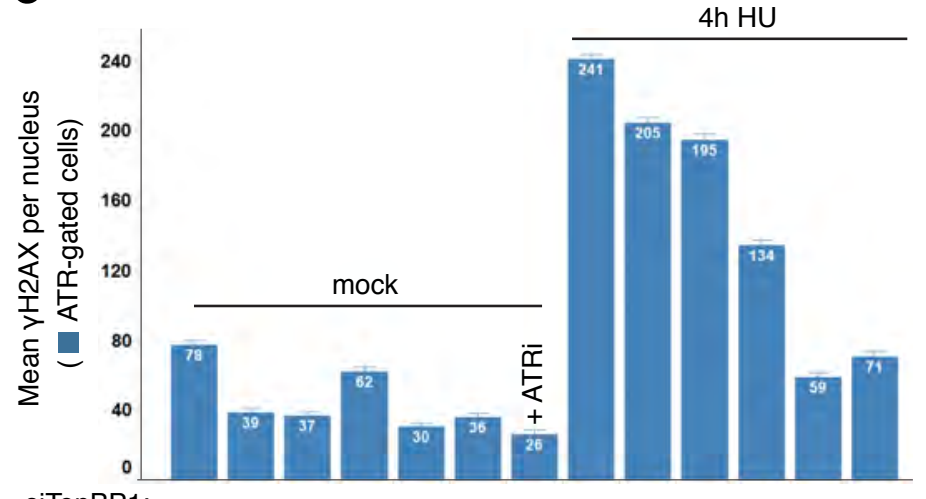

siTopBP1:

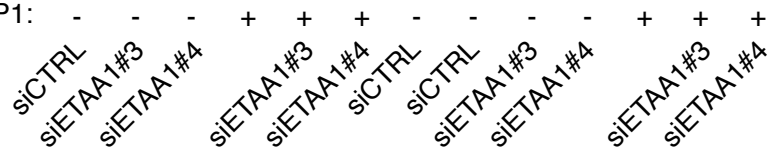

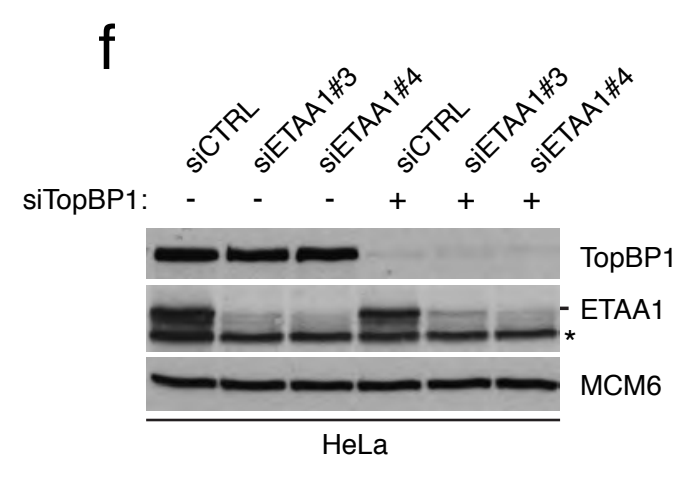


Haahr et al. Figure S8

\section{Uncropped scans with size marker $(\mathrm{kDa})$ indications}

Figure 2a

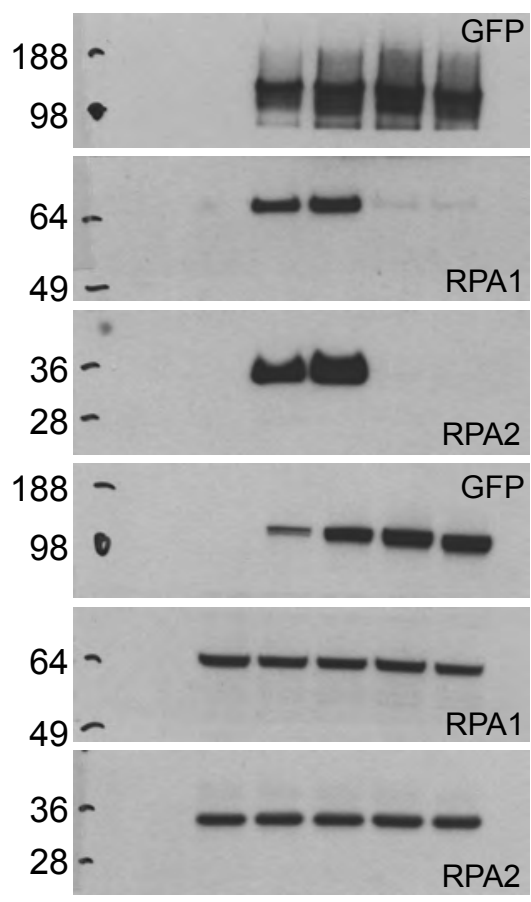

Figure $2 \mathrm{c}$

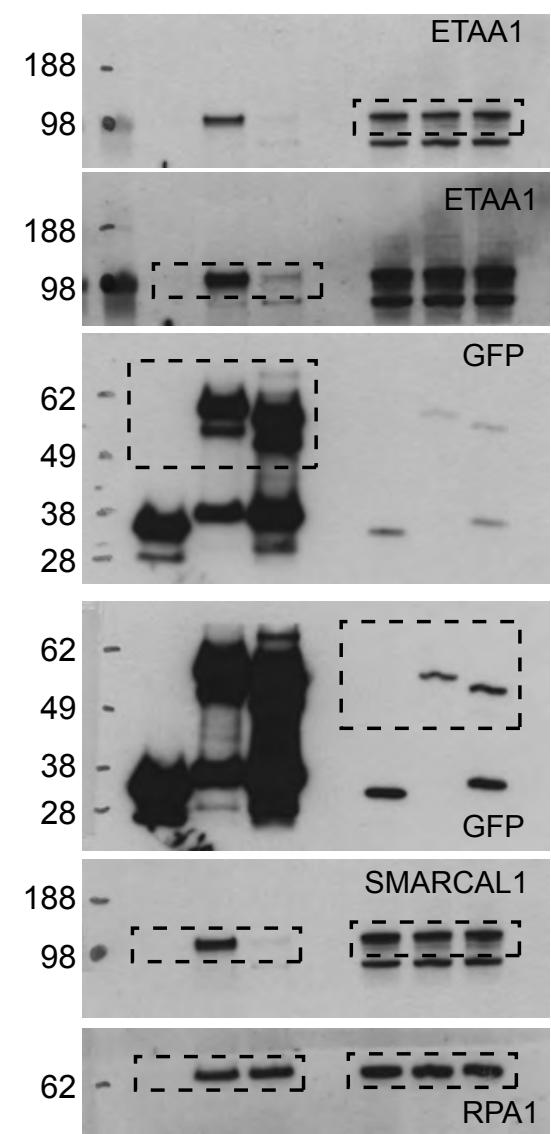

Figure $3 g$

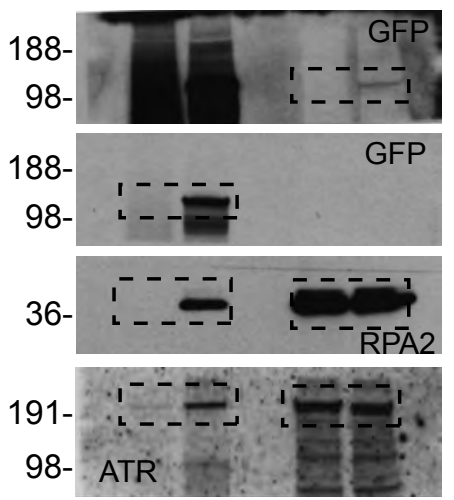

Figure $3 \mathrm{j}$

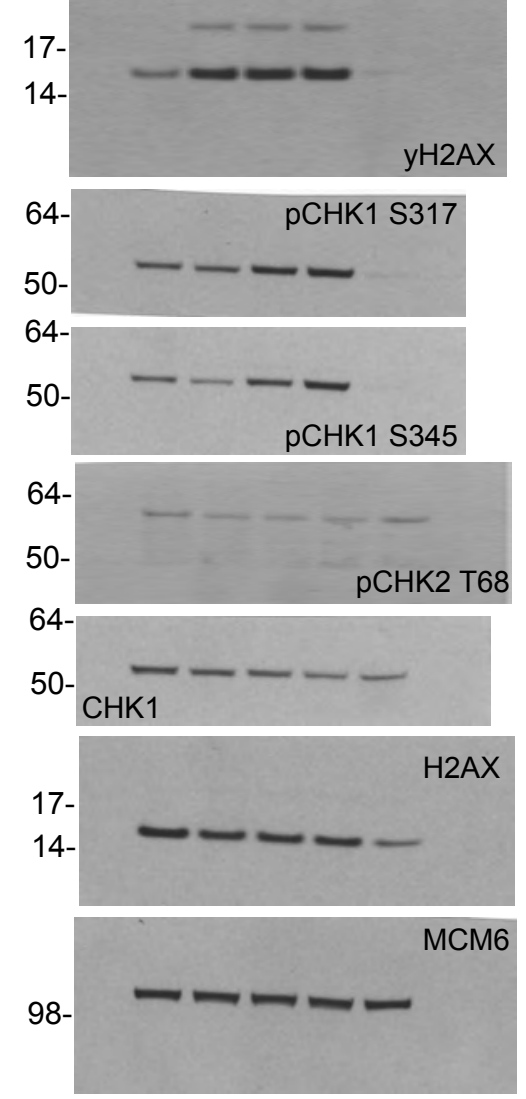

Figure $3 \mathrm{~h}$
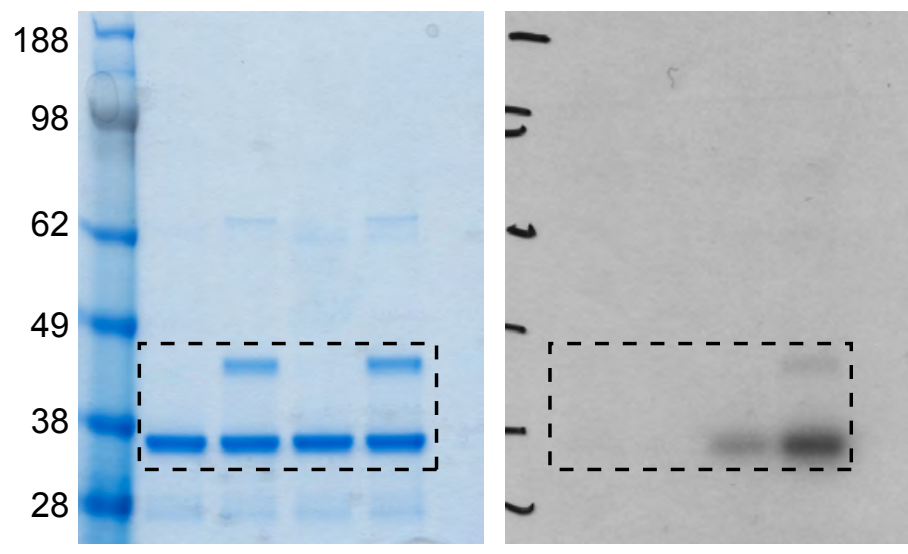

Figure $4 a$

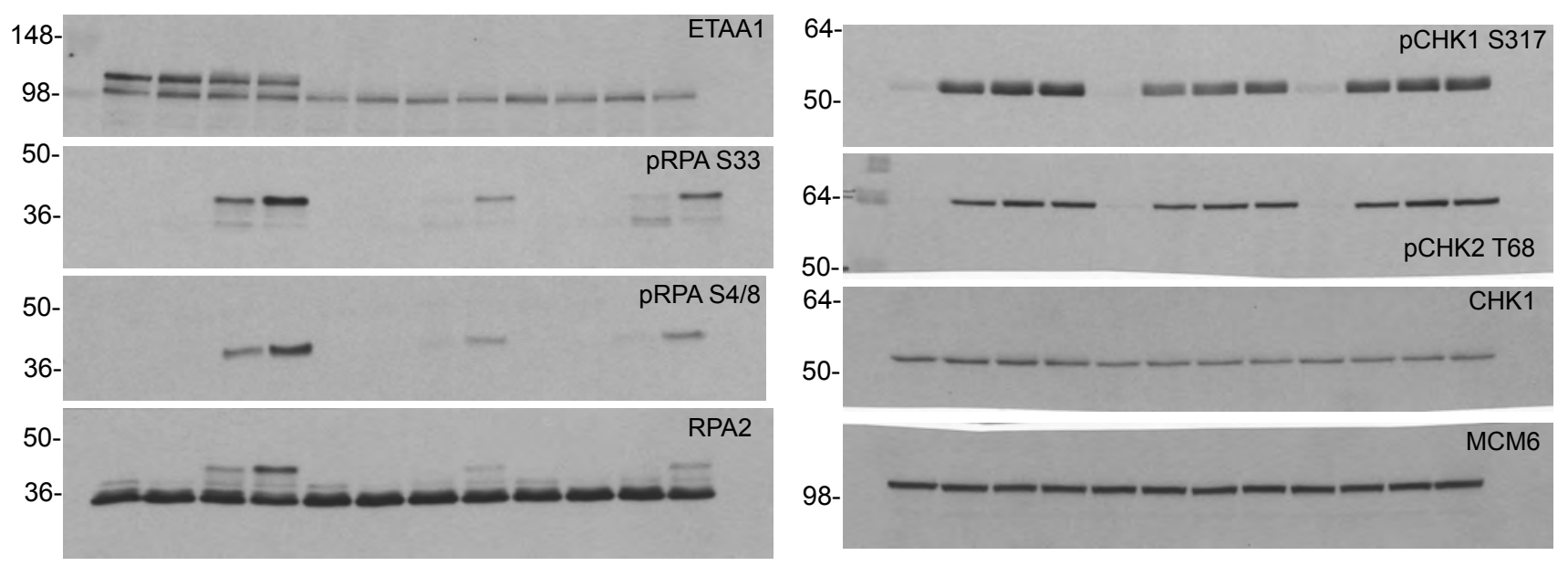




\section{Haahr et al. Figure S8}

Figure $4 b$

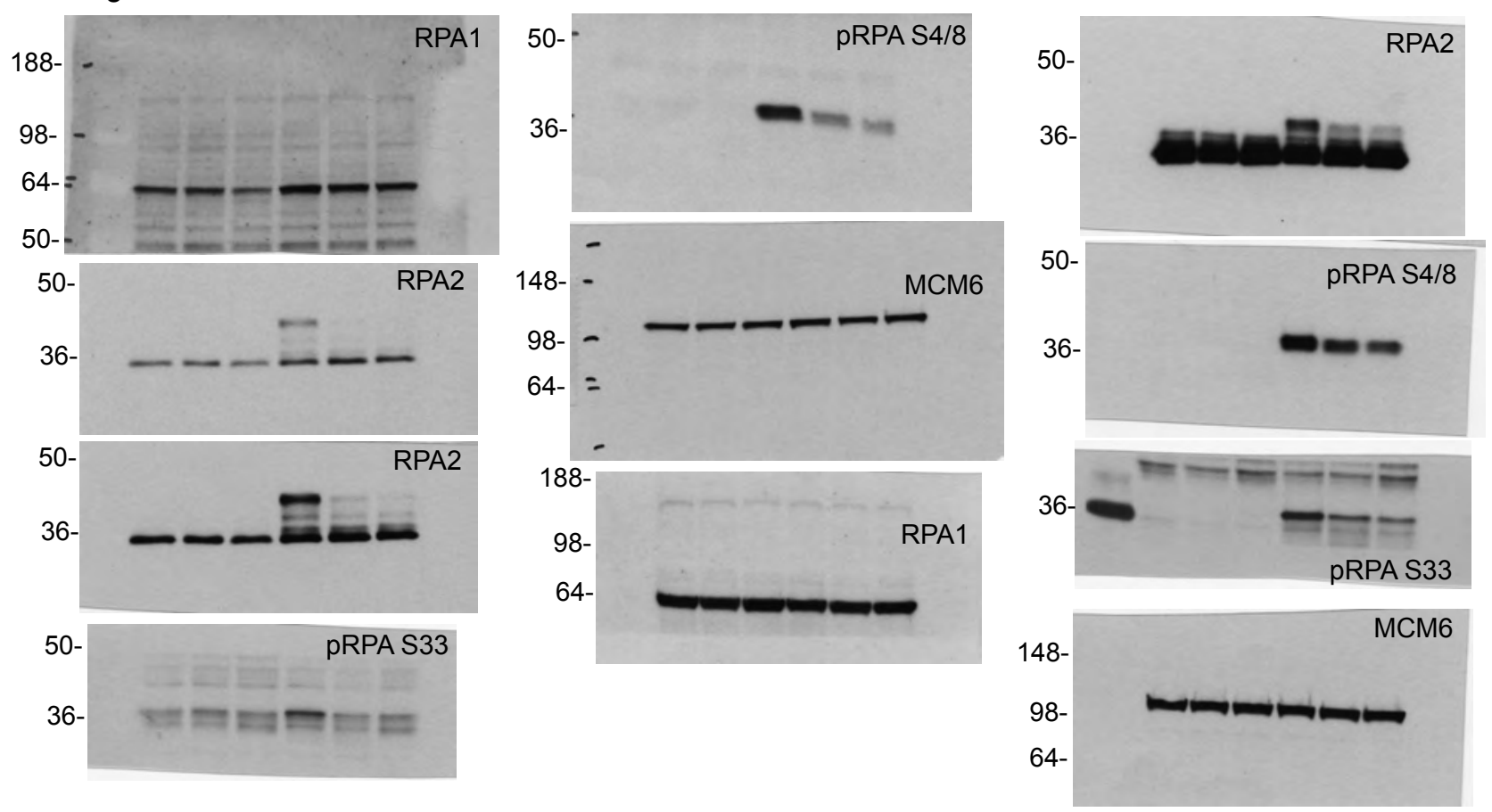

Figure 6b
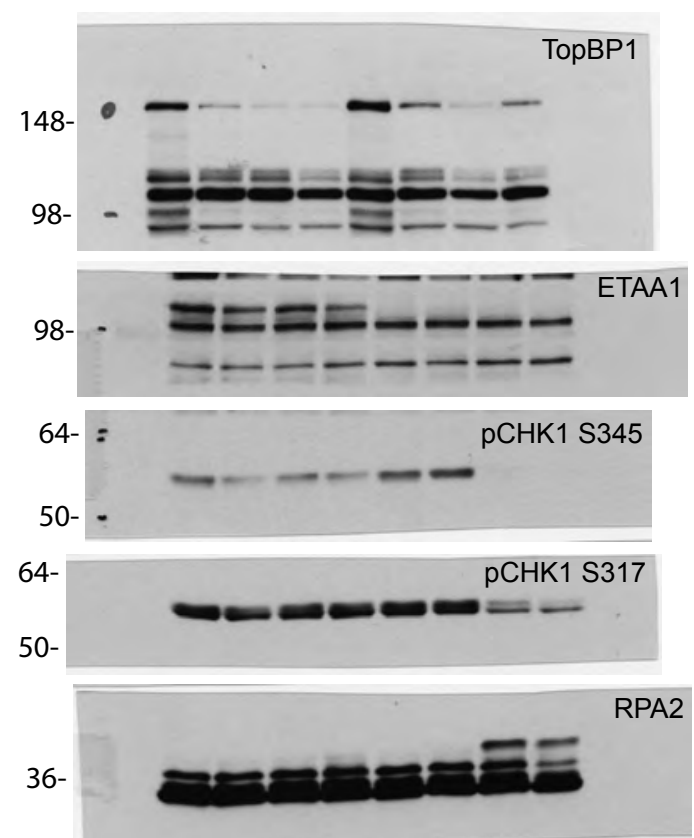
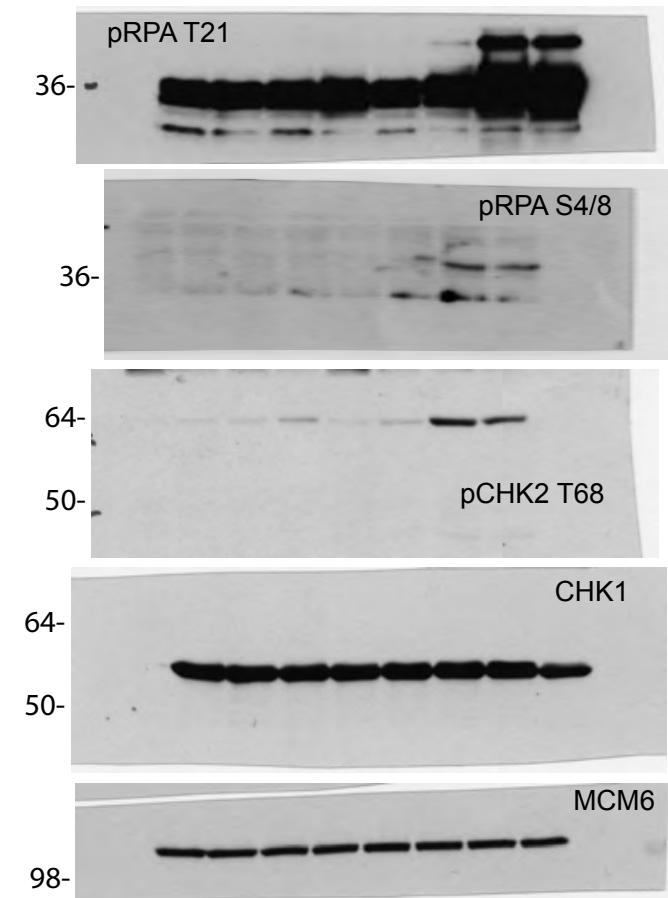

Figure $6 \mathrm{~g}$

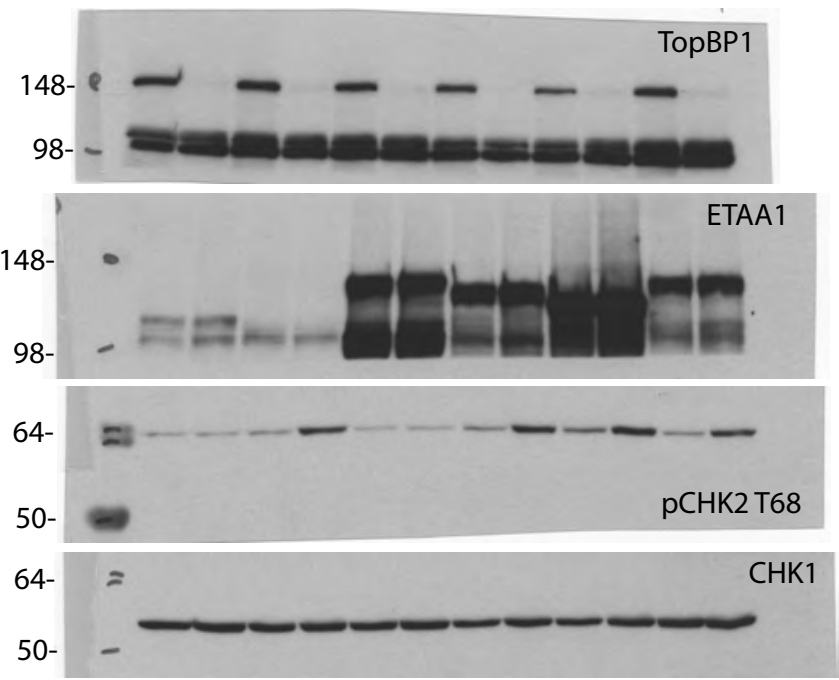

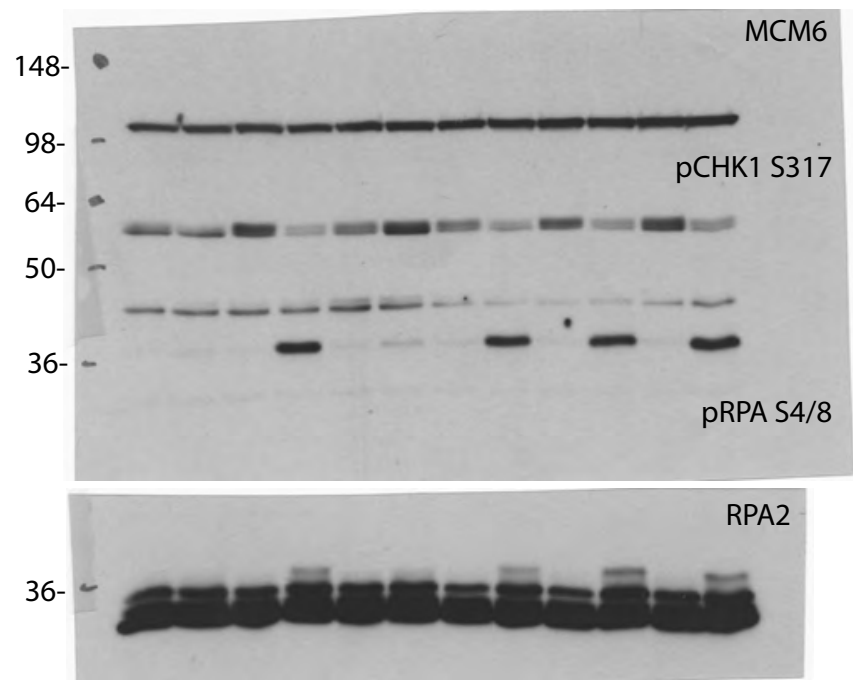




\section{Haahr et al. Figure S8}

Figure $7 a$
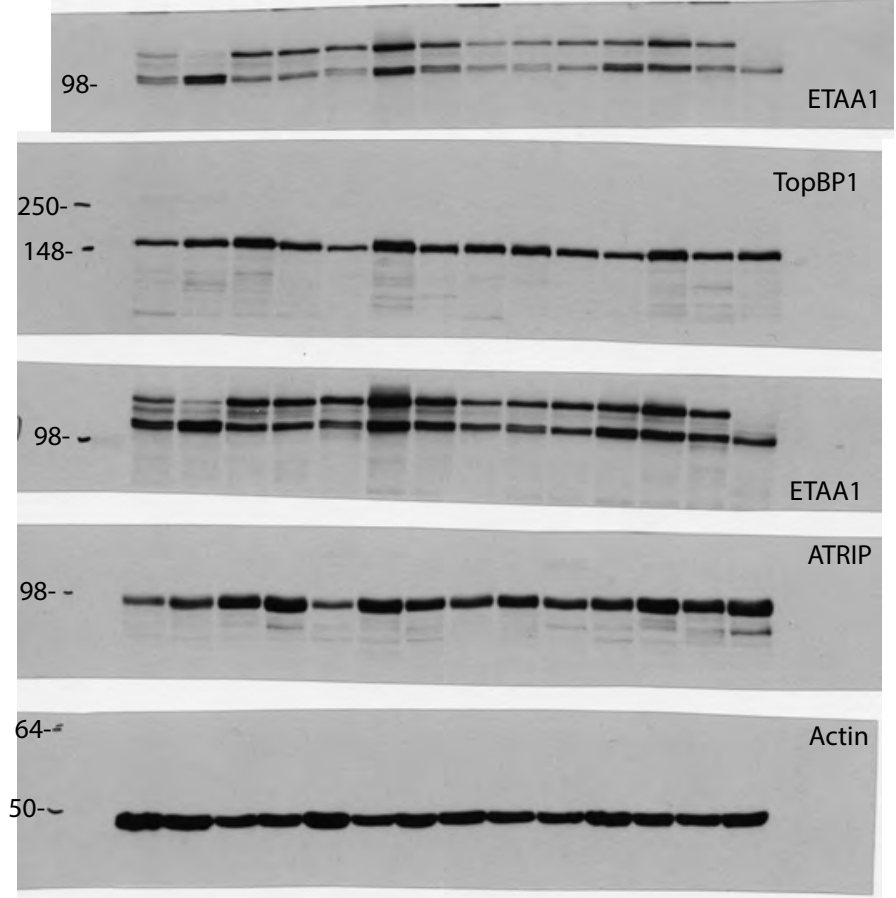

$250-$

148. - - - - - - - -
Figure 7b

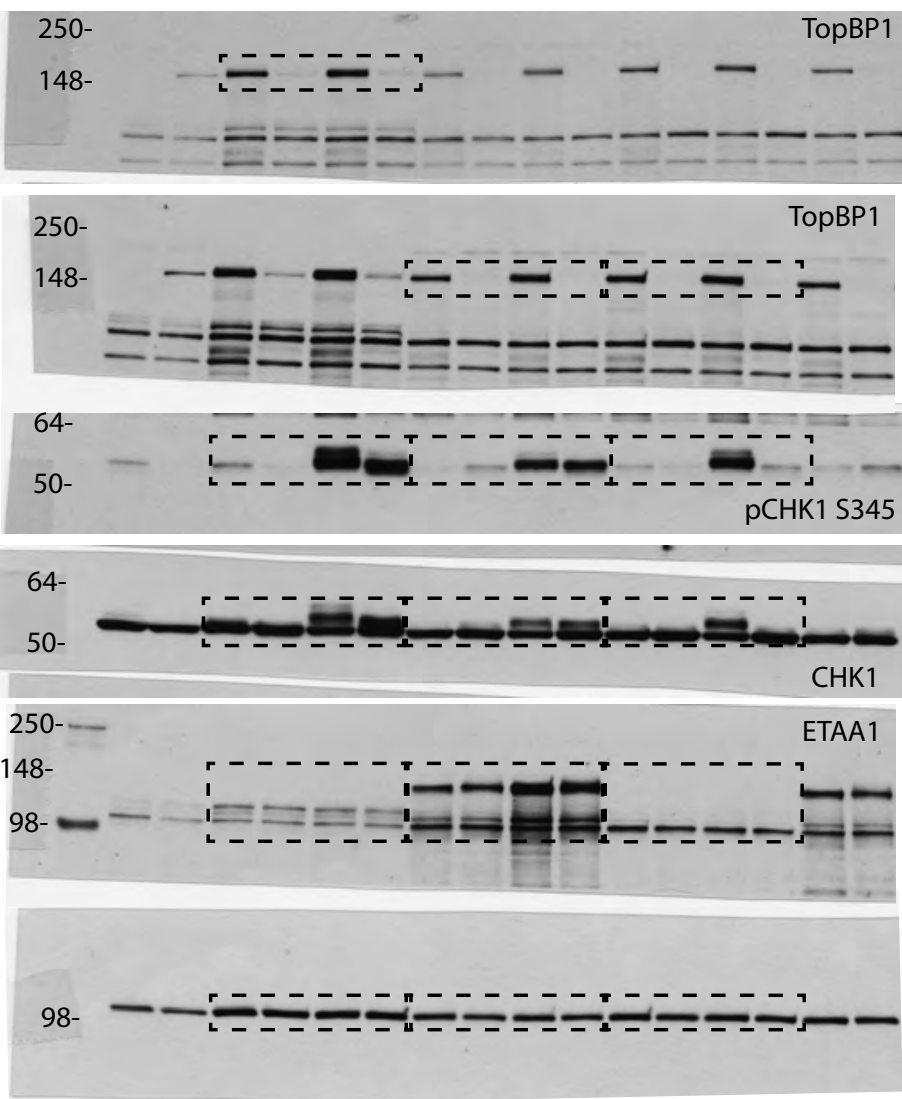

Figure S2a

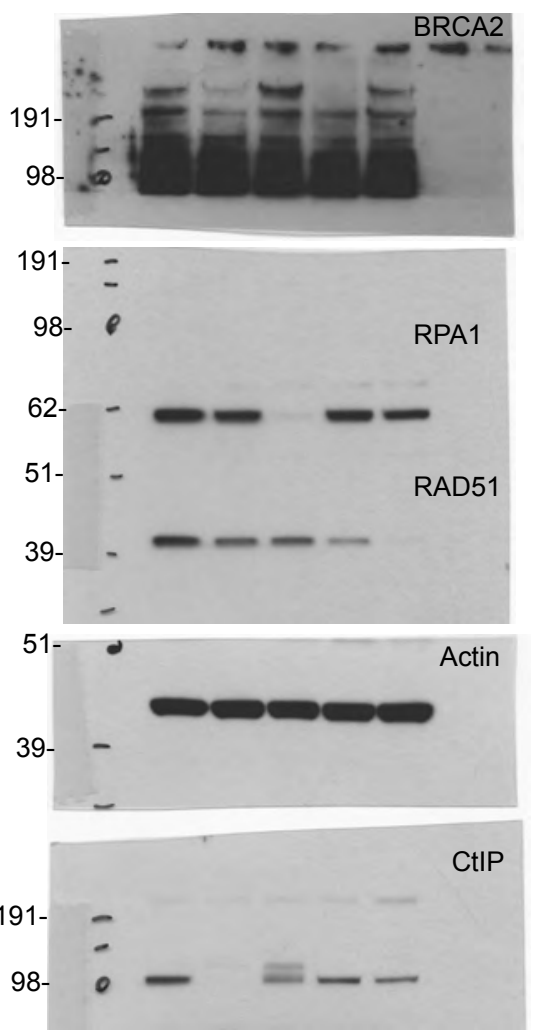

Figure S2e

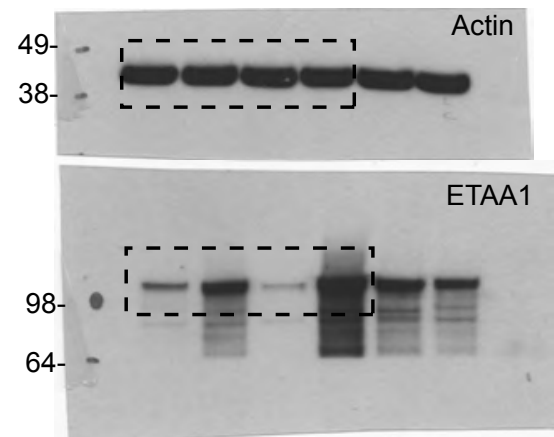

Figure S3g

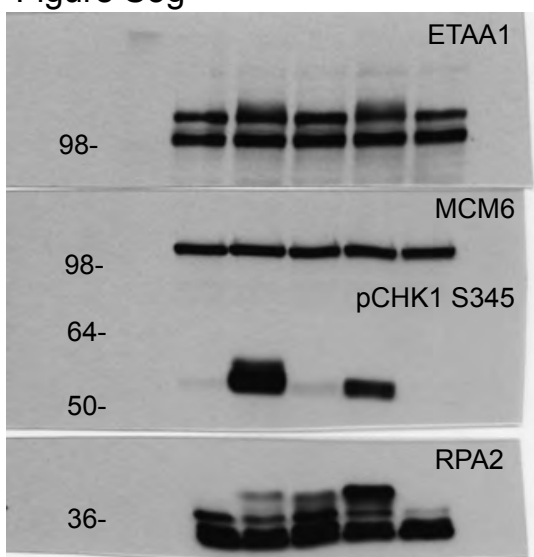

Figure S4c

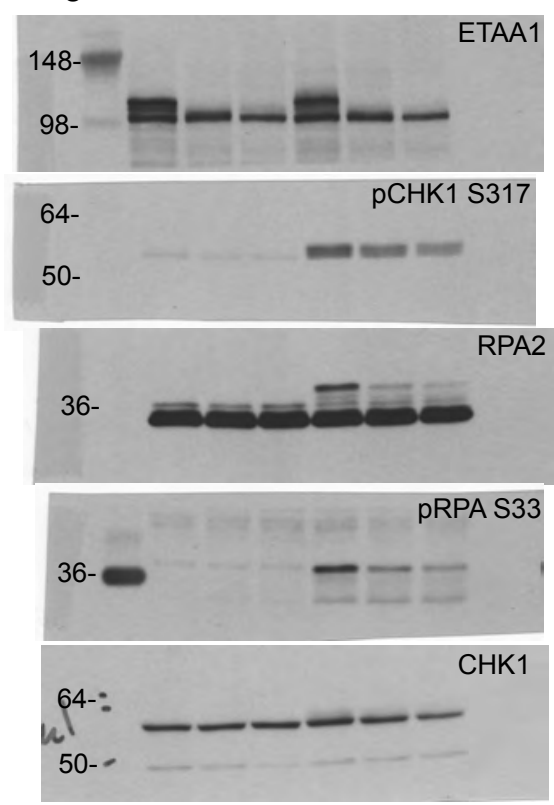

Figure S2b

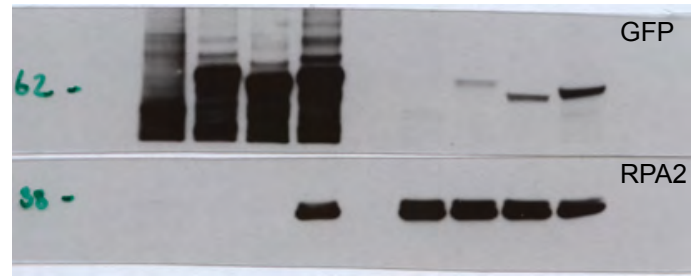

Figure S2f

191-




\section{Haahr et al. Figure S8}

Figure S4d

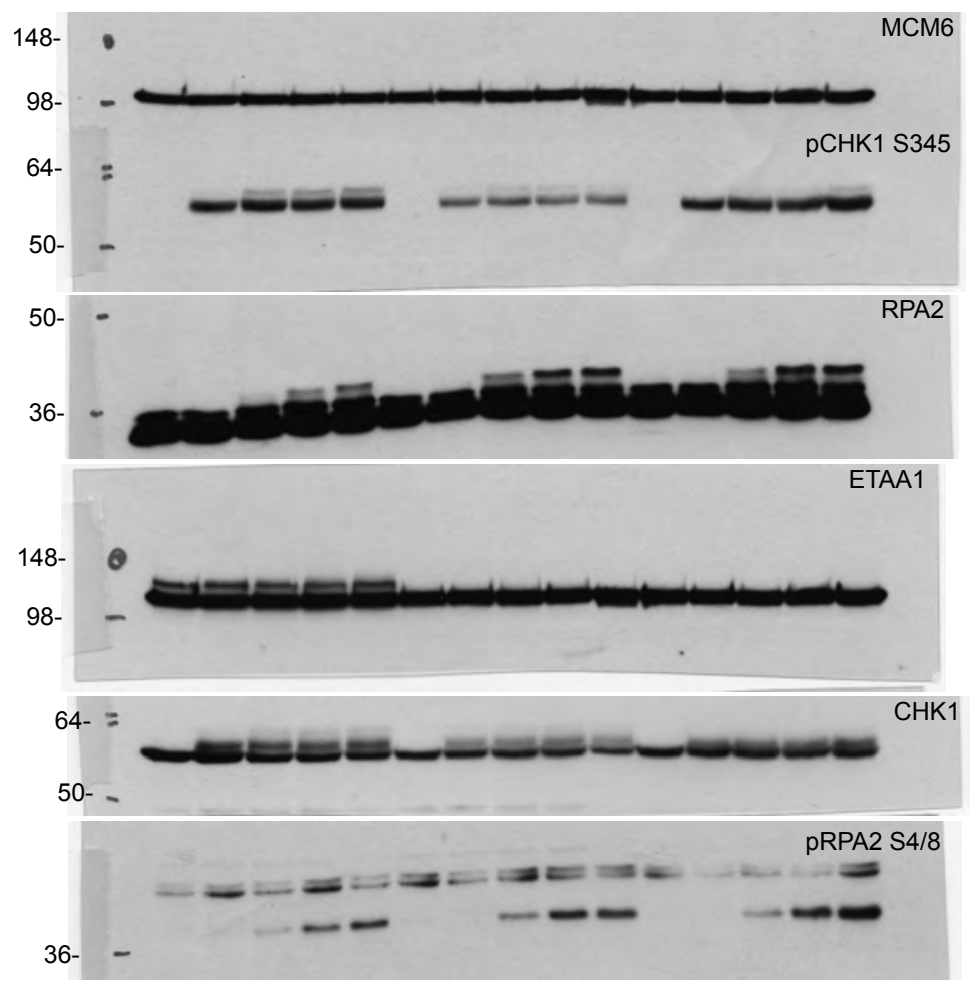

Figure S4i

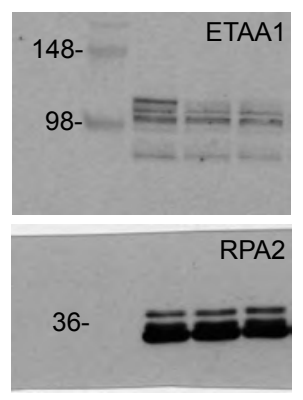

Figure S4k
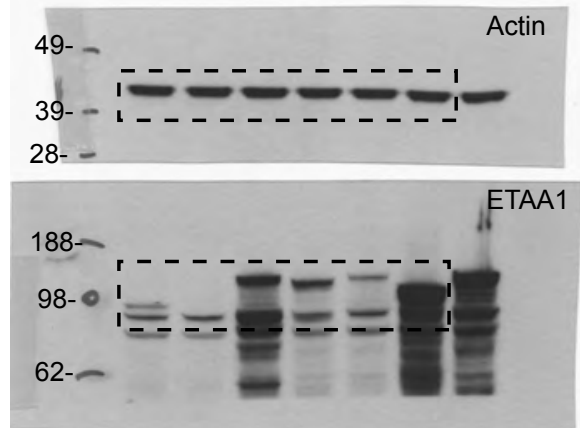

Figure S5d

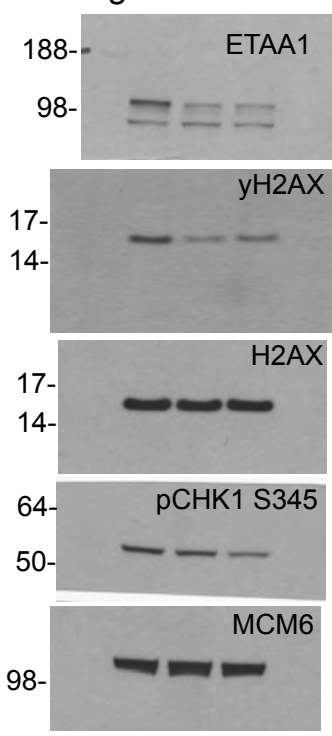

Figure S6c

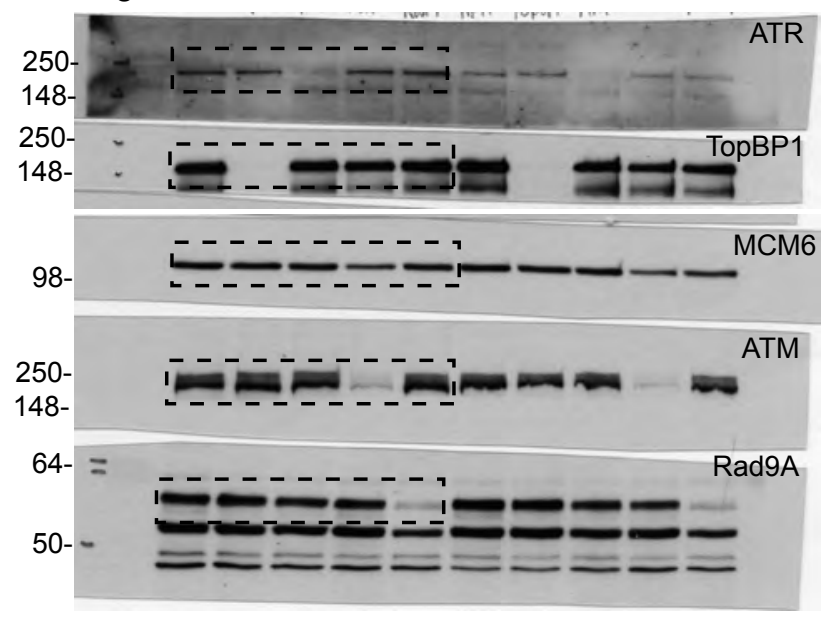

Figure S6f

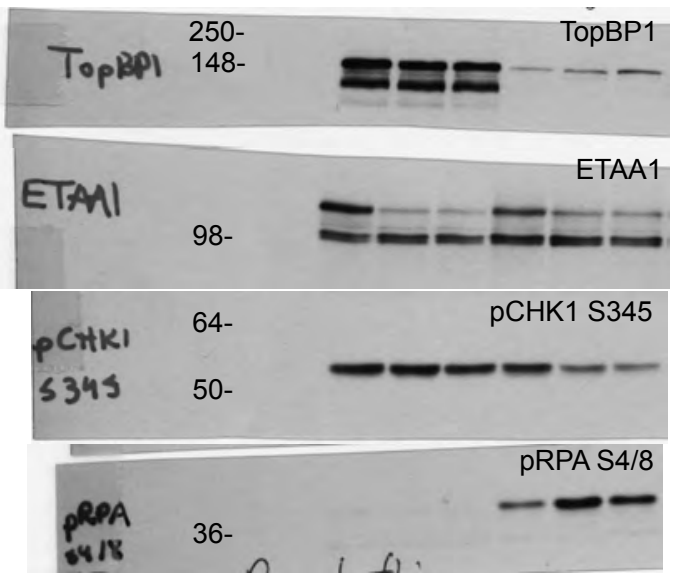

Figure S6e
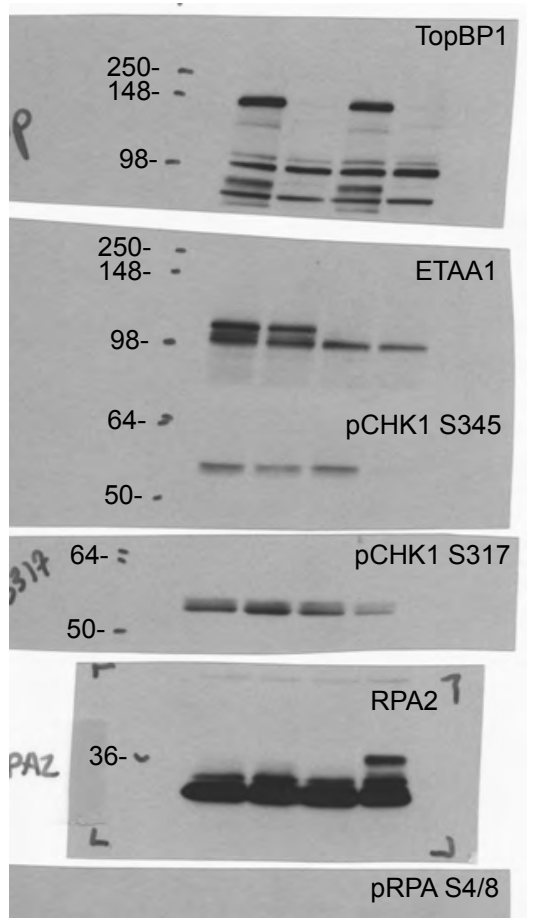

36--

MCM6

98-

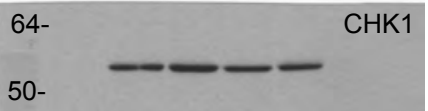




\section{Haahr et al. Figure S8}

Figure S6g

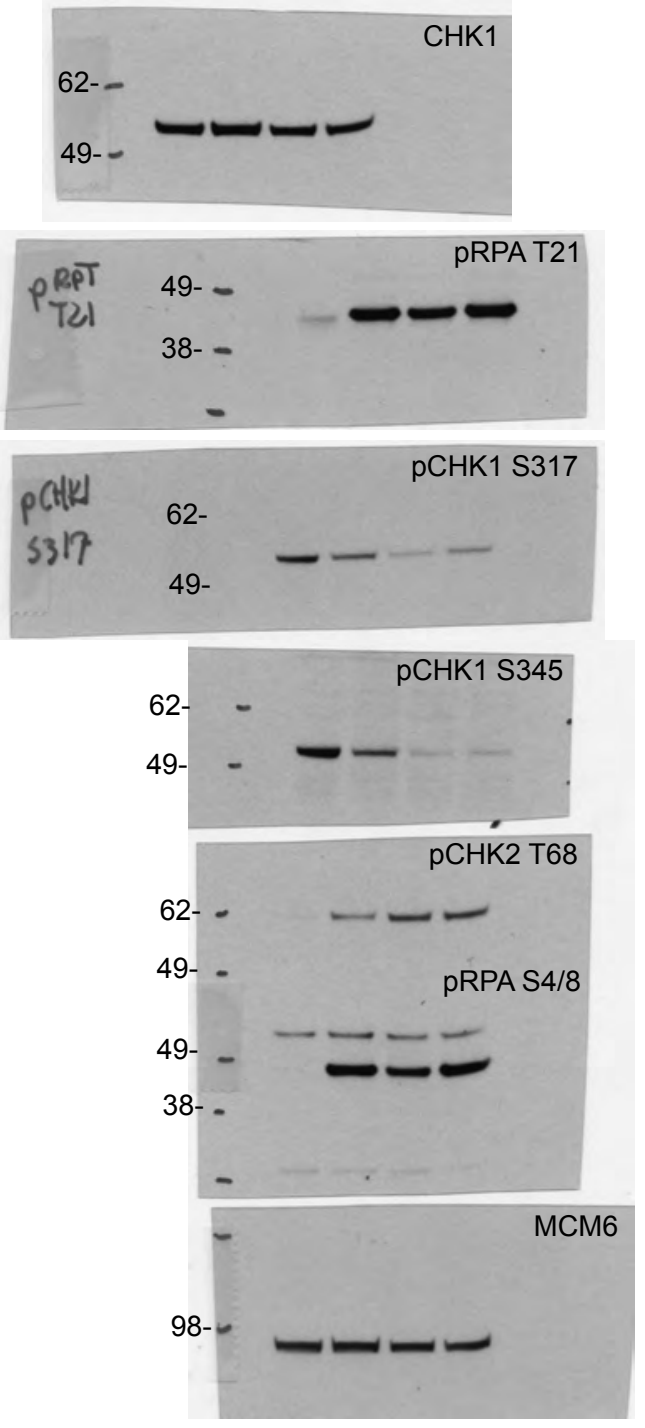

Figure S7c

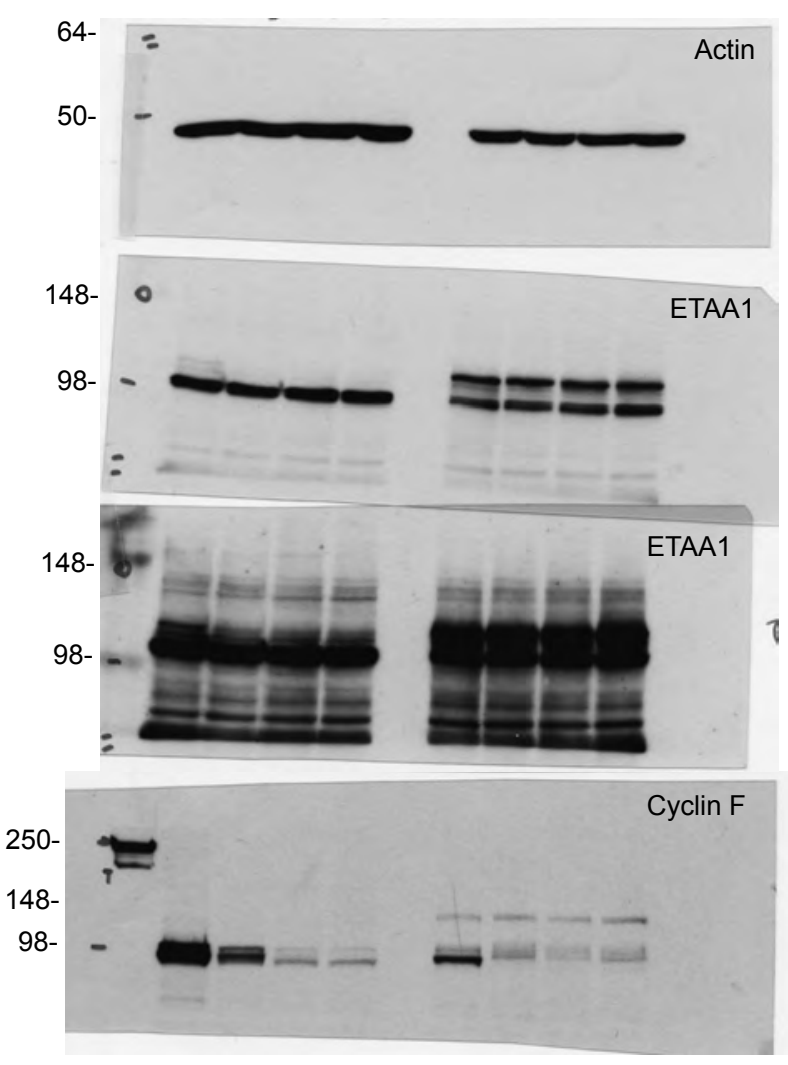

Figure S7f

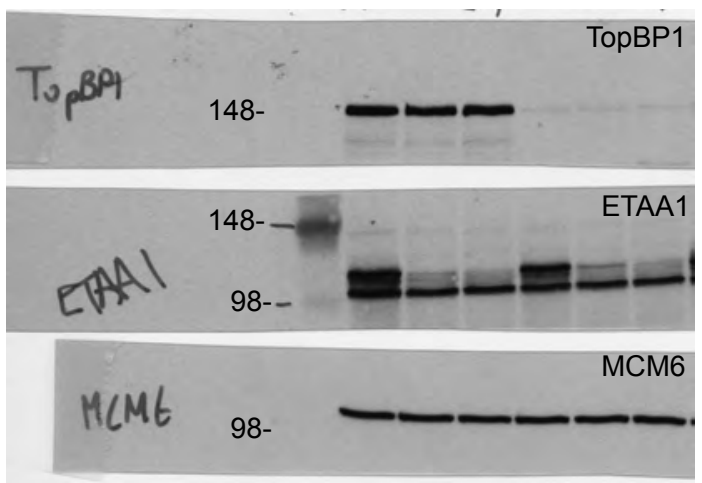

